Review

\title{
Long-term Trends of Organic Carbon Concentrations in Freshwaters: Strengths and Weaknesses of Existing Evidence
}

\author{
Montserrat Filella $^{1,2, *}$ and Juan Carlos Rodríguez-Murillo ${ }^{3}$ \\ 1 Institute F.-A. Forel, University of Geneva, Route de Suisse 10, Versoix CH-1290, Switzerland \\ 2 SCHEMA, Rue Principale 92, Rameldange L-6990, Luxembourg \\ 3 Museo Nacional de Ciencias Naturales, CSIC, Madrid E-28006, Spain; \\ E-Mail: jcmurillo@ccma.csic.es
}

* Author to whom correspondence should be addressed; E-Mail: montserrat.filella@unige.ch;

Tel.: +41-223-790-300; Fax: +41-223-790-329.

Received: 26 March 2014; in revised form: 25 April 2014 / Accepted: 4 May 2014 /

Published: 20 May 2014

\begin{abstract}
Many articles published in the last few years start with the assumption that the past decades have seen an increase in dissolved organic carbon (DOC) concentrations in the rivers and lakes of the Northern Hemisphere. This study analyses whether the existing evidence supports this claim. With this aim, we have collected published studies where long series of organic carbon concentrations (i.e., longer than 10 years) were analyzed for existing trends and have carefully evaluated the 63 articles found. Information has been collated in a comprehensive and comparable way, allowing readers to easily access it. The two main aspects considered in our analysis have been the analytical methods used and the data treatment methods applied. Both are sensitive issues because, on the one hand, the difficulties associated with correctly determining organic carbon concentrations in surface waters are well known, while, on the other, dealing with real environmental data (i.e., lack of normality, censoring, missing values, etc.) is an extremely intricate matter. Other issues such as data reporting and the geographical location of the systems studied are also discussed. In conclusion, it is clear that organic carbon concentrations have increased in some surface waters in the Northern Hemisphere since the 1990s. However, due to a lack of data in many parts of the world, it is not known whether this phenomenon is general and, more importantly, in the areas for which such data do exist, the reporting and methodological problems in the published studies prevent any conclusion on the existence of a general temporal behavior of organic carbon from being drawn.
\end{abstract}


Keywords: organic carbon; DOC; dissolved organic carbon; temporal trends; time-series analysis; freshwaters; lakes; rivers

\section{Introduction}

Inland waters (ponds, lakes, wetlands, streams, rivers and reservoirs) occupy only a small fraction of the Earth's surface but have a disproportionate effect on the global carbon cycle. A large amount of the carbon taken up by terrestrial system ends up in inland waters. The resulting riverine export of terrestrial organic matter to the oceans is a key link between terrestrial and marine parts of the global carbon cycle. While the amount of carbon transported is small compared with the massive fluxes between atmosphere and land and oceans, overall it accounts for about half of the net ecosystem production [1,2]. Moreover, inland waters do not act merely as passive "pipes" for carbon transport; rather they are active components of the carbon cycle because organic carbon (OC) in freshwater bodies can also be buried in sediments or mineralized and released back into the atmosphere as carbon dioxide.

Over the past decades, it has become increasingly accepted that dissolved organic carbon (DOC) concentrations have been increasing in rivers and lakes of the Northern Hemisphere. If confirmed, DOC increase may have significant impacts, not only on the global carbon cycle, but also on freshwater food chains, the quality of drinking water and trace element and organic micropollutant circulation and ecotoxicity. The root causes of this increase remain unclear. Although a few review articles on the subject have been published [3-6], they largely uncritically accept the universality of DOC increase, collate published results and list suggested causes. They very seldom address issues related to the methodology used, quality of the results, etc. This critical study is an attempt to clarify the situation by analyzing published increasing trends and, in particular, the reliability of the analytical and data treatment methods used and, finally, to evaluate to what extent they are not the result of a belief system generated by a so-called "repetition cascade" (i.e., repetition of claims) [7].

\section{Methods}

For literature searches we used the ISI Web of Science. Careful reading of published papers led to other references. Data considered in this study were restricted to studies published in peer-reviewed papers. Grey literature (i.e., documentary material that is not commercially published, typical examples being technical reports and conference proceedings) was not included. Because of this choice, some presumably interesting results such as those of Monteith and Evans [8], Skjelkvåle [9], Stoddard et al. [10], Gruau and co-workers on French rivers [11,12] or Zobrist et al. [13] are not included. Although one might argue that taking account of some grey literature might be worthwhile, the facts are that its reliability is always difficult to assess due to the absence of peer-review control and that it is often difficult to access. These reasons finally prevailed. Note, however, that in many cases these data are either totally or partially published later in refereed articles, cases in point being Freeman et al. [14] and Evans et al. [15,16], who refer to Monteith and Evans [8], and Eikebrokk et al. [17] and Skjelkvåle et al. [18], who refer to Skjelkvåle [9]. 
Articles where changes in OC concentration have been studied over short periods of time (e.g., seasonal studies) or extensive comparisons among freshwater bodies in different climatic zones (e.g., [19]) that do not contain long time-series data have been excluded from this study. Since time scales that are too short do not allow long-term trends to be reliably detected, studies based on less than $\approx 10$-year data series have not been included. On the other hand, studies where some surrogate parameter of OC (i.e., color, absorbance, chemical oxygen demand) was measured instead of OC itself have been included. Finally, articles discussing trends in OC fluxes but not in OC concentrations have not been considered.

\section{Results and Discussion}

Sixty-three articles containing long-term OC concentration series have been identified. A few more studies reiterating previously published data or results (and giving the initial publication as a reference) are cited but not considered in the set. Publication dates span from 1989 to 2012. The key information contained in these studies has been collated in a systematic form in three tables. Table 1 collects information about geographical location, system characteristics, period covered, sampling frequency and data sources. Table 2 shows methodological -both analytical and statistical- information. Table 3 contains the trend results and, in order to facilitate the reading, some key information already provided in Tables 1 and 2. A significant effort has been made to give all key information in a simple and comparable way but this has not always been possible due to the disparate way in which ancillary and methodological information is sometimes given in the original articles.

\subsection{Starting Considerations}

\subsubsection{Data Quality Traceability}

Most of the long-series data come from government surveys and sometimes the published articles do not give either the characteristics of the water systems or the analytical methods used in detail. Where references are given, they sometimes refer to the grey literature, always difficult to access and assess, or to previous articles where the information is not always found. Since, in particular in the case of DOC concentrations, values depend heavily on the analytical procedure applied (see corresponding section), the reliability of the data and of the conclusions reached becomes, in practice, difficult to assess when sampling and methodological information is missing.

A point worth mentioning is that not all studies contain independent data. Rather, sometimes previously processed data are totally or partially reused in later studies. Regrettably, this is not always made clear in the articles in question (e.g., Dillon and co-workers publications on Canadian lakes, Worrall's on UK freshwaters). In some cases, such as in the many studies published by Worrall and co-workers based on UK data, tracking how the data sets are inter-related from the information given in the articles becomes really tricky. Connections between data sets are mentioned in Table 1 when clearly stated or when deduced after careful reading of the article but we are aware that we have probably not spotted all existing links.

A further factor that makes evaluating studies' reliability more difficult is the fact that very often non-transformed original data are not shown, even not in graphical form. When shown, it is mentioned in Table 2. Other types of data representation (e.g., month or annual means) are also mentioned. 
Table 1. Published studies containing long-term organic carbon concentration temporal trends in freshwaters. System characteristics, sampling details and data sources.

\begin{tabular}{|c|c|c|c|c|c|c|c|c|c|}
\hline \multirow[t]{2}{*}{ Ref. } & \multicolumn{6}{|c|}{ System } & \multirow[t]{2}{*}{ Period } & \multirow{2}{*}{$\begin{array}{l}\text { Sampling } \\
\text { frequency }\end{array}$} & \multirow[t]{2}{*}{ Data source } \\
\hline & Type $^{\text {a }}$ & No. & Country & Details & General characteristics & $\begin{array}{l}\text { Acid rain } \\
\text { recovery? }^{b}\end{array}$ & & & \\
\hline [20] & river & 1 & Germany & $\begin{array}{l}\text { River Elbe, km: } 585-620 ; \\
\text { number of samples unknown } \\
\text { Location on a map }\end{array}$ & $\begin{array}{l}\text { Freshwater tidal zone; } \\
\text { pre-oxygen minimum zone; } \\
\text { salinity }<1\end{array}$ & No & $1985-2007$ & $\begin{array}{l}\text { 1985-1993: almost monthly } \\
\text { 1994-2007: February, } \\
\text { May-August, November }\end{array}$ & ARGE Elbe \\
\hline [21] & lakes & 30 & $\begin{array}{l}\text { Quebec, } \\
\text { Canada }\end{array}$ & $\begin{array}{l}\text { Located } \mathrm{N} \text { of the St Lawrence } \\
\text { River between Ottawa and } \\
\text { Saguenay Rivers in Quebec } \\
\text { Location on a map }\end{array}$ & $\begin{array}{l}\text { Lake surface areas: } 0.061-2.02 \mathrm{~km}^{2} \\
\text { Max depth: } 3-38 \mathrm{~m} \\
\text { Water retention time: } 0.1-9 \mathrm{y} \\
\text { (median: } 1.8 \text { ) } \\
\text { Catchment areas: } 0.42-6.96 \mathrm{~km}^{2}\end{array}$ & Yes & 1989-2006 & $\begin{array}{l}\text { Twice a year in } \\
\text { spring and fall }\end{array}$ & $\begin{array}{l}\text { Acid Rain Program of } \\
\text { Environment Canada }\end{array}$ \\
\hline [22] & stream & $\begin{array}{l}\text { not } \\
\text { clear }\end{array}$ & $\begin{array}{l}\text { Wales, } \\
\text { United } \\
\text { Kingdom }\end{array}$ & $\begin{array}{l}\text { Upper Hafren catchment, } \\
\text { subcatchment of the Upper River } \\
\text { Severn (Plynlimon) } \\
\text { Location on a map }\end{array}$ & Catchment area: $1.17 \mathrm{~km}^{2}$ & NM & 1990-2010 & Weekly & $\begin{array}{l}\text { Centre for Ecology and } \\
\text { Hydrology }(\mathrm{CEH}) \\
\text { Probably some data } \\
\text { already included in } \\
{[23]}\end{array}$ \\
\hline [24] & stream & 1 & $\begin{array}{l}\text { Ontario, } \\
\text { Canada }\end{array}$ & $\begin{array}{l}\text { Plastic Lake catchment } \\
\text { Location on a map }\end{array}$ & Small ephemeral stream & Yes & $\begin{array}{l}\text { 1987-1994, } \\
1999-2009\end{array}$ & $\begin{array}{l}\text { Not given; probably } \\
\text { information in [25] }\end{array}$ & $\begin{array}{l}\text { Monitoring program, } \\
\text { Ontario Ministry of } \\
\text { Environment }\end{array}$ \\
\hline [26] & rivers & 11 & Estonia & $\begin{array}{l}\text { Large: Narva, Suur Emajõgi, } \\
\text { Pärnu; small in N Estonia: Kasari, } \\
\text { Vihterpalu, Keila, Vääna, Pudisoo, } \\
\text { Valgejõgi; small in S Estonia: } \\
\text { Väike Emajõgi, Võhandu } \\
\text { Location on a map }\end{array}$ & $\begin{array}{l}\text { Total catchment area: } \\
57,619 \mathrm{~km}^{2}\end{array}$ & NM & $\begin{array}{l}\text { TOC: } \\
\text { 1998-2007 } \\
\text { COD: } \\
\text { 1992-2007 }\end{array}$ & 6-12 times a year & $\begin{array}{l}\text { Estonian national } \\
\text { environmental } \\
\text { monitoring programme }\end{array}$ \\
\hline
\end{tabular}


Table 1. Cont

\begin{tabular}{|c|c|c|c|c|c|c|c|c|c|}
\hline \multirow[t]{2}{*}{ Ref. } & \multicolumn{6}{|c|}{ System } & \multirow[t]{2}{*}{ Period } & \multirow{2}{*}{$\begin{array}{l}\text { Sampling } \\
\text { frequency }\end{array}$} & \multirow[t]{2}{*}{ Data source } \\
\hline & Type $^{\text {a }}$ & No. & Country & Details & General characteristics & $\begin{array}{l}\text { Acid rain } \\
\text { recovery? }^{b}\end{array}$ & & & \\
\hline [27] & lakes & 91 & Canada & $\begin{array}{l}\text { Atlantic Provinces: Newfoundland } \\
\text { (NF) (14 sites), southwestern Nova } \\
\text { Scotia (WNS) (45), eastern Nova } \\
\text { Scotia (ENS) (23), southwestern } \\
\text { New Brunswick (NB) (13) } \\
\text { No list of sites given } \\
\text { Approximate location on a map }\end{array}$ & - & Yes & $\begin{array}{l}\text { NF, WNS: } \\
\text { 1983-2007 } \\
\text { ENS: } \\
\text { 1990-2007 } \\
\text { NB: } \\
\text { 2000-2007 }\end{array}$ & $\begin{array}{l}\text { Semi-annually, during } \\
\text { spring and fall overturn } \\
\text { from May to October }\end{array}$ & $\begin{array}{l}\text { Environment Canada } \\
\text { monitoring at four } \\
\text { Canadian Air and } \\
\text { Precipitation } \\
\text { Monitoring Network } \\
(\mathrm{CAPMoN})\end{array}$ \\
\hline [28] & lake & 1 & Switzerland & Lake Maggiore & $\begin{array}{l}\text { Subalpine lake, recovered from } \\
\text { eutrophic period in the late 1970's } \\
\text { Lake surface area: } 212 \mathrm{~km}^{2} \\
\text { Max depth: } 372 \mathrm{~m}\end{array}$ & No & $1980-2007$ & $\begin{array}{l}\text { Monthly: } \\
\text { Nov, Dec, Jan, Feb; } \\
\text { fortnightly: } \\
\text { other months }\end{array}$ & - \\
\hline [29] & stream & 6 & $\begin{array}{l}\text { United } \\
\text { Kingdom }\end{array}$ & $\begin{array}{l}\text { South Pennines: Trout Beck } \\
\text { (Moor House) } \\
\text { South Pennines: Lower Laithe, } \\
\text { Keighley Moor, Agden, } \\
\text { Broomhead, Langsett } \\
\text { Location on a map }\end{array}$ & Peat-rich catchments & Yes & $\begin{array}{l}\text { T’ Beck: } \\
\text { 1993-2006 } \\
\text { L' Laithe: } \\
\text { 1994-2006 } \\
\text { K' Moor: } \\
\text { 1979-2006 } \\
\text { Agden, } \\
\text { Broomhead, } \\
\text { Langsett: } \\
\text { 1961-2006 }\end{array}$ & $\begin{array}{l}\text { T' Beck: weakly } \\
\text { Others: not clear }\end{array}$ & $\begin{array}{l}\text { T' Beck: Environmental } \\
\text { Change Network }(\mathrm{ECN}) \\
\text { Data from T' Beck } \\
\text { already published in } \\
{[\mathbf{3 0 , 3 1 ]}}\end{array}$ \\
\hline [32] & stream & 1 & USA & Bear Brook watershed, Maine & $\begin{array}{l}\text { Low-alkalinity headwater } \\
\text { stream }\end{array}$ & Yes & $\begin{array}{l}1988-1989, \\
1990-1995, \\
1996-2006\end{array}$ & Weekly & - \\
\hline
\end{tabular}


Table 1. Cont

\begin{tabular}{|c|c|c|c|c|c|c|c|c|c|}
\hline \multirow[t]{2}{*}{ Ref. } & \multicolumn{6}{|c|}{ System } & \multirow[t]{2}{*}{ Period } & \multirow{2}{*}{$\begin{array}{l}\text { Sampling } \\
\text { frequency }\end{array}$} & \multirow[t]{2}{*}{ Data source } \\
\hline & Type $^{\text {a }}$ & No. & Country & Details & General characteristics & $\begin{array}{l}\text { Acid rain } \\
\text { recovery }^{b}\end{array}$ & & & \\
\hline [33] & $\begin{array}{l}\text { moorland } \\
\text { pools }\end{array}$ & 4 & $\begin{array}{l}\text { Nether- } \\
\text { lands }\end{array}$ & $\begin{array}{l}\text { Achterste Goorven (AG), } \\
\text { Groot Huisven, } \\
\text { MiddelsteWolfsputven, Schaapsven } \\
\text { Location in a map }\end{array}$ & No characteristics given & Yes & 1978-2006 & $\begin{array}{l}\text { AG: } 4 \text { times/year } \\
\text { (every season) } \\
\text { The rest: once every } 4 \text { years }\end{array}$ & \\
\hline [34] & lakes & 55 & Canada & $\begin{array}{l}\text { Ontario: Dorset (8), } \\
\text { ELA (4), Turkey (TLW) (5); } \\
\text { Nova Scotia: Kejimkujik (26), } \\
\text { Yarmouth (11) } \\
\text { No list, approximate location on a map }\end{array}$ & $\begin{array}{l}\text { Summary of lake } \\
\text { characteristics in the } \\
\text { article }\end{array}$ & Yes & 1981-2003 & $\begin{array}{l}\text { Ontario: } \\
5-24 \text { times a year, from } \\
\text { May to October } \\
\text { Nova Scotia: } \\
1 \text { spring, } 1 \text { autumn } \\
\end{array}$ & $\begin{array}{l}\text { Different sources } \\
\text { Includes, at least, [35] } \\
\text { data }\end{array}$ \\
\hline [36] & streams & 2 & $\begin{array}{l}\text { Czech } \\
\text { Republic }\end{array}$ & $\begin{array}{l}\text { Lysina, Pluhuv Bor } \\
\text { No map }\end{array}$ & $\begin{array}{l}\text { Lysina: acidic, catchment } \\
0.273 \mathrm{~km}^{2} \\
\text { Pluhuv Bor: } \\
\text { well-buffered catchment } \\
0.216 \mathrm{~km}^{2}\end{array}$ & Yes & 1993-2007 & Weekly & - \\
\hline$[37]$ & $\begin{array}{l}\text { reservoirs } \\
\text { streams }\end{array}$ & $\begin{array}{l}11 \\
4\end{array}$ & $\begin{array}{l}\text { Czech } \\
\text { Republic }\end{array}$ & $\begin{array}{l}\text { Ore Mountains (Krušné hory) } \\
\text { List of names, location on a map }\end{array}$ & Catchments: 8-74 km² & Yes & $\begin{array}{l}\text { reservoirs: } \\
\text { 1969-2006 } \\
\text { streams: } \\
(1969,1974, \\
1983)-2006 \\
\end{array}$ & Median sampling: 34 days & $\begin{array}{l}\text { Ohre River and Labe } \\
\text { River Authorities }\end{array}$ \\
\hline [38] & streams & 8 & Finland & $\begin{array}{l}\text { Forested headwater catchments, } \\
\text { eastern Finland: Murtopuro, } \\
\text { Liuhapuro, Suoputo, Kivipuro, } \\
\text { Välipuro, Porkkavaara, } \\
\text { Kangaslampi, Korsukorpi } \\
\text { No map }\end{array}$ & $\begin{array}{l}\text { Catchments: } \\
0.29-4.94 \mathrm{~km}^{2}\end{array}$ & Yes & $\begin{array}{l}\text { 2: 1979-2006 } \\
\text { 3: 1979-1982, } \\
\text { 1996-2005 } \\
\text { 3: 1992-2006 }\end{array}$ & $\begin{array}{l}\text { Variable, described in } \\
\text { the article }\end{array}$ & - \\
\hline
\end{tabular}


Table 1. Cont

\begin{tabular}{|c|c|c|c|c|c|c|c|c|c|}
\hline \multirow[t]{2}{*}{ Ref. } & \multicolumn{6}{|c|}{ System } & \multirow[t]{2}{*}{ Period } & \multirow{2}{*}{$\begin{array}{l}\text { Sampling } \\
\text { frequency }\end{array}$} & \multirow[t]{2}{*}{ Data source } \\
\hline & Type $^{\text {a }}$ & No. & Country & Details & General characteristics & $\begin{array}{l}\text { Acid rain } \\
\text { recovery? }^{b}\end{array}$ & & & \\
\hline [39] & streams & 3 & Canada & $\begin{array}{l}\text { Streams: Mersey, Moose Pit Brook, } \\
\text { Pine Marten Brook (Southwestern } \\
\text { Nova Scotia) } \\
\text { Location on a map }\end{array}$ & $\begin{array}{l}\text { Catchments: } 297 \mathrm{~km}^{2} \\
\text { (Mersey), } \\
17 \mathrm{~km}^{2} \text { (Moose Pit Brook), } \\
1.3 \mathrm{~km}^{2} \text { (Pine Marten Brook) }\end{array}$ & Yes & $\begin{array}{l}\text { Mersey: } \\
\text { 1980-2005 } \\
\text { Moose Pit: } \\
\text { 1983-2005 } \\
\text { Pine Marten: } \\
\text { 1991-2005 }\end{array}$ & Weekly & - \\
\hline [40] & streams & 6 & $\begin{array}{l}\text { Scotland, } \\
\text { United } \\
\text { Kingdom }\end{array}$ & $\begin{array}{l}\text { Loch Ard ( } 3 \text { sites: Burns 2, 10, 11), } \\
\text { Allt a'Mharcaidh, Sourhope } \\
\text { (Alderhope and Rowantree Bruns) } \\
\text { Location on a map }\end{array}$ & $\begin{array}{l}\text { Catchments: } \\
0.44-10 \mathrm{~km}^{2}\end{array}$ & Yes & $\begin{array}{l}\text { Burn 2: } \\
\text { 1989-2002 } \\
\text { Burns 10, 11: } \\
\text { 1988-2003 } \\
\text { Allt a'Mharcaidh: } \\
\text { 1987-2002 } \\
\text { Sourhope: } \\
\text { 1995-2006 }\end{array}$ & At least fortnightly & $\begin{array}{l}\text { UK Acid Waters } \\
\text { Monitoring Network } \\
(\text { AWMN) and } \\
\text { Environmental Change } \\
\text { Network (ECN) } \\
\text { Loch Ard data in } \\
\text { [41,42] }\end{array}$ \\
\hline [43] & streams & 7 & $\begin{array}{l}\text { Ontario, } \\
\text { Canada }\end{array}$ & $\begin{array}{l}\text { Harp Lake (6 catchments), } \\
\text { Plastic Lake ( } 1 \text { catchment) } \\
\text { No map }\end{array}$ & $\begin{array}{l}\text { Headwater catchments: } \\
0.097-1.905 \mathrm{~km}^{2}\end{array}$ & Yes & 1980-2002 & $\begin{array}{l}\text { Weekly or fortnightly, more } \\
\text { frequently during periods of } \\
\text { high discharge } \\
\text { Total: } 1530 \text { (PC), } 2200 \\
\text { (HP) }\end{array}$ & $\begin{array}{l}\text { Ontario Ministry of } \\
\text { Environment Dorset } \\
\text { Environmental Science } \\
\text { Centre (DESC) }\end{array}$ \\
\hline [44] & streams & 7 & $\begin{array}{l}\text { Ontario, } \\
\text { Canada }\end{array}$ & $\begin{array}{l}\text { Same data as [43] } \\
\text { No map }\end{array}$ & Same data as [43] & Yes & $1980-2002$ & See [43] & Same data as [43] \\
\hline [45] & stream & 1 & $\begin{array}{l}\text { Ontario, } \\
\text { Canada }\end{array}$ & $\begin{array}{l}\text { Plastic Lake (PC1) } \\
\text { No map }\end{array}$ & $\begin{array}{l}\text { Wetland dominated } \\
\text { catchment: } \\
0.234 \mathrm{~km}^{2}\end{array}$ & Yes & $1980-2001$ & See $[43]$ & $\begin{array}{l}\text { This catchment is } \\
\text { included in }[43]\end{array}$ \\
\hline
\end{tabular}


Table 1. Cont

\begin{tabular}{|c|c|c|c|c|c|c|c|c|c|}
\hline \multirow[t]{2}{*}{ Ref. } & \multicolumn{6}{|c|}{ System } & \multirow[t]{2}{*}{ Period } & \multirow{2}{*}{$\begin{array}{l}\text { Sampling } \\
\text { frequency }\end{array}$} & \multirow[t]{2}{*}{ Data source } \\
\hline & Type $^{\text {a }}$ & No. & Country & Details & General characteristics & $\begin{array}{l}\text { Acid rain } \\
\text { recovery? }^{\text {b }}\end{array}$ & & & \\
\hline [46] & rivers & 21 & Sweden & No list, no map & Catchments: $210-26,800 \mathrm{~km}^{2}$ & Yes & $\begin{array}{l}\text { TOC: } 1987- \\
2004 \\
\text { A, COD: } 1970- \\
2004\end{array}$ & $\begin{array}{l}\text { TOC: not given } \\
\text { A, COD: monthly }\end{array}$ & $\begin{array}{l}\text { Rivers included in } \\
\text { national or regional } \\
\text { monitoring programs (not } \\
\text { detailed) }\end{array}$ \\
\hline [47] & $\begin{array}{l}\text { lake } \\
\text { stream }\end{array}$ & $\begin{array}{l}1 \\
1\end{array}$ & Finland & $\begin{array}{l}\text { Valkea-Kotinen, lake and } \\
\text { catchment outflow } \\
\text { Location on a map }\end{array}$ & $\begin{array}{l}\text { Headwater catchment: } 0.30 \mathrm{~km}^{2} \\
\text { Mean depth: } 3 \mathrm{~m} \\
\text { Volumen: } 77,000 \mathrm{~m}^{3}\end{array}$ & Yes & 1990-2003 & Not given & $\begin{array}{l}\text { Lake already studied in } \\
{[48], \text { same results }}\end{array}$ \\
\hline [49] & lakes & 12 & $\begin{array}{l}\text { Ontario, } \\
\text { Canada }\end{array}$ & $\begin{array}{l}\text { Boreal Shield lakes: } 5 \text { near } \\
\text { Sudbury } \\
\text { (very acidified), } 7 \text { near Dorset } \\
\text { (less affected) } \\
\text { Location on a map }\end{array}$ & $\begin{array}{l}\text { Lake area: } 0.058-0.936 \mathrm{~km}^{2} \\
\text { Max depth: } 8.0-38.0 \mathrm{~m}\end{array}$ & Yes & $\begin{array}{l}\text { Sudbury: (1981, } \\
\text { 1982, 1987)- } \\
2003 \\
\text { Dorset: 1978/9- } \\
2003\end{array}$ & $\begin{array}{l}\text { Monthly or more } \\
\text { frequently during ice- } \\
\text { free season }\end{array}$ & - \\
\hline$[50]$ & river & 1 & Finland & $\begin{array}{l}\text { Simojoki river, Finnish Lapland } \\
\text { Location on a map }\end{array}$ & Catchment: $3160 \mathrm{~km}^{2}$ & Yes & $1962-2005$ & $\begin{array}{l}\text { 1962-1981: } 4 \\
\text { samples per year } \\
\text { 1982-2005: 10-18 } \\
\text { samples per year }\end{array}$ & $\begin{array}{l}\text { Regional environment } \\
\text { center }\end{array}$ \\
\hline [51] & $\begin{array}{l}\text { lakes } \\
\text { and } \\
\text { streams }\end{array}$ & $\begin{array}{l}522(6 \\
\text { regions })\end{array}$ & $\begin{array}{l}\text { North } \\
\text { America } \\
\text { and } \\
\text { northern } \\
\text { Europe }\end{array}$ & No list, incomplete map & Remote systems & Yes & $1990-2004$ & Not given & $\begin{array}{l}\text { Data collated from several } \\
\text { regional and national } \\
\text { monitoring initiatives on } \\
\text { acid-sensitive terrain } \\
\text { Probably some data } \\
\text { already considered in } \\
\text { other studies }\end{array}$ \\
\hline [41] & streams & 2 & $\begin{array}{l}\text { Scotland, } \\
\text { United } \\
\text { Kingdom }\end{array}$ & $\begin{array}{l}\text { Loch Ard: Burn } 10\left(0.9 \mathrm{~km}^{2}\right) \\
\text { and Burn } 11\left(1.4 \mathrm{~km}^{2}\right) \\
\text { Location on a map }\end{array}$ & Small afforested catchments & Yes & 1983-2006 & $\begin{array}{l}\text { Weekly until } 2003 \text {, } \\
\text { thereafter fortnightly }\end{array}$ & Loch Ard data in [42] \\
\hline
\end{tabular}


Table 1. Cont

\begin{tabular}{|c|c|c|c|c|c|c|c|c|c|}
\hline \multirow[t]{2}{*}{ Ref. } & \multicolumn{6}{|c|}{ System } & \multirow[t]{2}{*}{ Period } & \multirow{2}{*}{$\begin{array}{l}\text { Sampling } \\
\text { frequency }\end{array}$} & \multirow[t]{2}{*}{ Data source } \\
\hline & Type $^{\text {a }}$ & No. & Country & Details & General characteristics & $\begin{array}{l}\text { Acid rain } \\
\text { recovery }^{b}\end{array}$ & & & \\
\hline$[52]$ & streams & 3 & Norway & $\begin{array}{l}\text { Birkenes (B), Storgama (S), } \\
\text { Langtjern (L) } \\
\text { No map }\end{array}$ & $\begin{array}{l}\text { Severely acidified systems; } \\
\text { forested, undisturbed } \\
\text { Catchment area: } 0.41-0.8 \mathrm{~km}^{2}\end{array}$ & Yes & 1985-2003 & $\begin{array}{l}\text { B: daily } \\
\text { S, L: >1992 weekly; } \\
<1992 \text { daily }\end{array}$ & $\begin{array}{l}\text { Norwegian program for } \\
\text { monitoring long-range } \\
\text { transported air pollutants }\end{array}$ \\
\hline [53] & $\begin{array}{l}\text { lakes } \\
\text { and } \\
\text { rivers }\end{array}$ & 117 & $\begin{array}{l}\text { United } \\
\text { Kingdom }\end{array}$ & No list, no map & & Yes & 1977-2002 & $\begin{array}{l}\text { HMS data: some } \\
\text { weekly, most monthly }\end{array}$ & $\begin{array}{l}\text { Harmonised Monitoring } \\
\text { Scheme database (HMS) } \\
\mathbf{1 9 8} \text { sites from [54] also } \\
\text { considered }\end{array}$ \\
\hline$[55]$ & $\begin{array}{l}\text { lakes } \\
\text { streams }\end{array}$ & $\begin{array}{l}12 \\
5\end{array}$ & USA & $\begin{array}{l}\text { Adirondack lakes (AL) and } \\
\text { Catskill streams (CS), New } \\
\text { York } \\
\text { List of systems in a table, } \\
\text { location on a map }\end{array}$ & $\begin{array}{l}\text { CS streams chosen in the most } \\
\text { sensitive to acidification } \\
\text { areas; } \\
\text { AL lakes: only drainage lakes } \\
\text { with retention time }<6 \\
\text { months }\end{array}$ & Yes & 1992-2001 & $\begin{array}{l}\text { Lakes: monthly } \\
\text { Streams: variable }\end{array}$ & $\begin{array}{l}\text { Adirondack lakes: selected } \\
\text { from the } 52 \text { in the } \\
\text { Adirondack Long-Term } \\
\text { Monitoring (ALTM) }\end{array}$ \\
\hline$[15]$ & $\begin{array}{l}\text { lakes } \\
\text { streams }\end{array}$ & $\begin{array}{l}11 \\
11\end{array}$ & $\begin{array}{l}\text { United } \\
\text { Kingdom }\end{array}$ & $\begin{array}{l}\text { List of sites in a table, no } \\
\text { map }\end{array}$ & $\begin{array}{l}\text { Located in the main acid- } \\
\text { sensitive regions of the UK, } \\
\text { mostly moorland }\end{array}$ & Yes & 1988-2003 & $\begin{array}{l}\text { Lakes: quarterly } \\
\text { Streams: monthly }\end{array}$ & Same data as in [16] \\
\hline [48] & $\begin{array}{l}\text { lakes } \\
\text { streams }\end{array}$ & $\begin{array}{l}13 \\
2\end{array}$ & Finland & $\begin{array}{l}\text { List of systems in a table, } \\
\text { location on a map }\end{array}$ & $\begin{array}{l}\text { Small forest lakes and forest } \\
\text { streams } \\
\text { Lake area: } 0.024-1.62 \mathrm{~km}^{2} \\
\text { Depth: } 4.7-19.5 \mathrm{~m} \\
\text { Catchment area: } 0.28-4.36 \\
\mathrm{~km}\end{array}$ & Yes & $1987-2003$ & $\begin{array}{l}\text { ICP lakes: } 1 \text { sample } \\
\text { winter and summer, } \\
2 \text { spring, and fall } \\
\text { IM: } 8-12 \text { samples per } \\
\text { year }\end{array}$ & $\begin{array}{l}10 \text { lakes Regional } \\
\text { Monitoring Network of } \\
\text { Lake acidification } \\
\text { (RMLA), } 3 \text { lakes ICP } \\
\text { Integrated Monitoring } \\
\text { program (ICP UM) }\end{array}$ \\
\hline
\end{tabular}


Table 1. Cont

\begin{tabular}{|c|c|c|c|c|c|c|c|c|c|}
\hline \multirow[t]{2}{*}{ Ref. } & \multicolumn{6}{|c|}{ System } & \multirow[t]{2}{*}{ Period } & \multirow{2}{*}{$\begin{array}{l}\text { Sampling } \\
\text { frequency }\end{array}$} & \multirow[t]{2}{*}{ Data source } \\
\hline & Type $^{\text {a }}$ & No. & Country & Details & General characteristics & $\begin{array}{l}\text { Acid rain } \\
\text { recovery? }^{b}\end{array}$ & & & \\
\hline [31] & stream & $\begin{array}{l}1 \\
(2 \text { sampling } \\
\text { sites })\end{array}$ & $\begin{array}{l}\text { United } \\
\text { Kingdom }\end{array}$ & $\begin{array}{l}\text { River Tees (Moor House): } \\
\text { Trout Beck and Cottage Hill } \\
\text { Sike } \\
\text { Location on a map }\end{array}$ & Blanket peat catchment & NM & 1994-2001 & Weekly & $\begin{array}{l}\text { Trout Beck data already } \\
\text { published in [30] }\end{array}$ \\
\hline$[56]$ & lakes & 7 & $\begin{array}{l}\text { Ontario, } \\
\text { Canada }\end{array}$ & $\begin{array}{l}\text { Dorset region: } \\
7 \text { lakes (Blue Chalk, Chub, } \\
\text { Crosson, Dickie, Harp, } \\
\text { Plastic, Red Chalk) and their } \\
20 \text { subcatchments } \\
\text { Location on a map }\end{array}$ & $\begin{array}{l}\text { Forested, oligotrophic and } \\
\text { mesotrophic lakes } \\
\text { Lake area: } 0.3214-0.9360 \mathrm{~km}^{2} \\
\text { Mean depth: } 7.9-14.2 \mathrm{~m} \\
\text { Catchment area: } \\
0.955-5.324 \mathrm{~km}^{2}\end{array}$ & Yes & 1978-1998 & 1 to 4 week intervals & - \\
\hline [16] & $\begin{array}{l}\text { lakes } \\
\text { streams }\end{array}$ & $\begin{array}{l}11 \\
11\end{array}$ & $\begin{array}{l}\text { United } \\
\text { Kingdom }\end{array}$ & List of sites, no map & $\begin{array}{l}\text { Located in the main acid- } \\
\text { sensitive regions of the UK, } \\
\text { mostly moorland }\end{array}$ & Yes & $1988-2003$ & $\begin{array}{l}\text { Lakes: quarterly } \\
\text { Streams: monthly }\end{array}$ & $\begin{array}{l}\text { UK Acid Waters } \\
\text { Monitoring } \\
\text { Network (AWMN) }\end{array}$ \\
\hline [57] & river & $\begin{array}{l}1 \\
\text { (6 stations) }\end{array}$ & USA & $\begin{array}{l}\text { Hudson River (New York), } \\
\text { sampling points: } \mathrm{km} 146 \text { and } \\
5 \text { stations km } 63 \text { to } 222 \\
\text { Location on a map }\end{array}$ & Total catchment: $21,034 \mathrm{~km}^{2}$ & NM & $1988-2003$ & $\begin{array}{l}\text { Fortnightly (km 146), } \\
\text { every } 2 \text { months } \\
\text { (longitudinal series) } \\
\text { in other points }\end{array}$ & - \\
\hline [23] & streams & $\begin{array}{l}3 \\
(6 \text { sampling } \\
\text { sites })\end{array}$ & $\begin{array}{l}\text { Wales, } \\
\text { United } \\
\text { Kingdom }\end{array}$ & $\begin{array}{l}\text { Upper River Severn } \\
\text { catchments (Plynlimon): } \\
\text { Upper Hafren, Upper Hore, } \\
\text { Lower Hafren, Lower Hore, } \\
\text { Nant Tanllwyth, South2Hore } \\
\text { No map }\end{array}$ & $\begin{array}{l}\text { Catchments: } 3580 \text { km² (Hafren), }^{2} \\
3172 \text { (Hore), } 0.916 \text { (Tanllwyth) }\end{array}$ & Yes & $\begin{array}{l}(1983,1984, \\
1988,1990 \\
1991)-2002\end{array}$ & Weakly or fortnightly & $\begin{array}{l}\text { Centre for Ecology and } \\
\text { Hydrology (CEH) }\end{array}$ \\
\hline
\end{tabular}


Table 1. Cont

\begin{tabular}{|c|c|c|c|c|c|c|c|c|c|}
\hline \multirow[t]{2}{*}{ Ref. } & \multicolumn{6}{|c|}{ System } & \multirow[t]{2}{*}{ Period } & \multirow{2}{*}{$\begin{array}{l}\text { Sampling } \\
\text { frequency }\end{array}$} & \multirow[t]{2}{*}{ Data source } \\
\hline & Type $^{\text {a }}$ & No. & Country & Details & General characteristics & $\begin{array}{l}\text { Acid rain } \\
\text { recovery? }{ }^{b}\end{array}$ & & & \\
\hline$[18]$ & sites & 189 & $\begin{array}{l}\text { Europe } \\
\text { and North } \\
\text { America }\end{array}$ & $\begin{array}{l}\text { Europe: Alps (6), } \\
\text { East Central Europe (20), } \\
\text { Northern Nordic (7), } \\
\text { Southern Nordic (19), } \\
\text { UK/Ireland (9), } \\
\text { West Central Europe (12) } \\
\text { N. America: Maine/Atlantic } \\
\text { Canada (18), } \\
\text { Vermont/Quebec (15), } \\
\text { Adirondacks (48), } \\
\text { Appalachian Plateau (9), } \\
\text { Upper Midwest (23), } \\
\text { Virginia Blue Ridge (3) } \\
\text { List of sites in [9], } \\
\text { approximate location on a map }\end{array}$ & $\begin{array}{l}\text { Regions defined based on } \\
\text { similar acid-sensitivity and } \\
\text { rates of deposition }\end{array}$ & Yes & 1990-2001 & Variable & $\begin{array}{l}\text { International Cooperative } \\
\text { Programme on Assessment } \\
\text { and Monitoring of } \\
\text { Acidification of Rivers and } \\
\text { Lakes (ICP) } \\
\text { Probably some data } \\
\text { already considered in } \\
\text { other studies }\end{array}$ \\
\hline [58] & rivers & 16 & Finland & $\begin{array}{l}\text { List of rivers in a table, } \\
\text { location on a map }\end{array}$ & $\begin{array}{l}\text { Vegetation: from boreal taiga } \\
\text { to sub-arctic vegetation } \\
\text { Mean annual discharge } \\
\left(\mathrm{m}^{3} \mathrm{~s}^{-1}\right): 3>100,520-100 \\
8<20\end{array}$ & NM & $\begin{array}{l}1975-2000, \\
\text { shorter for } 5 \\
\text { rivers }\end{array}$ & Monthly & $\begin{array}{l}\text { Finnish Environmental } \\
\text { Institute (FEI) or regional } \\
\text { environment centres }\end{array}$ \\
\hline [59] & lake & 1 & Norway & $\begin{array}{l}\text { Lake Elvåga in Østmarka area } \\
\text { Location on a map }\end{array}$ & $\begin{array}{l}\text { Forest area bordering Oslo } \\
\text { city district } \\
\text { Lake area: } 1 \mathrm{~km}^{2} \\
\text { Samples from } 40 \mathrm{~m} \text { depth }\end{array}$ & Yes & $\begin{array}{l}\text { From: } \\
-1976 \text { (color) } \\
-1982(\mathrm{COD}) \\
-1988 \text { (DOC) } \\
\text { to } 2002\end{array}$ & No information & $\begin{array}{l}\text { Oslo Water and Sewage } \\
\text { Works }\end{array}$ \\
\hline
\end{tabular}


Table 1. Cont

\begin{tabular}{|c|c|c|c|c|c|c|c|c|c|}
\hline \multirow[t]{2}{*}{ Ref. } & \multicolumn{6}{|c|}{ System } & \multirow[t]{2}{*}{ Period } & \multirow{2}{*}{$\begin{array}{l}\text { Sampling } \\
\text { frequency }\end{array}$} & \multirow[t]{2}{*}{ Data source } \\
\hline & Type $^{\text {a }}$ & No. & Country & Details & General characteristics & $\begin{array}{l}\text { Acid rain } \\
\text { recovery }^{b}\end{array}$ & & & \\
\hline [47] & $\begin{array}{l}\text { lakes } \\
\text { supply } \\
\text { reservoirs } \\
\text { streams } \\
\text { and rivers }\end{array}$ & $\begin{array}{l}29 \\
8\end{array}$ & $\begin{array}{l}\text { United } \\
\text { Kingdom }\end{array}$ & $\begin{array}{l}\text { List of sites in a table, } \\
\text { location on a map }\end{array}$ & Catchments: $400 \mathrm{~m}^{2}-2120 \mathrm{~km}^{2}$ & $\begin{array}{l}\text { Many sites, } \\
\text { yes }\end{array}$ & $\begin{array}{l}\text { variable-2000; } \\
\text { some from } \\
\text { 1962, most } 10 \\
\text { years long }\end{array}$ & Variable & $\begin{array}{l}\text { Sites from: Freshwater } \\
\text { Laboratory; Scottish EPA; } \\
\text { North Pennines; } \\
\text { UKAWMN; CEH; } \\
\text { Yorkshire Water reservoirs; } \\
\text { ECN- Forestry } \\
\text { Commission } \\
\text { Data in [37] included }\end{array}$ \\
\hline [30] & stream & 1 & $\begin{array}{l}\text { United } \\
\text { Kingdom }\end{array}$ & $\begin{array}{l}\text { River Tees (Trout Beck) } \\
\text { Location on a map }\end{array}$ & $\begin{array}{l}\text { Blanket peat catchment } \\
\text { Catchment: } 11.4 \mathrm{~km}^{2}\end{array}$ & NM & $1992-2000$ & Weekly & $\begin{array}{l}\text { UK Environmental Change } \\
\text { Network (ECN) }\end{array}$ \\
\hline [60] & rivers & 2 & $\begin{array}{l}\text { United } \\
\text { Kingdom }\end{array}$ & $\begin{array}{l}\text { Rivers Tees (Broken Scar), } \\
\text { Coquet (Warkworth) } \\
\text { Location on a map }\end{array}$ & $\begin{array}{l}\text { Rivers draining upland peat, } \\
\text { low flood waves ( } 2 \text { days) }\end{array}$ & NM & $\begin{array}{l}\text { Tees: } 1970- \\
2000 \\
\text { Coquet: } 1962- \\
2001\end{array}$ & See $[61]$ & Same data as in [61] \\
\hline [62] & lakes & $\begin{array}{l}52 \\
(48 \text { not } \\
\text { limed) }\end{array}$ & USA & $\begin{array}{l}\text { Adirondack Lakes, } \\
\text { New York } \\
\text { List of lakes in a table, } \\
\text { no map }\end{array}$ & $\begin{array}{l}\text { Watersheds largely forested, } \\
\text { with hardwood or mixed } \\
\text { vegetation } \\
\text { Lake area: } 0.008-5.125 \mathrm{~km}^{2} \\
\text { Max depth: } 1.2-32.0 \mathrm{~m}\end{array}$ & Yes & $\begin{array}{l}\text { 1982-2000 (17 } \\
\text { lakes), 1992- } \\
2000 \text { (52 lakes) }\end{array}$ & Monthly & $\begin{array}{l}\text { Adirondack Long-Term } \\
\text { Monitoring (ALTM) } \\
\text { program lakes } \\
\text { Some data in [63] }\end{array}$ \\
\hline [64] & lakes & 163 & Finland & No list, location on a map & $\begin{array}{l}\text { Forested catchments. All acid } \\
\text { sensitive. } \\
\text { Small (median area: } 0.1 \mathrm{~km}^{2} \text { ), } \\
\text { headwater or seepage lakes }\end{array}$ & Yes & $1990-1999$ & Each autumn & $\begin{array}{l}\text { Finnish acidification } \\
\text { monitoring lake network } \\
\text { (RMLA) }\end{array}$ \\
\hline
\end{tabular}


Table 1. Cont

\begin{tabular}{|c|c|c|c|c|c|c|c|c|c|}
\hline \multirow[t]{2}{*}{ Ref. } & \multicolumn{6}{|c|}{ System } & \multirow[t]{2}{*}{ Period } & \multirow{2}{*}{$\begin{array}{l}\text { Sampling } \\
\text { frequency }\end{array}$} & \multirow[t]{2}{*}{ Data source } \\
\hline & Type $^{\mathrm{a}}$ & No. & Country & Details & General characteristics & $\begin{array}{l}\text { Acid rain } \\
\text { recovery? }^{\text {b }}\end{array}$ & & & \\
\hline [65] & stream & 1 & $\begin{array}{l}\text { Czech } \\
\text { Republic }\end{array}$ & Malše River, no map & $\begin{array}{l}\text { Upland stream } \\
\text { Catchment area: } 438 \mathrm{~km}^{2}\end{array}$ & No & $1969-2000$ & Daily & $\begin{array}{l}\text { Waterworks Pořešín (Water } \\
\text { Supply and Sewage South } \\
\text { Bohemia) }\end{array}$ \\
\hline [35] & lakes & 9 & $\begin{array}{l}\text { Ontario, } \\
\text { Canada }\end{array}$ & $\begin{array}{l}\text { Lakes: Blue Chalk, Chub, } \\
\text { Crosson, Dickie, Harp, } \\
\text { Heney, Plastic, Red Chalk } \\
\text { (two basins), located } 150 \\
\text { km N of Toronto } \\
\text { Location on a map }\end{array}$ & $\begin{array}{l}\text { Oligotrophic } \\
\text { Lake area: } 0.13-0.94 \mathrm{~km}^{2} \\
\text { Max depth: } 6-38 \mathrm{~m} \\
\text { Renewal time: } 1.1-7.7 \mathrm{y} \\
\text { Catchment area: } 0.93-5.89 \mathrm{~km}^{2}\end{array}$ & Yes & $1978-1998$ & $\begin{array}{l}\text { 5-24 times per } \\
\text { year during the } \\
\text { ice-free period }\end{array}$ & - \\
\hline [66] & lakes & 705 & Canada & $\begin{array}{l}\text { No list of sites, } \\
\text { incomplete map } \\
\text { Quebec, } 33 \text { lakes } \\
\text { Ontario, } 662 \text { lakes }\end{array}$ & & Yes & $\begin{array}{l}\text { Quebec: } \\
\text { 1990-1997 } \\
\text { Ontario: } \\
\text { 1990-1999 }\end{array}$ & No information & $\begin{array}{l}\text { Many different sources, } \\
\text { detailed in the article; most } \\
\text { Ontario lakes from the } \\
\text { Canadian Wildlife Service } \\
\text { (CWS) }\end{array}$ \\
\hline [67] & lakes & 8 & $\begin{array}{l}\text { Ontario, } \\
\text { Canada }\end{array}$ & $\begin{array}{l}\text { Lakes: Bell, David, George, } \\
\text { Johnnie, Killarney, Nellie, } \\
\text { OSA, Ruth-Roy in } \\
\text { Killarney Park, near } \\
\text { Sudbury } \\
\text { Location on a map }\end{array}$ & $\begin{array}{l}\text { Strongly affected by } \\
\text { acidification }\end{array}$ & Yes & $1988-2001$ & $\begin{array}{l}\text { Annually in } \\
\text { midsummer }\end{array}$ & $\begin{array}{l}\text { Several sources given, not } \\
\text { clear which one } \\
\text { corresponds to OC data }\end{array}$ \\
\hline [41] & $\begin{array}{l}\text { lake } \\
\text { streams }\end{array}$ & $\begin{array}{l}1 \\
8 \text { (one } \\
\text { with } \\
7 \text { sites) }\end{array}$ & $\begin{array}{l}\text { Scotland, } \\
\text { United } \\
\text { Kingdom }\end{array}$ & $\begin{array}{l}\text { Loch Ard: } 2 \text { streams: } \\
\text { Corrie burn (1 site), Burns } \\
\text { ( } 7 \text { sites) } \\
\text { Loch Grannoch: } 6 \text { streams } \\
\text { and } 1 \text { loch }\end{array}$ & $\begin{array}{l}\text { Loch Grannoch catchment area: } \\
15.45 \mathrm{~km}^{2}\end{array}$ & Yes & $\begin{array}{l}\text { Loch Ard: } \\
\text { 1977-2000 } \\
\text { Loch Grannoch: } \\
\text { 1978-present }\end{array}$ & $\begin{array}{l}\text { Loch Ard: weekly } \\
\text { or fortnightly } \\
\text { Loch Grannoch: } \\
\text { variable }\end{array}$ & - \\
\hline
\end{tabular}


Table 1. Cont

\begin{tabular}{|c|c|c|c|c|c|c|c|c|c|}
\hline \multirow[t]{2}{*}{ Ref. } & \multicolumn{6}{|c|}{ System } & \multirow[t]{2}{*}{ Period } & \multirow{2}{*}{$\begin{array}{l}\text { Sampling } \\
\text { frequency }\end{array}$} & \multirow[t]{2}{*}{ Data source } \\
\hline & Type $^{a}$ & No. & Country & Details & General characteristics & $\begin{array}{l}\text { Acid rain } \\
\text { recovery? }^{b}\end{array}$ & & & \\
\hline [61] & rivers & 3 & $\begin{array}{l}\text { United } \\
\text { Kingdom }\end{array}$ & $\begin{array}{l}\text { Rivers Tees (Broken Scar), } \\
\text { Wear (Wearhead), } \\
\text { Coquet (Warkworth) } \\
\text { Location on a map }\end{array}$ & Rivers draining upland peat & $\mathrm{NM}$ & $\begin{array}{l}\text { Tees: 1970-2000 } \\
\text { Wear: 1969-1998 } \\
\text { Coquet: } \\
\text { 1962-2000 }\end{array}$ & $\begin{array}{l}\text { Tees: daily } \\
\text { Wear: variable } \\
\text { Coquet: initially } \\
\text { daily, then weekly }\end{array}$ & - \\
\hline [14] & $\begin{array}{l}\text { lakes } \\
\text { streams }\end{array}$ & $\begin{array}{l}11 \\
11\end{array}$ & $\begin{array}{l}\text { United } \\
\text { Kingdom }\end{array}$ & No list, no map & $\begin{array}{l}\text { Freshwater draining upland } \\
\text { catchments (peatlands) }\end{array}$ & Yes & 1989-2000 & $\begin{array}{l}\text { Lakes: quarterly } \\
\text { Streams: monthly }\end{array}$ & $\begin{array}{l}\text { UK Acid Waters } \\
\text { Monitoring Network } \\
(\mathrm{AWMN})\end{array}$ \\
\hline [68] & lakes & 4 & $\begin{array}{l}\text { Ontario, } \\
\text { Canada }\end{array}$ & $\begin{array}{l}\text { Lakes Nellie, OSA, George and } \\
\text { Bell in Killarney Park } \\
\text { Location on a map }\end{array}$ & $\begin{array}{l}\text { All acidification recovery sites } \\
\text { Lake surface area: } \\
1.885-3.474 \mathrm{~km}^{2} \\
\text { Max depth: } 26.8-54.9 \mathrm{~m} \\
\end{array}$ & Yes & 1969-1999 & Not given & $\begin{array}{l}\text { Killarney Park is a } \\
\text { Canadian EMAN } \\
\text { (Ecological Monitoring and } \\
\text { Assessment Network) site }\end{array}$ \\
\hline [69] & $\begin{array}{l}\text { lakes } \\
\text { streams }\end{array}$ & $\begin{array}{l}21 \\
16\end{array}$ & $\begin{array}{l}\text { Scotland, } \\
\text { United } \\
\text { Kingdom }\end{array}$ & $\begin{array}{l}\text { Classified in four geographical } \\
\text { areas } \\
\text { List in a table, location on a map }\end{array}$ & Moorland and forested sites & Yes & $\begin{array}{l}(1972-1988)- \\
2000\end{array}$ & $\begin{array}{l}\text { Variable, described } \\
\text { in detail in the } \\
\text { article }\end{array}$ & Freshwater Laboratory \\
\hline [70] & $\begin{array}{l}\text { "ICP } \\
\text { Waters" } \\
\text { sites }\end{array}$ & 98 & $\begin{array}{l}\text { Europe and } \\
\text { North } \\
\text { America }\end{array}$ & No list, no map & $\begin{array}{l}\text { Only acidification sensitive } \\
\text { sites included }\end{array}$ & Yes & 1989-1998 & $\begin{array}{l}\text { At least } 2 \text { periods } \\
\text { per year }\end{array}$ & $\begin{array}{l}\text { International Cooperative } \\
\text { Programme (ICP) on } \\
\text { Assessment and Monitoring } \\
\text { of Acidification of Rivers } \\
\text { and Lakes }\end{array}$ \\
\hline [71] & lakes & 344 & Scandinavia & $\begin{array}{l}163 \text { Finland, } 100 \text { Norway, } 81 \\
\text { Sweden } \\
\text { No list, map }\end{array}$ & $\begin{array}{l}\text { Finland and Norway: headwater } \\
\text { or seepage lakes, no pollution } \\
\text { Sweden: forested areas, no } \\
\text { pollution }\end{array}$ & Yes & 1990-1999 & $\begin{array}{l}\text { Once annually } \\
\text { (autumn) }\end{array}$ & $\begin{array}{l}\text { National Monitoring } \\
\text { Programs of Norway, } \\
\text { Finland and Sweden (subset } \\
\text { from the } 5690 \text { lakes in the } \\
\text { Northern European lake } \\
\text { survey of 1995) }\end{array}$ \\
\hline
\end{tabular}


Table 1. Cont

\begin{tabular}{|c|c|c|c|c|c|c|c|c|c|}
\hline \multirow[t]{2}{*}{ Ref. } & \multicolumn{6}{|c|}{ System } & \multirow[t]{2}{*}{ Period } & \multirow{2}{*}{$\begin{array}{l}\text { Sampling } \\
\text { frequency }\end{array}$} & \multirow[t]{2}{*}{ Data source } \\
\hline & Type $^{\text {a }}$ & No. & Country & Details & General characteristics & $\begin{array}{l}\text { Acid rain } \\
\text { recovery? }^{\text {b }}\end{array}$ & & & \\
\hline [72] & rivers & 9 & Latvia & $\begin{array}{l}\text { Rivers Venta, Tebra, } \\
\text { Lielupe, Iecava, Misa, } \\
\text { Daugava, Dubna, Gauja, } \\
\text { Tuliya } \\
\text { Location on a map }\end{array}$ & Drainage area: $33-70,600 \mathrm{~km}^{2}$ & NM & $1977-1995$ & Monthly & $\begin{array}{l}\text { Latvian } \\
\text { Hydrometeorological } \\
\text { Agency }\end{array}$ \\
\hline [73] & lakes & 161 & $\begin{array}{l}\text { Ontario, } \\
\text { Canada }\end{array}$ & $\begin{array}{l}\text { Sudbury region } \\
\text { No list, no map }\end{array}$ & $\begin{array}{l}\text { Acid stressed lakes } \\
\text { Lake surface area: } 0.001-3.50 \mathrm{~km}^{2} \\
\text { Depth: } 0.6-22.2 \mathrm{~m}\end{array}$ & Yes & 1983-1995 & Annually & - \\
\hline [74] & lakes & $\begin{array}{l}51 \text { but } \\
\text { only } 37 \\
\text { used for } \\
\text { OC trend } \\
\text { analysis }\end{array}$ & $\begin{array}{l}\text { Quebec, } \\
\text { Canada }\end{array}$ & $\begin{array}{l}\mathrm{N} \text { of St. Lawrence River } \\
\text { between Ottawa River and } \\
\text { Baie Comeau; divided in } 7 \\
\text { chemically homogeneous } \\
\text { regions } \\
\text { No list, location on a map } \\
\end{array}$ & $\begin{array}{l}\text { Headwater lakes } \\
\text { Lake area: } 0.13-0.57 \mathrm{~km}^{2} \\
\text { Mean depth: } 10.2-28.0 \mathrm{~m} \\
\text { Renewal time: } 12.7-63.6 \text { months } \\
\text { Values quoted are region means }\end{array}$ & $\begin{array}{l}\text { Yes } \\
\text { (those not } \\
\text { affected, } \\
\text { excluded) }\end{array}$ & $\begin{array}{l}(1983,1986, \\
1989)-1993\end{array}$ & $\begin{array}{l}\text { 17: } 6 \text { times a year } \\
\text { 20: twice a year } \\
\text { 14: once a year }\end{array}$ & Environment Canada \\
\hline [75] & $\begin{array}{l}\text { lakes } \\
\text { streams }\end{array}$ & $\begin{array}{l}3 \\
4\end{array}$ & $\begin{array}{l}\text { Ontario, } \\
\text { Canada }\end{array}$ & $\begin{array}{l}\text { Experimental Lakes Area, } \\
\text { lakes number 239, 240, 302S } \\
\text { and inflowing streams and } \\
\text { lake outflows; } \\
\text { NW Ontario } \\
\text { Location on a map }\end{array}$ & $\begin{array}{l}\text { Lake area: } 0.543,0.442,0.109 \mathrm{~km}^{2} \\
\text { Mean depth: } 10.9,6.0,5.1 \mathrm{~m} \\
\text { Renewal time: } 4-26,<1-6,4-12 \mathrm{y} \\
\text { Lake } 302 \mathrm{~S} \text { artificially acidified to } \\
\text { pH } 4.5\end{array}$ & Yes & $\begin{array}{l}\text { 239: 1972-1990 } \\
\text { 240: summers } \\
\text { 1972, 1975-1978, } \\
\text { 1984-1990; all } \\
\text { winters, except } \\
\text { 1972-1974 and } \\
\text { 1976-1981 } \\
\text { 302: 1981-1990 } \\
\text { Inflow streams: } \\
\text { 1970-1990 }\end{array}$ & $\begin{array}{l}\text { Lakes: monthly or } \\
\text { more frequently } \\
\text { during the ice-free } \\
\text { season and 2-4 times } \\
\text { in winter } \\
\text { Streams: weekly }\end{array}$ & $\begin{array}{l}\text { Experimental Lakes Area } \\
\text { (ELA) }\end{array}$ \\
\hline
\end{tabular}


Table 1. Cont.

\begin{tabular}{|c|c|c|c|c|c|c|c|c|c|}
\hline \multirow[t]{2}{*}{ Ref. } & \multicolumn{6}{|c|}{ System } & \multirow[t]{2}{*}{ Period } & \multirow{2}{*}{$\begin{array}{l}\text { Sampling } \\
\text { frequency }\end{array}$} & \multirow[t]{2}{*}{ Data source } \\
\hline & Type $^{\text {a }}$ & No. & Country & Details & General characteristics & $\begin{array}{l}\text { Acid rain } \\
\text { recovery? }^{\text {b }}\end{array}$ & & & \\
\hline [63] & lakes & 17 & USA & $\begin{array}{l}\text { Adirondack Lakes, New York } \\
\text { List of lakes in a table, no map }\end{array}$ & $\begin{array}{l}15 \text { drainage lakes, } 2 \text { seepage lakes } \\
\text { Lake surface area: } 0.01-5.035 \mathrm{~km}^{2} \\
\text { Max depth: } 4-24 \mathrm{~m} \\
\text { Retention time: } 0.03-2.5 \mathrm{y}\end{array}$ & Yes & $1982-1991$ & Monthly & $\begin{array}{l}\text { Adirondack Long-Term } \\
\text { Monitoring (ALTM) } \\
\text { program lakes }\end{array}$ \\
\hline [76] & $\begin{array}{l}\text { lake } \\
\text { rivers }\end{array}$ & $\begin{array}{l}1 \\
7\end{array}$ & Sweden & $\begin{array}{l}\text { Lake Öjaren } \\
\text { Rivers: Ore Älv, Ljusnan, West } \\
\text { Dalälven, Hedströmmen, } \\
\text { Alsterälven, Nissan, Lyckebyän } \\
\text { Location on a map }\end{array}$ & $\begin{array}{l}\text { Lake surface area: } 20 \mathrm{~km}^{2}, \\
\text { max depth } 9 \mathrm{~m} \\
\text { River catchments: } 365-8493 \mathrm{~km}^{2}\end{array}$ & Yes & $\begin{array}{l}\text { Lake: } 1960-1988 \\
\text { River Alsterälven: } \\
\text { 1966-1987 }\end{array}$ & $\begin{array}{l}\text { Lake: } 6 \text { times per } \\
\text { year } \\
\text { Rivers: monthly }\end{array}$ & $\begin{array}{l}\text { Surface-water monitoring } \\
\text { program of the Swedish } \\
\text { Environmental Protection } \\
\text { Board }\end{array}$ \\
\hline [77] & $\begin{array}{l}\text { lakes } \\
\text { rivers }\end{array}$ & $\begin{array}{l}283 \\
18\end{array}$ & Sweden & $\begin{array}{l}\text { List of rivers in a table } \\
\text { Location on a map }\end{array}$ & $\begin{array}{l}\text { Lake median size: } 2 \mathrm{~km}^{2} \\
\text { River drainage area: } \\
25-10,797 \mathrm{~km}^{2} \text {; mean discharge: } \\
4.0-146.3 \mathrm{~m}^{3} \mathrm{~s}^{-1}\end{array}$ & NM & $\begin{array}{l}\text { Lakes: } 1972-1987 \\
\text { Rivers: } 1972-1986 \\
\text { (some 1965) }\end{array}$ & $\begin{array}{l}\text { Lakes: lower than } \\
\text { in rivers } \\
\text { Rivers: monthly }\end{array}$ & $\begin{array}{l}\text { Databases: } \\
\text { - Lakes -Long Term } \\
\text { Variation (LLTV) } \\
\text { - Running Waters Data } \\
\text { Base (RWDB) }\end{array}$ \\
\hline [78] & lakes & 4 & Sweden & $\begin{array}{l}\text { Lakes Oxsjön (OX), } \\
\text { Hammardammen (HA), Innaren } \\
\text { (IN), Värmen (VAAll in South } \\
\text { Sweden } \\
\text { No map }\end{array}$ & $\begin{array}{l}\text { Forest lakes } \\
\text { Lake surface area: } 0.90-16.4 \mathrm{~km}^{2} \\
\text { Max depth: } 2-19 \mathrm{~m} \\
\text { Catchment area: } \\
10.2-200 \mathrm{~km}^{2}\end{array}$ & Yes & $\begin{array}{l}\text { OX: 1967-1982 } \\
\text { HA: 1972-1988 } \\
\text { IN: 1970's-1980's } \\
\text { VA: 1976-1986 }\end{array}$ & $\begin{array}{l}\text { OX: 3-4 per year } \\
\text { (vegetative season) } \\
\text { HA: daily } \\
\text { IN: } 37 \text { times in } \\
\text { 1970's; } 25,1980 \text { 's } \\
\text { VA: } 4 \text { per year }\end{array}$ & - \\
\hline [79] & stream & 1 & $\begin{array}{l}\text { United } \\
\text { Kingdom }\end{array}$ & $\begin{array}{l}\text { Raw waters arriving at Chellow } \\
\text { Heights treatment works, Upper } \\
\text { Nidderdale, North Yorkshire } \\
\text { Location on a map }\end{array}$ & $\begin{array}{l}\text { Much of the water from Angram } \\
\text { and Scar House reservoirs }\end{array}$ & & 1979-1987 & $\begin{array}{l}\text { From daily to less } \\
\text { than weekly }\end{array}$ & Yorkshire Water \\
\hline
\end{tabular}

Notes: ${ }^{\mathrm{a}}$ The distinction between streams and rivers is not always well-defined, the denomination used by the authors has been kept; ${ }^{\mathrm{b}} \mathrm{NM}=$ not mentioned. 
Table 2. Published studies containing long-term organic carbon concentration temporal trends in freshwaters. Methodological information.

\begin{tabular}{|c|c|c|c|c|c|c|c|c|}
\hline Ref. & Filtration & $\begin{array}{l}\text { OC quantification } \\
\text { method }\end{array}$ & $\begin{array}{l}\text { Original data } \\
\text { plotted? }\end{array}$ & $\begin{array}{l}\text { OC range }{ }^{a} \\
/ \mathrm{mg} \mathrm{C} \mathrm{L}^{-1}\end{array}$ & $\begin{array}{l}\text { Types of } \\
\text { OC }\end{array}$ & Lake sampling & Data transformation & Statistical treatment \\
\hline [20] & $\begin{array}{l}\text { Filtered ( } 0.45 \\
\mu \text { m cellulose } \\
\text { nitrate filters) }\end{array}$ & $\begin{array}{l}\text { TOC, DOC: } \\
\text { >1997: HTC (DIN 38409-H3-1) } \\
\text { <1997: no documentation } \\
\text { POC: probably by difference }\end{array}$ & $\begin{array}{l}\text { Mean annual } \\
{[\mathrm{TOC}],[\mathrm{POC}]} \\
\text { [DOC] vs. time } \\
\text { shown }\end{array}$ & & & & $\begin{array}{l}\text { Mean annual values } \\
\text { shown, not clear } \\
\text { how many sampling } \\
\text { points have been } \\
\text { averaged }\end{array}$ & $\begin{array}{l}\text { No mathematical } \\
\text { treatment }\end{array}$ \\
\hline [21] & Not mentioned & $\begin{array}{l}\text { DOC, UV-persulfate oxidation } \\
\text { followed by IR }\end{array}$ & $\begin{array}{l}\text { Mean }[\mathrm{DOC}] v s . \\
\text { time shown for } \\
\text { all lakes }\end{array}$ & $\begin{array}{c}\text { Mean range: } \\
2.05-8.38\end{array}$ & & $\begin{array}{l}\text { Integrated } 0-5 \mathrm{~m} \text { samples } \\
\text { collected from the middle of } \\
\text { the lake; when }<1 \mathrm{~m} \text { deep, } \\
\text { drawn } 1 \mathrm{~m} \text { from the bottom to } \\
\text { the surface }\end{array}$ & No, original data used & $\begin{array}{l}\text { Trends: SMK } \\
\text { Slope: magnitude of } \\
\text { linear trend with } \\
\text { DETECT software } \\
\text { (reference given) }\end{array}$ \\
\hline$[22]$ & No information & DOC, no information & No & Median: 2.1 & & & $\begin{array}{l}\text { Probably all data } \\
\text { used }\end{array}$ & $\begin{array}{l}\text { Trends: Integrated } \\
\text { Random Walk } \\
\text { analysis }\end{array}$ \\
\hline [24] & No information & DOC, no information & $\begin{array}{l}\text { Mean monthly } \\
\text { [DOC] vs. time } \\
\text { shown }\end{array}$ & & & & $\begin{array}{l}\text { Monthly means used } \\
\text { in calculations } \\
\text { Discharge-weighted } \\
\text { means }\end{array}$ & $\begin{array}{l}\text { Trends: SMK, partial } \\
\text { MK with different } \\
\text { covariates (rainfall, T, } \\
\text { discharge) } \\
\text { Slope: not given, } \\
\text { probably Sen }\end{array}$ \\
\hline [26] & Not mentioned & $\begin{array}{l}\text { >1998, TOC HTC (ISO 8245) } \\
\text { Correlations TOC-COD } \\
\text { established, not given and not } \\
\text { used }\end{array}$ & $\begin{array}{l}\text { Data for [COD] } \\
\text { in five streams } \\
\text { shown } \\
\text { Data available in } \\
\text { internet } \\
\end{array}$ & & $\begin{array}{l}\mathrm{COD} \\
\left(\mathrm{KMnO}_{4}\right) \\
(\mathrm{ISO} \\
15705)\end{array}$ & & $\begin{array}{l}\text { Probably all } \\
\text { measured values } \\
\text { used in calculations }\end{array}$ & $\begin{array}{l}\text { Trends: MK (for COD } \\
\text { only) }\end{array}$ \\
\hline [27] & Not mentioned & TOC, see [39] & No & & & & $\begin{array}{l}\text { Biannual values, } \\
\text { treated by } \\
\text { geographical zones }\end{array}$ & Trends: SMK \\
\hline
\end{tabular}


Table 2. Cont

\begin{tabular}{|c|c|c|c|c|c|c|c|c|}
\hline Ref. & Filtration & $\begin{array}{l}\text { OC quantification } \\
\text { method }\end{array}$ & $\begin{array}{l}\text { Original data } \\
\text { plotted? }\end{array}$ & $\begin{array}{l}\text { OC range } \\
/ \mathrm{mg} \mathrm{C} \mathrm{L}^{-1} \\
\end{array}$ & $\begin{array}{l}\text { Types of } \\
\text { OC }\end{array}$ & Lake sampling & Data transformation & Statistical treatment \\
\hline [28] & $\begin{array}{l}\text { Filtered } \\
\text { (precombusted } \\
\text { GF/C filters, } \\
\text { Whatman) }\end{array}$ & $\begin{array}{l}\text { DOC: HTC (Shimadzu 5000A) } \\
\text { POC: CHN elemental analyzer } \\
\text { (Carlo Erba) }\end{array}$ & $\begin{array}{l}\text { Data for }[\mathrm{DOC}] \\
\text { and }[\mathrm{POC}] \\
\text { shown }\end{array}$ & & & $\begin{array}{l}\text { One sample at the deepest point } \\
\text { Epilimnion: sampler that collects } \\
\text { 5-L integrated samples } \\
\text { Hypolimnion: thermocline ( } 50 \mathrm{~m} \\
\text { depth) and at } 50 \mathrm{~m} \text { intervals } \\
\text { down to the bottom; integrated } \\
\text { sample by pooling volumes of } \\
\text { each sample proportional to the } \\
\text { thickness of the layer }\end{array}$ & & Trends: MK \\
\hline [29] & $\begin{array}{l}<1984, \\
\text { unfiltered in } \\
\text { Agden, } \\
\text { Broomhead, } \\
\text { Langsett but A } \\
\text { corrected: } \\
\mathrm{A}_{\text {true }}=1.06+ \\
0.63 \mathrm{~A}_{\text {apparent }} \\
{[80]}\end{array}$ & $\begin{array}{l}\text { Not measured } \\
{[\mathrm{DOC}]=0.044 * \text { Hazen }+3.89} \\
(\mathrm{r} 2=0.93, p<0.001 ; 181 \\
\text { water samples, 2005; [81]) } \\
\text { 1979-1989, Agden, Broomhead, } \\
\text { Langsett, Keighley Moor: } \\
\text { A (400 nm) } \\
\text { Hazen }=11.77 \times \mathrm{A}_{400} \\
\text { established in Broomhead }[80]\end{array}$ & No & & $\begin{array}{l}\text { Color } \\
\text { (Hazen } \\
\text { units) } \\
\text { A (400 nm) }\end{array}$ & & $\begin{array}{l}\text { Monthly and annual } \\
\text { means }\end{array}$ & $\begin{array}{l}\text { Trends: MK (annual } \\
\text { observations); SMK } \\
\text { (monthly } \\
\text { observations) } \\
\text { Slope: Sen }\end{array}$ \\
\hline [32] & Not mentioned & DOC, not mentioned & No & & & & $\begin{array}{l}\text { Mean monthly } \\
\text { concentrations }\end{array}$ & $\begin{array}{l}\text { Trends: SMK } \\
\text { Slope: Sen }\end{array}$ \\
\hline [33] & Not mentioned & DOC, not mentioned & $\begin{array}{l}\text { Yearly [DOC] } \\
\text { median values } \\
\text { vs. time shown } \\
\text { for one system }\end{array}$ & & & & $\begin{array}{l}\text { For one system: } \\
\text { yearly median } \\
\text { values; normalised } \\
\text { by log } \\
\text { transformation }\end{array}$ & $\begin{array}{l}\text { Trends: Spearman rank } \\
\text { correlationstrends }\end{array}$ \\
\hline
\end{tabular}


Table 2. Cont

\begin{tabular}{|c|c|c|c|c|c|c|c|c|}
\hline Ref. & Filtration & $\begin{array}{l}\text { OC quantification } \\
\text { method }\end{array}$ & $\begin{array}{l}\text { Original data } \\
\text { plotted? }\end{array}$ & $\begin{array}{l}\text { OC range } \\
/ \mathrm{mg} \mathrm{C} \mathrm{L}^{-1}\end{array}$ & $\begin{array}{l}\text { Types of } \\
\text { OC }\end{array}$ & Lake sampling & Data transformation & Statistical treatment \\
\hline [34] & Not mentioned & DOC, references given & $\begin{array}{l}\text { Annual [DOC] } \\
\text { Z-scores } v s . \\
\text { time shown } \\
\text { Lakes in the same } \\
\text { zone grouped }\end{array}$ & $\begin{array}{l}\text { Dorset: } \\
1.8-5.1 \\
\text { ELA: } 3.0-6.7 \\
\text { TLW: } 3.6-4.8 \\
\text { NS: } 2.1-16.2\end{array}$ & & $\begin{array}{l}\text { When }[\mathrm{DOC}] \text { measured for } \\
\text { different thermal layers, } \\
\text { whole-lake }[\mathrm{DOC}] \text { calculated } \\
\text { by adding up total OC mass in } \\
\text { each layer and dividing by lake } \\
\text { volume; no information about } \\
\text { how many lakes in this } \\
\text { situation }\end{array}$ & $\begin{array}{l}\text { 1- Mean annual ice- } \\
\text { free values } \\
2-\mathrm{Z} \text {-scores (21-yr } \\
\text { mean used) } \\
\text { 3- Trends calculated } \\
\text { by zones after } \\
\text { combination of all } \\
\text { lakes within a zone } \\
\text { and within } \\
\text { temporally coherent } \\
\text { zones }\end{array}$ & $\begin{array}{l}\text { Regional and global } \\
\text { temporal coherence: } \\
\text { Pearson's correlation } \\
\text { coefficient } \\
\text { Trends: visual } \\
\text { Slope: LR }\end{array}$ \\
\hline [36] & $\begin{array}{l}\text { Unfiltered } \\
\text { samples } \\
{[\mathrm{DOC}]=} \\
{[\mathrm{TOC}] \text { since }} \\
{[\mathrm{POC}]<5 \%} \\
{[\mathrm{DOC}][82]} \\
\text { and "sample } \\
\text { inlets of TOC } \\
\text { analyzers } \\
\text { exclude most } \\
\text { particles" }\end{array}$ & $\begin{array}{l}\text { 1993-1997: HTC Dohrmann } \\
\text { Carbon Analyzer } \\
\text { 1998-2004: Shimadzu TOC5000 } \\
\text { 2005-2007: Tekmar-Dohrman } \\
\text { Apollo 9000 }\end{array}$ & $\begin{array}{c}{[\mathrm{DOC}] \text { vs. time }} \\
\text { data shown }\end{array}$ & $\begin{array}{l}\text { L: } 18.8, \\
\text { PB: } 20.2 \\
\text { (mean } \\
\text { discharge- } \\
\text { weighted } \\
\text { [TOC]) }\end{array}$ & & & $\begin{array}{l}\text { Two calculations: one } \\
\text { based on weekly } \\
\text { samples and one on } \\
\text { annual discharge-- } \\
\text { weighted mean } \\
\text { concentrations } \\
\text { (based on a Nov- } \\
\text { Oct water year) }\end{array}$ & Trends: LR \\
\hline [37] & $\begin{array}{c}\text { Unfiltered } \\
\text { samples }\end{array}$ & $\begin{array}{l}\text { Not measured } \\
\text { "We refer to COD as the DOM } \\
\text { concentrations" } \\
\text { DOC/TOC correlations in } \\
{[46,65] \text { shown }}\end{array}$ & $\begin{array}{l}\text { Median "DOM } \\
\text { (measured as } \\
\text { COD)" vs. time } \\
\text { shown }\end{array}$ & $\begin{array}{l}\text { Median } \\
\text { reservoirs: } \\
2.1-6.2 \\
\text { Median } \\
\text { streams: } \\
3.4-9\end{array}$ & $\begin{array}{l}\mathrm{COD} \\
\qquad\left(\mathrm{KMnO}_{4}\right)\end{array}$ & No information & $\begin{array}{l}\text { Monthly } \\
\text { concentrations used } \\
\text { in calculations } \\
\text { (probably original } \\
\text { data since median } \\
\text { sampling period: } 34 \\
\text { days) }\end{array}$ & $\begin{array}{l}\text { Trends: SMK } \\
\text { Slope: Sen }\end{array}$ \\
\hline
\end{tabular}


Table 2. Cont

\begin{tabular}{|c|c|c|c|c|c|c|c|c|}
\hline Ref. & Filtration & $\begin{array}{l}\text { OC quantification } \\
\text { method }\end{array}$ & $\begin{array}{l}\text { Original data } \\
\text { plotted? }\end{array}$ & $\begin{array}{l}\text { OC range }{ }^{a} \\
/ \mathrm{mg} \mathrm{C} \mathrm{L}^{-1} \\
\end{array}$ & $\begin{array}{l}\text { Types of } \\
\text { OC }\end{array}$ & Lake sampling & Data transformation & Statistical treatment \\
\hline [38] & $\begin{array}{l}\text { Not } \\
\text { mentioned } \\
\text { but probably } \\
\text { unfiltered } \\
\text { since TOC } \\
\text { acronym is } \\
\text { used in the } \\
\text { paper }\end{array}$ & $\begin{array}{l}\text { UV-persulfate oxidation or HTC } \\
\text { Missing data } 1978-1991 \\
\text { obtained from COD: } \\
{[\text { TOC }]=1.1218+} \\
0.6435 \times \text { COD }\left(r^{2}=0.93 ;\right. \\
\text { period and number of points } \\
\text { used not given })\end{array}$ & $\begin{array}{l}\text { Mean annual } \\
\text { [TOC] vs. time } \\
\text { shown for all } \\
\text { systems } \\
\text { Probably wrong } \\
\text { units }\end{array}$ & & $\begin{array}{l}\mathrm{COD} \\
\left(\mathrm{KMnO}_{4}\right)\end{array}$ & & $\begin{array}{l}\text { Mean annual and } \\
\text { seasonal [TOC] } \\
\text { calculated by } \\
\text { averaging weekly or } \\
\text { biweekly values } \\
\text { Missing winter data } \\
\text { interpolated for } \\
\text { annual mean } \\
\text { calculation }\end{array}$ & Trends: SMK \\
\hline [39] & $\begin{array}{l}\text { Unfiltered } \\
{[\mathrm{TOC}]=} \\
{[\mathrm{DOC}]} \\
\text { based on } \\
\text { "our } \\
\text { experience } \\
\text { in these } \\
\text { waters } \\
\text { showed that } \\
\text { POM }<5 \% "\end{array}$ & $\begin{array}{l}<1994 \text {, UV-persulfate wet } \\
\text { oxidation } \\
>1995 \text { : HTC (Shimadzu) } \\
\text { Non HTC values corrected } \\
\text { according to [83] }\end{array}$ & $\begin{array}{l}{[\mathrm{TOC}] \text { vs. time }} \\
\text { data shown }\end{array}$ & $5.4-10.0$ & & & $\begin{array}{l}\text { Trends: weekly data } \\
\text { Slope: monthly } \\
\text { values }\end{array}$ & $\begin{array}{l}\text { Trends: SMK } \\
\text { Slope: not mentioned }\end{array}$ \\
\hline [40] & $\begin{array}{l}\text { Filtered } \\
\qquad(0.45 \mu \mathrm{m})\end{array}$ & DOC, no information & $\begin{array}{l}\text { All stream data } \\
\text { plotted }\end{array}$ & $\begin{array}{l}\text { Site means: } \\
2.19-11.31\end{array}$ & & & $\begin{array}{l}\text { Trends: probably } \\
\text { measured [DOC] }\end{array}$ & $\begin{array}{l}\text { LR }(95 \%) \\
\text { Periodicity of the mean } \\
\text { monthly }[\mathrm{DOC}] \text { for } \\
\text { each data set was } \\
\text { determined by } \\
\text { deconstructing the } \\
\text { time series using a } \\
\text { Discrete Fourier } \\
\text { Transform }\end{array}$ \\
\hline
\end{tabular}


Table 2. Cont

\begin{tabular}{|c|c|c|c|c|c|c|c|c|}
\hline Ref. & Filtration & $\begin{array}{l}\text { OC quantification } \\
\text { method }\end{array}$ & $\begin{array}{l}\text { Original data } \\
\text { plotted? }\end{array}$ & $\begin{array}{l}\text { OC range }{ }^{a} \\
/ \mathrm{mg} \mathrm{C} \mathrm{L}^{-1}\end{array}$ & $\begin{array}{l}\text { Types of } \\
\text { OC }\end{array}$ & Lake sampling & Data transformation & Statistical treatment \\
\hline [43] & Not mentioned & $\begin{array}{l}\text { Oxidation: UV in acidic } \\
\text { persulfate media; colorimetry } \\
\text { with phenolphthalein } \\
\text { Same method as in [56] }\end{array}$ & $\begin{array}{l}\text { Annual volume- } \\
\text { weighted } \\
\text { monthly } \\
\text { averaged } \\
\text { [DOC] and Z- } \\
\text { scores } v \text { s. time } \\
\text { shown }\end{array}$ & $\begin{array}{l}\text { Average } \\
\text { annual: } \\
2.3-10.7\end{array}$ & & & $\begin{array}{l}\text { Monthly volume- } \\
\text { weighted [DOC] } \\
\text { calculated by } \\
\text { dividing total mass } \\
\text { exported by total } \\
\text { discharge } \\
\text { Z-scores (22-yr } \\
\text { mean used) }\end{array}$ & $\begin{array}{l}\text { Trends: partial MK } \\
\text { with monthly } \\
\text { discharge as covariate } \\
\text { Comparison with } \\
\text { average [DOC] } 5 \text { first } \\
\text { years (1980-1984) }\end{array}$ \\
\hline [44] & See [43] & See $[43]$ & $\begin{array}{l}\text { Same as in [43] } \\
\text { presented } \\
\text { otherwise }\end{array}$ & See [43] & & & $\begin{array}{l}\text { Annual average and } \\
\text { annual volume-- } \\
\text { weighted annual } \\
\text { [DOC] }\end{array}$ & $\begin{array}{l}\text { Trends: MK } \\
\text { Slope: Sen }\end{array}$ \\
\hline [45] & Not mentioned & Same as [43] & No & $\begin{array}{l}\text { Average } \\
\text { annual } \\
\text { volume- } \\
\text { weighted: } \\
9.8 \\
\end{array}$ & & & $\begin{array}{l}\text { Annual volume-- } \\
\text { weighted [DOC]; } \\
\text { see }[43]\end{array}$ & Same as [43] \\
\hline [46] & $\begin{array}{l}\text { [TOC], COD: } \\
\text { unfiltered } \\
\text { samples } \\
\text { since "differ } \\
\text { by }<10 \% \text {, } \\
\text { and usually } \\
<5 \% \text { ([84- } \\
86]) \text { " } \\
\text { A: filtered } \\
(0.45 \mu \mathrm{m})\end{array}$ & $\begin{array}{l}\text { TOC, oxidative combustion (no } \\
\text { details given) } \\
\text { COD and A/TOC correlations } \\
\text { shown but not used: } \\
{[\mathrm{TOC}]=0.51+0.84 \times \mathrm{COD}} \\
\left(\mathrm{r}^{2}=0.88\right) \\
{[\mathrm{TOC}]=3.4+2.1 \times \mathrm{A}} \\
\left(\mathrm{r}^{2}=0.67\right)\end{array}$ & $\begin{array}{l}\text { One LOESS of } \\
\text { median Z- } \\
\text { scores shown }\end{array}$ & $\begin{array}{l}\text { Mean TOC } \\
\text { range: } \\
2.4-17.1\end{array}$ & $\begin{array}{l}\text { A }(420 \mathrm{~nm} \text {, } \\
5 \mathrm{~cm} \\
\text { cuvette }) \\
\mathrm{COD} \\
\left(\mathrm{KMnO}_{4}\right)\end{array}$ & & $\begin{array}{l}\text { One median (all } \\
\text { systems) Z-score } \\
\text { for LOWESS (no } \\
\text { details about } \\
\text { calculation of Z- } \\
\text { scores) }\end{array}$ & $\begin{array}{l}\text { Slope: Sen but no } \\
\text { values given } \\
\text { LOWESS smoothing } \\
\text { "for illustrative } \\
\text { purposes", span = } \\
0.75\end{array}$ \\
\hline
\end{tabular}


Table 2. Cont

\begin{tabular}{|c|c|c|c|c|c|c|c|c|}
\hline Ref. & Filtration & $\begin{array}{l}\text { OC quantification } \\
\text { method }\end{array}$ & $\begin{array}{l}\text { Original data } \\
\text { plotted? }\end{array}$ & $\begin{array}{l}\text { OC range }^{\text {a }} \\
/ \mathrm{mg} \mathrm{C} \mathrm{L}^{-1} \\
\end{array}$ & $\begin{array}{l}\text { Types of } \\
\text { OC }\end{array}$ & Lake sampling & Data transformation & Statistical treatment \\
\hline [47] & No information & DOC, no information & $\begin{array}{l}\text { Lake outflow and } \\
\text { catchment outflow } \\
\text { [DOC] vs. time } \\
\text { data shown: }\end{array}$ & $\begin{array}{l}17 \text { ("the water } \\
\text { are humic") }\end{array}$ & & Sampling point not clear & $\begin{array}{l}\text { Probably annual } \\
\text { average used }\end{array}$ & $\begin{array}{l}\text { Trends: MK } \\
\text { Slope: Sen }\end{array}$ \\
\hline [49] & $\begin{array}{l}\text { Unfiltered but } \\
\text { Dorset samples: } \\
80 \mu \mathrm{m} \text { mesh }\end{array}$ & $\begin{array}{l}\text { Same method as in [43- } \\
45,56]\end{array}$ & $\begin{array}{l}\text { Data for all lakes } \\
\text { plotted but } \\
\text { probably all are } \\
\text { annual mean } \\
\text { [DOC] } v \text { s. time }\end{array}$ & $\leq 6$ & & $\begin{array}{l}\text { Composite samples taken either } \\
\text { through the epilimnion and } \\
\text { metalimnion or volume-- } \\
\text { weighted samples accounting } \\
\text { for } \\
\text { bathymetry taken through the } \\
\text { whole water column }\end{array}$ & $\begin{array}{l}\text { Annual ice-free } \\
\text { season averages }\end{array}$ & Trends: MK \\
\hline$[50]$ & $\begin{array}{l}\text { Unfiltered } \\
\text { samples } \\
\text { [TOC] }=[\mathrm{DOC}] \\
\text { because } \\
\text { "differences } \\
\text { between TOC } \\
\text { and DOC are } \\
\text { very small }[86] "\end{array}$ & $\begin{array}{l}\text { Not measured } \\
\text { Correlation from [87]: } \\
{[\mathrm{TOC}]=0.675 \times \mathrm{COD}+} \\
1.94\end{array}$ & $\begin{array}{l}\text { [TOC] } v s . \text { time data } \\
\text { shown }\end{array}$ & & $\begin{array}{l}\mathrm{COD} \\
\left(\mathrm{KMnO}_{4}\right)\end{array}$ & & $\begin{array}{l}\text { Concentrations flow } \\
\text { adjusted using rank } \\
\text { correlation between } \\
\text { mean monthly } \\
\text { concentrations and } \\
\text { mean monthly flows }\end{array}$ & $\begin{array}{l}\text { Trends: "multivariate } \\
\text { extension" of MK, } \\
\text { first for each month } \\
\text { and then combined } 30 \\
\text { point moving average }\end{array}$ \\
\hline [51] & No information & DOC, no information & No & & & No information & $\begin{array}{l}\text { "Sites with median } \\
{[\mathrm{DOC}]<1 \mathrm{mg} \mathrm{L}^{-1}} \\
\text { excluded" }\end{array}$ & $\begin{array}{l}\text { Trends: MK } \\
\text { Slope: Sen }\end{array}$ \\
\hline [41] & $\begin{array}{l}\text { Filtered }(0.45 \mu \mathrm{m} \\
\text { polycarbonate } \\
\text { membrane } \\
\text { filters })\end{array}$ & $\begin{array}{l}\text { DOC: "OC analyzer by } \\
\text { oxidation" }\end{array}$ & $\begin{array}{l}{[\mathrm{DOC}] \text { vs. time data }} \\
\text { shown }\end{array}$ & $\begin{array}{l}\text { Mean: } \\
\text { B10: 7.3; } \\
\text { B11: } 11.4\end{array}$ & & & & $\begin{array}{l}\text { No mathematical } \\
\text { treatment }\end{array}$ \\
\hline
\end{tabular}


Table 2. Cont

\begin{tabular}{|c|c|c|c|c|c|c|c|c|}
\hline Ref. & Filtration & $\begin{array}{l}\text { OC quantification } \\
\text { method }\end{array}$ & $\begin{array}{l}\text { Original data } \\
\text { plotted? }\end{array}$ & $\begin{array}{l}\text { OC range }{ }^{a} \\
/ \mathrm{mg} \mathrm{C} \mathrm{L}^{-1}\end{array}$ & $\begin{array}{l}\text { Types of } \\
\text { OC }\end{array}$ & Lake sampling & Data transformation & Statistical treatment \\
\hline [52] & $\begin{array}{l}\text { Unfiltered } \\
\begin{array}{l}{[\mathrm{DOC}]=[\mathrm{TOC}]} \\
\text { because "[TOC }] \\
=90 \%-95 \% \\
{[\mathrm{DOC}]: \text { in these }} \\
\text { catchments" }\end{array}\end{array}$ & TOC, method not given & $\begin{array}{c}\text { Weekly }[\text { TOC] } v s . \\
\text { time data shown }\end{array}$ & $\begin{array}{l}\text { Mean annual: } \\
11.6(\mathrm{~L}), \\
5.3(\mathrm{~B}) \\
4.9(\mathrm{~S})\end{array}$ & & & $\begin{array}{l}\text { Samples with } \\
{[\text { TOC] }>18 \text { not }} \\
\text { included } \\
\text { Annual mean values } \\
\text { weighted by month }\end{array}$ & $\begin{array}{l}\text { Trends: MK, SMK } \\
\text { Slope: Sen }\end{array}$ \\
\hline [53] & Not mentioned & $\begin{array}{l}\text { Methods of analysis vary } \\
\text { between regions and } \\
\text { over the years; none } \\
\text { detailed } \\
\text { When [DOC] not } \\
\text { available: } \\
\text { [DOC] }=0.379 \times \\
\text { Color }{ }^{0.83}\left(n=477, \mathrm{r}^{2}=\right. \\
0.72 \text {; from } 44 \text { sites, used } \\
\text { in } 2 \text { sites }) \\
{[\text { DOC }]=10.09 \times} \\
\log _{\mathrm{e}}(\mathrm{COD})^{2}-7.19(n= \\
489,\left(\mathrm{r}^{2}=0.47, \text { used in }\right. \\
42 \text { sites })\end{array}$ & $\begin{array}{l}\text { 6-year moving } \\
\text { average values } v s . \\
\text { time shown for } \\
\text { some systems }\end{array}$ & & $\begin{array}{l}\text { Color } \\
\text { (Hazen } \\
\text { units) } \\
\text { COD }\end{array}$ & No information & $\begin{array}{l}\text { All records } \\
\text { "corrected to a } \\
\text { monthly time step" }\end{array}$ & $\begin{array}{l}\text { Trends: SMK } \\
\text { Moving averages } \\
\text { shown in two figures } \\
\text { but not commented }\end{array}$ \\
\hline [55] & No information & DOC, no information & No & & & $\begin{array}{l}\text { Lake water collected at the lake } \\
\text { outlet }\end{array}$ & $\begin{array}{l}\text { Months were chosen } \\
\text { as seasons }\end{array}$ & $\begin{array}{l}\text { Trends: SMK } \\
\text { Trend test applied to } \\
\text { residuals of a flow- } \\
\text { concentration model } \\
\text { (hyperbolic or log } \\
\text { regression fit) }\end{array}$ \\
\hline
\end{tabular}


Table 2. Cont

\begin{tabular}{|c|c|c|c|c|c|c|c|c|}
\hline Ref. & Filtration & $\begin{array}{l}\text { OC quantification } \\
\text { method }\end{array}$ & $\begin{array}{l}\text { Original data } \\
\text { plotted? }\end{array}$ & $\begin{array}{l}\text { OC range }{ }^{\mathrm{a}} \\
/ \mathrm{mg} \mathrm{C} \mathrm{L}^{-1}\end{array}$ & $\begin{array}{l}\text { Types of } \\
\text { OC }\end{array}$ & Lake sampling & Data transformation & Statistical treatment \\
\hline [15] & No information & DOC, no information & $\begin{array}{l}\text { Median [DOC] (for } \\
10 \text { lakes and } 8 \\
\text { streams) vs. time } \\
\text { shown }\end{array}$ & & & No information & & $\begin{array}{l}\text { Comparison median } \\
\text { [DOC] } 5 \text { first years } \\
\text { with median [DOC] } \\
\text { last } 5 \text { years (Mann- } \\
\text { Whitney test) }\end{array}$ \\
\hline [48] & $\begin{array}{l}\text { Unfiltered } \\
\text { samples } \\
{[\mathrm{DOC}]=[\mathrm{TOC}]} \\
\text { since }[\mathrm{DOC}]= \\
94 \%[\mathrm{TOC}] \text { in } \\
\text { Finnish lakes } \\
{[86]}\end{array}$ & $\begin{array}{l}<1990 \text { : COD used to } \\
\text { reconstruct [TOC], no } \\
\text { correlation given } \\
>1990: \text { UV persulfate } \\
\text { oxidation or HTC }\end{array}$ & All lake data plotted & $\begin{array}{l}\text { Mean lake } \\
\text { value range: } \\
1.5-11.3\end{array}$ & $\begin{array}{l}\mathrm{COD} \\
\qquad\left(\mathrm{KMnO}_{4}\right)\end{array}$ & Middle of the lake, $1 \mathrm{~m}$ depth & $\begin{array}{l}\text { Trends in annual and } \\
\text { individual months } \\
\text { evaluated }\end{array}$ & $\begin{array}{l}\text { Trends: SMK } \\
\text { Slope: Sen }\end{array}$ \\
\hline [31] & No information & No information & $\begin{array}{l}\text { [DOC] } v s . \text { time data } \\
\text { shown }\end{array}$ & & & & & $\begin{array}{l}\text { No mathematical } \\
\text { treatment }\end{array}$ \\
\hline [56] & $\begin{array}{l}\text { Unfiltered, } \\
\text { prefiltered: } \\
80 \mu \mathrm{m} \text { polyester } \\
\text { mesh }\end{array}$ & $\begin{array}{l}\text { DOC: oxidation: UV in } \\
\text { acidic persulfate media; } \\
\text { colorimetry with } \\
\text { phenolphthalein }\end{array}$ & $\begin{array}{l}\text { 3-year running } \\
\text { mean normalised } \\
\text { with long-term } \\
\text { mean } \\
\end{array}$ & $1.80-5.23$ & & No information & & $\begin{array}{l}\text { Calculation of 3-year } \\
\text { running means } \\
\text { normalised with } \\
\text { long-term mean } \\
\end{array}$ \\
\hline [16] & No information & DOC, no information & All data plotted & & & No information & & $\begin{array}{l}\text { Trends: SMK } \\
\text { Slope: Sen }\end{array}$ \\
\hline [57] & $\begin{array}{l}\text { Filtered (glass } \\
\text { fibber filters; no } \\
\text { size given) }\end{array}$ & $\begin{array}{l}\text { 1988-1993: persulfate } \\
\text { digestion (ASTRO 2001) } \\
\text { 1994-2003: HTC } \\
\text { (Shimadzu 5000) } \\
\text { Intercalibration for 1 year }\end{array}$ & $\begin{array}{l}\text { Mean annual [DOC] } \\
\text { vs. time plotted for } \\
3 \text { stations }\end{array}$ & $\approx 4$ & & & & Trends: probably LR \\
\hline
\end{tabular}


Table 2. Cont

\begin{tabular}{|c|c|c|c|c|c|c|c|c|}
\hline Ref. & Filtration & $\begin{array}{l}\text { OC quantification } \\
\text { method }\end{array}$ & $\begin{array}{l}\text { Original data } \\
\text { plotted? }\end{array}$ & $\begin{array}{l}\text { OC range }{ }^{a} \\
/ \mathrm{mg} \mathrm{C} \mathrm{L}^{-1}\end{array}$ & $\begin{array}{l}\text { Types of } \\
\text { OC }\end{array}$ & Lake sampling & Data transformation & Statistical treatment \\
\hline [23] & $\begin{array}{l}\text { GFC filters } \\
\text { (filtered in the } \\
\text { field) }\end{array}$ & $\begin{array}{l}\text { HTC (TOCsin II Aqueous } \\
\text { Carbon Analyzer) }\end{array}$ & $\begin{array}{l}\text { [DOC] vs. time data } \\
\text { shown for } 3 \\
\text { streams }\end{array}$ & $\begin{array}{l}\text { Moorlands } \\
\text { and forest: } \\
1.5 \text { (up to } 14 \\
\text { in small } \\
\text { streams) }\end{array}$ & & & $\begin{array}{l}\text { Raw data and } \\
\text { residuals after flow } \\
\text { and season filter }\end{array}$ & $\begin{array}{r}\text { Slope: LR, SMK } \\
\text { (probably Sen) }\end{array}$ \\
\hline$[18]^{\mathrm{c}}$ & $\begin{array}{l}\text { No information, } \\
\text { probably in [9] }\end{array}$ & $\begin{array}{l}\text { No information, probably } \\
\text { in [9] }\end{array}$ & No & & & No information & & $\begin{array}{l}\text { Trends: calculation of } \\
\text { confidence limits } \\
\text { about the median } \\
\text { value in the slope } \\
\text { distribution and } \\
\text { testing for zero } \\
\text { inclusion } \\
\text { Slope: LR }\end{array}$ \\
\hline$[58]$ & Not mentioned & $\begin{array}{l}\text { TOC, oxidation to } \mathrm{CO}_{2} \\
\text { and IR detection (no } \\
\text { details on type of } \\
\text { oxidation) }\end{array}$ & No & & & & $\begin{array}{l}\text { Trends studied in } \\
\text { March, May, } \\
\text { August and October }\end{array}$ & Trends: SMK \\
\hline [59] & Not mentioned & $\begin{array}{l}\text { >1986: UV-persulfate } \\
\text { oxidation (Astro 1859) } \\
>1996: \text { HTC (Astro 2100) } \\
\text { >1999: HTC (Shimadzu } \\
\text { 5000) }\end{array}$ & $\begin{array}{l}\text { Color, }[\mathrm{COD}] \text { and } \\
{[\mathrm{DOC}] \text { vs. time }} \\
\text { data shown for a } \\
\text { water treatment } \\
\text { plant inlet }\end{array}$ & & $\begin{array}{l}\text { Color } \\
\text { (Pt units) } \\
\text { COD } \\
\left(\mathrm{KMnO}_{4}\right)\end{array}$ & $40 \mathrm{~m}$ depth & & $\begin{array}{l}\text { Apparently, no } \\
\text { mathematical } \\
\text { treatment }\end{array}$ \\
\hline [47] & No information & DOC, no information & $\begin{array}{l}\text { Probably monthly } \\
\text { mean data, not } \\
\text { original data, for } \\
\text { Great Dun Fell, } \\
\text { Upper Hafron, } \\
\text { Warkworth }\end{array}$ & & & No information & $\begin{array}{l}\text { All monitoring } \\
\text { records converted to } \\
\text { a monthly time step; } \\
\text { then annual average } \\
\text { [DOC] }\end{array}$ & $\begin{array}{l}\text { Trends: SMK } \\
\text { Slope: probably Sen }\end{array}$ \\
\hline
\end{tabular}


Table 2. Cont

\begin{tabular}{|c|c|c|c|c|c|c|c|c|}
\hline Ref. & Filtration & $\begin{array}{l}\text { OC quantification } \\
\text { method }\end{array}$ & $\begin{array}{l}\text { Original data } \\
\text { plotted? }\end{array}$ & $\begin{array}{l}\text { OC range }{ }^{a} \\
/ \mathbf{m g ~ C ~ L}^{-1}\end{array}$ & $\begin{array}{l}\text { Types of } \\
\text { OC }\end{array}$ & Lake sampling & Data transformation & Statistical treatment \\
\hline$[30]$ & No information & DOC, no information & $\begin{array}{l}\text { Weekly }[\mathrm{DOC}] v s . \\
\text { time data shown } \\
\text { for the Moor } \\
\text { House catchment } \\
\text { outlet }\end{array}$ & & & & & Trends: SMK \\
\hline$[60]$ & See $[61]$ & See $[61]$ & $\begin{array}{l}\text { Monthly average } \\
\text { color vs. time } \\
\text { shown for Coquet } \\
\text { and Tees (in this } \\
\text { case, same figure } \\
\text { as in [61]). } \\
\text { Annual median data } \\
\text { also shown }\end{array}$ & & See $[61]$ & & & Trends: SMK \\
\hline$[62]$ & No information & DOC, no information & $\begin{array}{l}\text { [DOC] } v s . \text { time data } \\
\text { shown for Big } \\
\text { Moose }\end{array}$ & $\begin{array}{c}38 \text { lakes < } \\
500 \mu \mathrm{M}\end{array}$ & & No information & & Trends: SMK \\
\hline [64] & No information & TOC, no information & No & & & $\begin{array}{l}\text { Samples taken either from the } \\
\text { middle of the lake ( } 1 \mathrm{~m} \text { depth) } \\
\text { or at the outlet }\end{array}$ & & $\begin{array}{l}\text { Trends: MK } \\
\text { Slope: LR }\end{array}$ \\
\hline [65] & Not mentioned & $\begin{array}{l}\text { Not measured } \\
\text { Calibration DOC-COD: } \\
{[\mathrm{DOC}]=1.4+} \\
0.67 \times \mathrm{COD}(n=235, \\
\left.\mathrm{r}^{2}=0.88, p<0.001\right) \\
\text { from measurements } \\
1995-1998 \text { but not used; } \\
\text { trend results given in } \\
\text { COD values }\end{array}$ & $\begin{array}{l}\text { Monthly mean COD } \\
\text { vs. time shown }\end{array}$ & & $\begin{array}{l}\mathrm{COD} \\
\left(\mathrm{KMnO}_{4}\right)\end{array}$ & & $\begin{array}{l}\text { SMK applied to } \\
\text { "monthly average } \\
\text { blocks of data" }\end{array}$ & $\begin{array}{l}\text { Trends: SMK } \\
\text { Slope: Sen }\end{array}$ \\
\hline
\end{tabular}


Table 2. Cont

\begin{tabular}{|c|c|c|c|c|c|c|c|c|}
\hline Ref. & Filtration & $\begin{array}{l}\text { OC quantification } \\
\text { method }\end{array}$ & $\begin{array}{l}\text { Original data } \\
\text { plotted? }\end{array}$ & $\begin{array}{l}\text { OC range }{ }^{a} \\
/ \mathbf{m g ~ C ~ L}^{-1}\end{array}$ & $\begin{array}{l}\text { Types of } \\
\text { OC }\end{array}$ & Lake sampling & Data transformation & Statistical treatment \\
\hline [35] & Not mentioned & $\begin{array}{l}\text { DOC: UV radiation in acid } \\
\text { persulfate media; } \\
\text { colorimetry with } \\
\text { phenolphthalein }\end{array}$ & $\begin{array}{l}\text { Average [DOC] and } \\
\text { Z-scores vs. time } \\
\text { for the ice-free } \\
\text { season shown }\end{array}$ & $\begin{array}{l}\text { Mean lake } \\
\text { value range: } \\
1.8-5.1\end{array}$ & & $\begin{array}{l}\text { Samples collected at the deepest } \\
\text { location in the lake either from } \\
\text { the upper } 5 \mathrm{~m} \text { of the water } \\
\text { column during the spring and } \\
\text { fall overturns or from the } \\
\text { entire water column during } \\
\text { stratification. In this case, } \\
\text { samples collected every } 2 \mathrm{~m} \\
\text { were volume-weighted for } \\
\text { each thermal layer and then } \\
\text { volume weighted to give a } \\
\text { single value for each sampling } \\
\text { date }\end{array}$ & $\begin{array}{l}\text { Calculation of: } \\
\text { - mean [DOC] of } \\
\text { all sampling dates } \\
\text { for each ice-free } \\
\text { season } \\
\text { - mean annual ice- } \\
\text { free [DOC] } \\
\text { standardised to Z- } \\
\text { scores (21-yr mean } \\
\text { used) }\end{array}$ & $\begin{array}{l}\text { No mathematical } \\
\text { treatment; visual } \\
\text { inspection figure }\end{array}$ \\
\hline [66] & No information & DOC, no information & No & & & & & $\begin{array}{l}\text { "Non-parametric test } \\
\text { procedures" } \\
\text { considering } \\
\text { seasonality and } \\
\text { autocorrelation } \\
\end{array}$ \\
\hline [67] & No information & DOC, no information & No & & & & $\begin{array}{l}\text { Probably original } \\
\text { (annual) data used } \\
\text { in the calculations }\end{array}$ & $\begin{array}{l}\text { Trends: SMK } \\
\text { (autocorrelation } \\
\text { considered) }\end{array}$ \\
\hline [41] & No information & $\begin{array}{l}\text { DOC, no information } \\
\text { Correlation DOC-A: } \\
{[\mathrm{DOC}]=0.58+16.4 \times \mathrm{A}} \\
\left(\mathrm{r}^{2}=0.89, n=586\right) \\
\text { for one stream (same } \\
\text { figure) but never used }\end{array}$ & $\begin{array}{l}\text { [DOC] } v \text { s. time data } \\
\text { shown only for } \\
\text { one stream in } \\
\text { Loch Ard area }\end{array}$ & $1.3-36.8$ & A $(250 \mathrm{~nm})$ & No information & & $\begin{array}{l}\text { Trends: SMK and } \\
\text { permutation based LR }\end{array}$ \\
\hline
\end{tabular}


Table 2. Cont.

\begin{tabular}{|c|c|c|c|c|c|c|c|c|}
\hline Ref. & Filtration & $\begin{array}{l}\text { OC quantification } \\
\text { method }\end{array}$ & $\begin{array}{l}\text { Original data } \\
\text { plotted? }\end{array}$ & $\begin{array}{l}\text { OC range } \\
/ \mathrm{mg} \mathrm{C} \mathrm{L}^{-1}\end{array}$ & $\begin{array}{l}\text { Types of } \\
\text { OC }\end{array}$ & Lake sampling & Data transformation & Statistical treatment \\
\hline [61] & No filtration & $\begin{array}{l}\text { Not measured } \\
\text { Calibration DOC-color: } \\
{[\mathrm{DOC}]=1.09+0.051 \times \text { Color }} \\
\text { from measurements } 20 \text { June } \\
2000 \text { but not used; trend } \\
\text { results based on color values }\end{array}$ & $\begin{array}{l}\text { Monthly average } \\
\text { water color for } \\
\text { River Tees and } \\
\text { annual average } \\
\text { color vs. time for } \\
\text { all rivers shown }\end{array}$ & & $\begin{array}{l}\text { Color } \\
\text { (Hazen } \\
\text { units) }\end{array}$ & & $\begin{array}{l}\text { Probably monthly } \\
\text { average values used }\end{array}$ & $\begin{array}{l}\text { Trends: visual } \\
\text { Slope: LR }\end{array}$ \\
\hline [14] & No information & DOC, no information & $\begin{array}{l}\text { Median [DOC] Z- } \\
\text { scores for lakes } \\
\text { and rivers } v s . \text { time } \\
\text { shown }\end{array}$ & & & No information & $\begin{array}{l}\text { Z-score calculated } \\
\text { from quarterly data } \\
\text { for lakes and } \\
\text { monthly data for } \\
\text { rivers }\end{array}$ & Trends: SMK \\
\hline [68] & - & Not measured & $\begin{array}{l}\text { Data shown for } 4 \\
\text { lakes }\end{array}$ & & $\begin{array}{l}\text { Secchi disk } \\
\text { depth }\end{array}$ & & & LR \\
\hline [69] & No information & DOC, no information & No & & & No information & & $\begin{array}{l}\text { Trends: SMK and } \\
\text { permutation based LR }\end{array}$ \\
\hline [70] & No information & DOC, no information & No & & & No information & & $\begin{array}{l}\text { Trends: SMK } \\
\text { (autocorrelation } \\
\text { considered) } \\
\text { Slope: Sen } \\
\end{array}$ \\
\hline [71] & Not mentioned & TOC, no information & No & $\begin{array}{l}\text { Colored dots } \\
\text { on a map } \\
(0.1-100 \\
\text { scale })\end{array}$ & & $\begin{array}{l}\text { Norway, Finland: sampling at } \\
\text { the outlet after autumn } \\
\text { circulation period; Sweden: } \\
\text { "sampled in the middle of the } \\
\text { lake" }\end{array}$ & $\begin{array}{l}\text { Probably original } \\
\text { (annual) data used } \\
\text { in the calculations }\end{array}$ & $\begin{array}{l}\text { Trends: MK } \\
\text { Slope: Sen }\end{array}$ \\
\hline [72] & Not mentioned & Not measured & $\begin{array}{l}\text { Color data for River } \\
\text { Lielupe and } \\
\text { [COD] data for } \\
\text { River Gauja } \\
\text { shown }\end{array}$ & & $\begin{array}{l}\text { Color } \\
\text { (Pt scale) } \\
\mathrm{COD} \\
\left(\mathrm{K}_{2} \mathrm{Cr}_{2} \mathrm{O}_{7}\right)\end{array}$ & & $\begin{array}{l}\text { Original (monthly) } \\
\text { data used in the } \\
\text { calculations }\end{array}$ & $\begin{array}{l}\text { Trends: MK, SMK } \\
\text { Slope: Sen }\end{array}$ \\
\hline
\end{tabular}


Table 2. Cont.

\begin{tabular}{|c|c|c|c|c|c|c|c|c|}
\hline Ref. & Filtration & $\begin{array}{l}\text { OC quantification } \\
\text { method }\end{array}$ & $\begin{array}{l}\text { Original data } \\
\text { plotted? }\end{array}$ & $\begin{array}{l}\text { OC range }{ }^{a} \\
/ \mathrm{mg} \mathrm{C} \mathrm{L}^{-1}\end{array}$ & $\begin{array}{l}\text { Types of } \\
\text { OC }\end{array}$ & Lake sampling & Data transformation & Statistical treatment \\
\hline [73] & Not mentioned & DOC, no information & No & $\begin{array}{l}\text { DOC range: } \\
0.53-16.70 \\
\end{array}$ & & No information & & $\begin{array}{l}\text { Trends: MK } \\
\text { Slope: LR }\end{array}$ \\
\hline [74] & Not mentioned & $\begin{array}{l}\text { DOC: "determined by } \\
\text { autoanalyzer" }\end{array}$ & $\begin{array}{l}\text { [DOC] vs. time data } \\
\text { shown for Truite } \\
\text { Rouge and Eclair } \\
\text { lakes }\end{array}$ & $\begin{array}{l}\text { Range of region' } \\
\text { means: } \\
253.1-564.5 \\
\mu \mathrm{M}\end{array}$ & & $\begin{array}{l}\text { Integrated } 0-5 \mathrm{~m} \text { lakewater } \\
\text { samples collected with a } \\
\text { sampling iron }\end{array}$ & & $\begin{array}{l}\text { Trends: MK, SMK, } \\
\text { Spearman } \\
\text { /Lettenmaier, Hirsch } \\
\text { and Slack tests } \\
\text { (autocorrelation } \\
\text { considered) } \\
\text { Slope: LR }\end{array}$ \\
\hline [75] & $\begin{array}{l}\text { Filtered } \\
\text { (precombusted } \\
\text { Whatman } \\
\text { GF/F filters) }\end{array}$ & $\begin{array}{l}\text { DOC digestion: acid persulfate } \\
\text { by autoclaving (1971-75), } \\
\text { UV irradiation (1975-85), } \\
\text { heating to } 102{ }^{\circ} \mathrm{C}(>1986) \\
\mathrm{CO}_{2} \text { measurement: GC: } \\
\text { thermal conductivity detector } \\
\text { (1971-75), specific } \\
\text { conductance after Ba } \\
\text { stripping (1976-85), IR } \\
\text { (>1986) } \\
\text { Method changes } \\
\text { intercalibrated; no details }\end{array}$ & $\begin{array}{l}\text { Mean annual [DOC] } \\
\text { vs. time shown for } \\
\text { all lakes }\end{array}$ & & & $\begin{array}{l}\text { Measurements "at several depths } \\
\text { in the water column of each } \\
\text { lake"; no information about } \\
\text { treatment of these values }\end{array}$ & & $\begin{array}{l}\text { No mathematical } \\
\text { treatment; probably } \\
\text { visual inspection of } \\
\text { figure }\end{array}$ \\
\hline [63] & Not mentioned & $\begin{array}{l}\text { DOC: UV persulfate oxidation, } \\
\mathrm{CO}_{2} \text { detection by IR }\end{array}$ & $\begin{array}{l}\text { [DOC] } v s . \text { time data } \\
\text { shown for } \\
\text { Constable and } \\
\text { Arbutus ponds }\end{array}$ & $\begin{array}{l}\text { Mean lake value } \\
\text { range: } \\
192-1132 \mu \mathrm{M}\end{array}$ & - & $\begin{array}{l}\text { Sampling at the outlet of } \\
\text { drainage lakes (15) and at the } \\
\text { surface of seepage lakes (2) }\end{array}$ & $\begin{array}{l}\text { Original (monthly) } \\
\text { data used in the } \\
\text { calculations }\end{array}$ & $\begin{array}{l}\text { Trends: SMK } \\
\text { (autocorrelation } \\
\text { considered) } \\
\text { Slope: Sen }\end{array}$ \\
\hline
\end{tabular}


Table 2. Cont

\begin{tabular}{|c|c|c|c|c|c|c|c|c|}
\hline Ref. & Filtration & $\begin{array}{l}\text { OC quantification } \\
\text { method }\end{array}$ & $\begin{array}{l}\text { Original data } \\
\text { plotted? }\end{array}$ & $\begin{array}{l}\text { OC range }^{\mathrm{a}} \\
/ \mathrm{mg} \mathrm{C} \mathrm{L}^{-1}\end{array}$ & Types of $\mathrm{OC}$ & Lake sampling & Data transformation & Statistical treatment \\
\hline [76] & $\begin{array}{l}\text { Filtered } \\
\qquad(0.45 \mu \mathrm{m})\end{array}$ & Not measured & $\begin{array}{l}\text { A (Alsterälven) and } \\
\text { color (Lake } \\
\text { Öjaren) annual } \\
\text { means vs. time } \\
\text { shown }\end{array}$ & & $\begin{array}{l}\text { Rivers: A } \\
\text { (420 nm, } 5 \\
\text { cm cuvette) } \\
\text { Lake: color } \\
\text { (Pt scale) }\end{array}$ & No information & & Visual \\
\hline [77] & $\begin{array}{l}\text { Rivers: filtered } \\
\quad(0.45 \mu \mathrm{m}) \\
\text { Lakes: no } \\
\text { information }\end{array}$ & Not measured & $\begin{array}{l}\text { A vs. time shown } \\
\text { for River } \\
\text { Botorpsström and } \\
\text { A annual means } \\
v s . \text { time for rivers } \\
\text { Botorpsström and } \\
\text { Ätran }\end{array}$ & & $\begin{array}{l}\text { Rivers: A } \\
\text { (420 nm, } 5 \\
\text { cm cuvette) } \\
\text { Lakes: color } \\
\text { (Pt scale) }\end{array}$ & No information & & Value comparison \\
\hline [78] & Not mentioned & Not measured & $\begin{array}{l}\text { Color annual means } \\
\text { vs. time for lakes } \\
\text { Oxsjön and } \\
\text { Hammardammen } \\
\text { shown }\end{array}$ & & $\begin{array}{l}\text { Color }(\mathrm{Pt} \\
\text { scale) }\end{array}$ & No information & & $\begin{array}{l}\text { Comparison of values } \\
\text { from initial and final } \\
\text { years }\end{array}$ \\
\hline [79] & $\begin{array}{l}\text { Filtered } \\
\qquad(0.45 \mu \mathrm{m} \\
\text { Millipore } \\
\text { membrane } \\
\text { filters })\end{array}$ & Not measured & $\begin{array}{l}\text { A monthly mean } \\
\text { averages and } \\
\text { 12-month running } \\
\text { means } v \text { s. time } \\
\text { shown }\end{array}$ & & A $(400 \mathrm{~nm})$ & & $\begin{array}{l}\text { Original data } \\
\text { averaged to monthly } \\
\text { values }\end{array}$ & $\begin{array}{l}\text { 12-month running } \\
\text { means }\end{array}$ \\
\hline
\end{tabular}

Note: ${ }^{a}$ All OC concentration values in the table are in $\mathrm{mg} \mathrm{C} \mathrm{L}^{-1}$ except when stated otherwise. 
Table 3. Published long-term temporal trends in organic carbon concentrations in freshwaters. ${ }^{\text {a }}$

\begin{tabular}{|c|c|c|c|c|c|c|c|c|}
\hline Ref. & $\begin{array}{l}\text { Type of } \\
\text { system }\end{array}$ & No. & $\begin{array}{l}\text { Measured } \\
\text { parameter }^{\text {b }}\end{array}$ & Temporal trend? ${ }^{c}$ & $\begin{array}{l}\text { Trend magnitude } \\
\text { / } \mathrm{mgC} \mathrm{L}^{-1} \mathbf{y}^{-1}\end{array}$ & Comments & Period & Location \\
\hline [20] & river & 1 & TOC, DOC & $\begin{array}{l}\text { DOC "decreased from around } 1000 \text { to } \\
\text { around } 500 \mu \mathrm{mol} \mathrm{L}{ }^{-1} \text { " in the late } \\
1980 \mathrm{~s} \text {, constant after 1996; no stats }\end{array}$ & - & $\begin{array}{l}\text { Relative \% of DOC and POC } \\
\text { changed }\end{array}$ & 1985-2007 & Germany \\
\hline [21] & lakes & 30 & DOC & $\begin{array}{l}\text { 22: ss increasing }(p<0.05) \\
\text { 8: no trend }\end{array}$ & $\begin{array}{l}\text { Range: } 0.01-0.14 \\
\text { (individual values given) }\end{array}$ & $\begin{array}{l}\text { Trend not related to initial } \\
\text { (1989) or mean [DOC] }\end{array}$ & 1989-2006 & $\begin{array}{l}\text { Quebec, } \\
\text { Canada }\end{array}$ \\
\hline [22] & stream & $\begin{array}{l}\text { not } \\
\text { clear }\end{array}$ & DOC & Increase, no stats & - & & 1990-2010 & $\begin{array}{l}\text { Wales, } \\
\text { United } \\
\text { Kingdom }\end{array}$ \\
\hline [24] & stream & 1 & DOC & ss increase $(p=0.023)$ & 0.02 & & $\begin{array}{l}\text { 1987-1994, } \\
1999-2009\end{array}$ & $\begin{array}{l}\text { Ontario, } \\
\text { Canada }\end{array}$ \\
\hline [26] & rivers & 11 & COD & $\begin{array}{l}5 \text { (out of } 6 \text { small rivers) N Estonia: } \\
\text { ss increase (at least } p<0.05) \\
\text { Pärnu: increase }(0.05<p<0.1) \\
4 \text { S Estonia: no trend }\end{array}$ & - & COD slopes & 1992-2007 & Estonia \\
\hline [27] & lakes & 91 & TOC & $\begin{array}{l}\text { ss increase: } \\
p>99 \%: \text { NB }(n=13)(2000-2007) \\
p \text { 95\%: NF }(n=14)(2000-2007), \\
\text { WNS }(n=45)(1983-2007,1990- \\
2007,2000-2007), \text { ENS }(n=23) \\
(2000-2007) \\
p 90 \%: \text { NF }(1983-2007) \\
\text { no trend: NF }(1990-2007), \text { ENS }(1990- \\
\text { 2007) }\end{array}$ & - & & $\begin{array}{l}\text { Newfoundland (NF), W } \\
\text { Nova Scotia (WNS): } \\
\text { 1983-2007 } \\
\text { E Nova Scotia (ENS): } \\
\text { 1990-2007 } \\
\text { New Brunswick (NB): } \\
\text { 2000-2007 }\end{array}$ & Canada \\
\hline [28] & lake & 1 & DOC, POC & $\begin{array}{l}\text { DOC: ss decrease, epilimnion and } \\
\text { hypolimnion }(p<0.0001) \\
\text { POC: no trends }\end{array}$ & - & $\begin{array}{l}\text { [DOC] halved in } 20 \mathrm{y} \text { (from } \\
119 \text { to } 57 \mu \mathrm{mol} \mathrm{L}^{-1} \text { ) but it } \\
\text { was discontinuous (peaks } \\
1996-1999 \text { ) }\end{array}$ & 1980-2007 & Switzerland \\
\hline
\end{tabular}


Table 3. Cont

\begin{tabular}{|c|c|c|c|c|c|c|c|c|}
\hline Ref. & $\begin{array}{l}\text { Type of } \\
\text { system }\end{array}$ & No. & $\begin{array}{l}\text { Measured } \\
\text { parameter }^{b}\end{array}$ & Temporal trend? ${ }^{c}$ & $\begin{array}{l}\text { Trend magnitude } \\
/ \mathrm{mgC} \mathrm{L}^{-1} \mathbf{y}^{-1}\end{array}$ & Comments & Period & Location \\
\hline [29] & streams & 6 & $\begin{array}{l}\text { DOC estimated } \\
\text { from color and } \\
\text { A }(400 \mathrm{~nm})\end{array}$ & ss increases, $p<0.001$ & $\begin{array}{l}\text { Trout Beck: } 0.06 \\
\text { L. Laithe: } 0.09 \\
\text { K. Moor: } 0.32 \\
\text { Agden: } 0.20 \\
\text { B'head: } 0.30 \\
\text { Langsett: } 0.33\end{array}$ & $\begin{array}{l}\text { Authors qualify this DOC as } \\
\text { "humic DOC" }\end{array}$ & $\begin{array}{l}\text { T’ Beck: 1993-2006 } \\
\text { L' Laithe: 1994-2006 } \\
\text { K' Moor: 1979-2006 } \\
\text { Agden, Broomhead, } \\
\text { Langsett: 1961-2006 }\end{array}$ & $\begin{array}{l}\text { United } \\
\text { Kingdom }\end{array}$ \\
\hline [32] & stream & 1 & DOC & $\begin{array}{l}\text { "DOC concentrations have not varied } \\
\text { substantially or systematically" }\end{array}$ & - & & 1988-2006 & USA \\
\hline [33] & $\begin{array}{l}\text { moorland } \\
\text { ponds }\end{array}$ & 4 & DOC & $\begin{array}{l}\text { Achterste Goorven: increase, } p<0.05 \\
\quad(n=29) \\
\text { Schaapsven: increase, } p<0.01(n=8) \\
\text { Groot Huisven, Wolfsputven: no trends }\end{array}$ & - & & 1978-2006 & Netherlands \\
\hline [34] & lakes & 55 & DOC & $\begin{array}{l}\text { Cyclic pattern: decrease, increase, } \\
\text { decrease } \\
\text { Only one region with ss increase: ELA } \\
\text { (4 lakes) }(p=0.015)\end{array}$ & ELA: 0.03 & $\begin{array}{l}\text { Synchronous within regions, } \\
\text { not synchronous across } \\
\text { regions except in Nova Scotia }\end{array}$ & $1981-2003$ & $\begin{array}{l}\text { Ontario, } \\
\text { Canada }\end{array}$ \\
\hline [36] & streams & 2 & TOC & ss increase $(p<0.001)$ & $\begin{array}{l}\text { Weekly samples: } \\
\text { Lysina: } 0.42 \text {, Pluhuv } \\
\text { Bor: } 0.43 \\
\text { Annual discharge- } \\
\text { weighted mean } \\
\text { concentrations: } \\
\text { Lysina: } 0.62 \text {, Pluhuv } \\
\text { Bor: } 0.93\end{array}$ & $\begin{array}{l}\text { Lysina: } 64 \% \text { increase, Pluhuv } \\
\text { Bor: } 65 \% \text {, taking as reference } \\
\text { mean } 1993-1994\end{array}$ & 1993-2007 & $\begin{array}{l}\text { Czech } \\
\text { Republic }\end{array}$ \\
\hline
\end{tabular}


Table 3. Cont

\begin{tabular}{|c|c|c|c|c|c|c|c|c|}
\hline Ref. & $\begin{array}{l}\text { Type of } \\
\text { system }\end{array}$ & No. & $\begin{array}{l}\text { Measured } \\
\text { parameter }^{b}\end{array}$ & Temporal trend? ${ }^{c}$ & $\begin{array}{l}\text { Trend magnitude } \\
/ \mathbf{m g C ~ L}^{-1} \mathbf{y}^{-1}\end{array}$ & Comments & Period & Location \\
\hline [37] & $\begin{array}{l}\text { reservoirs } \\
\text { streams }\end{array}$ & $\begin{array}{l}7 \\
4\end{array}$ & COD & $\begin{array}{l}\text { Reservoirs: } 5 \text { ss increase, }(p<0.001 \text {, } \\
\text { except Karmenicka: } p<0.01), 2 \text { no } \\
\text { trend } \\
\text { Streams: all ss increase, }(p<0.001 \text {, } \\
\text { except Cerna voda: } p<0.05)\end{array}$ & - & $\begin{array}{l}\text { COD slopes } \\
\text { COD increase positively } \\
\text { correlated with average }[\mathrm{COD}] \\
\left(\mathrm{R}^{2}=0.79, p<0.001\right)\end{array}$ & $\begin{array}{l}\text { reservoirs: } 1969-2006 \\
\text { streams: }(1969,1974, \\
\text { 1983)-2006 }\end{array}$ & $\begin{array}{l}\text { Czech } \\
\text { Republic }\end{array}$ \\
\hline [38] & streams & 8 & $\begin{array}{l}\text { TOC, } 1978- \\
1991 \text { estimated } \\
\text { from COD }\end{array}$ & $\begin{array}{l}7: \text { increase }(p<0.05) \\
1: \text { no trend }\end{array}$ & - & $\begin{array}{l}\text { Annual trends became detectable } \\
\text { when there was at least one } \\
\text { season with ss increase }\end{array}$ & $\begin{array}{l}\text { 2: } 1979-2006 \\
\text { 3: } 1979-1982,1996-2005 \\
\text { 3: } 1992-2006\end{array}$ & Finland \\
\hline [39] & streams & 3 & TOC & $\begin{array}{l}\text { Uncorrected. Mersey (1980-2005), } \\
\text { Moose Pit (1983-2005), Pine Marten } \\
\text { (1991-2005): no trend } \\
\text { Uncorrected. Mersey (1980-1994): } \\
\text { ss decrease ( } p=0.06), \text { Moose Pit } \\
\text { (1983-1994): ss decrease ( } p=0.05) \\
\text { Uncorrected. Mersey, Moose Pit, Pine } \\
\text { Marten (1995-2005): no trend } \\
\text { Corrected. Mersey (1980-2005): } \\
\text { ss decrease ( } p=0.04), \text { Moose Pit } \\
\text { (1983-2005): ss decrease ( } p=0.008) \text {, } \\
\text { Pine Marten (1991-2005): no trend }\end{array}$ & $\begin{array}{l}\text { Corrected (all period): } \\
\text { Mersey: }-0.1, \\
\text { Moose Pit: }-0.25 \\
\text { Uncorrected }(<1994) \text { : } \\
\text { Mersey: }-0.25, \\
\text { Moose Pit: }-0.58\end{array}$ & $\begin{array}{l}\text { Values }<1994 \text { corrected for } \\
\text { differences in OC method } \\
\text { response }\end{array}$ & $\begin{array}{l}\text { Mersey: 1980-2005 } \\
\text { Moose Pit: 1983-2005 } \\
\text { Pine Marten: } 1991-2005\end{array}$ & Canada \\
\hline$[40]^{\mathrm{e}}$ & streams & 6 & DOC & $\begin{array}{l}\text { Loch Ard (3), Allt a'Mharcaidh (1): } \\
\text { increase }(p<0.001) \\
\text { Sourhope (2): no trend }\end{array}$ & $\begin{array}{l}\text { Loch Ard, Burn 2: } 0.28 \\
\text { Loch Ard, Burn 10: } 0.22 \\
\text { Loch Ard, Burn 11: } 0.79 \\
\text { Allt a'Mharcaidh: } 0.15\end{array}$ & $\begin{array}{l}\text { Marked seasonal pattern } \\
\text { (particularly in Loch Ard), } \\
\text { with an increasing amplitude in } \\
\text { latter years }\end{array}$ & $\begin{array}{l}\text { Burn 2: 1989-2002 } \\
\text { Burn 10, 11: 1988-2003 } \\
\text { Allt a'Mharcaidh: } \\
\text { 1987-2002 } \\
\text { Sourhope: 1995-2006 }\end{array}$ & $\begin{array}{l}\text { Scotland, } \\
\text { United } \\
\text { Kingdom }\end{array}$ \\
\hline
\end{tabular}


Table 3. Cont.

\begin{tabular}{|c|c|c|c|c|c|c|c|c|}
\hline Ref. & $\begin{array}{l}\text { Type of } \\
\text { system }\end{array}$ & No. & $\begin{array}{l}\text { Measured } \\
\text { parameter }^{\text {b }}\end{array}$ & Temporal trend? ${ }^{c}$ & $\begin{array}{l}\text { Trend magnitude }{ }^{\mathbf{d}} \\
/ \mathrm{mgC} \mathrm{L}^{-1} \mathbf{y}^{-1}\end{array}$ & Comments & Period & Location \\
\hline [43] & streams & 7 & DOC & $\begin{array}{l}6 \text { wetland-dominated streams: } \\
\text { ss increase }(p<0.05) \\
1 \text { upland-dominated stream: no trend }\end{array}$ & - & $\begin{array}{l}\text { Wetland-dominated streams: } \\
18 \%-43 \% \text { increase, reference } \\
\text { mean } 1980-1984 \\
\text { Increases mainly due to high } \\
\text { concentrations in last } 4 \text { years }\end{array}$ & 1980-2001 & $\begin{array}{l}\text { Ontario, } \\
\text { Canada }\end{array}$ \\
\hline [44] & streams & 7 & DOC & $\begin{array}{l}6 \text { wetland-dominated streams: } \\
\text { ss increase (HP3, HP5, HP6, PC1: } \\
p<0.01 \text {; HP4, HP6A: } p<0.05) \\
1 \text { upland-dominated system }(\mathrm{HP} 3 \mathrm{~A}) \text { : } \\
\text { no trend }\end{array}$ & $\begin{array}{l}\text { HP3: } 0.12 \\
\text { HP4: } 0.046 \\
\text { HP4: } 0.15 \\
\text { HP5: } 0.10 \\
\text { HP6: } 0.10 \\
\text { HP6A: } 0.094 \\
\text { PC1: } 0.12 \\
\end{array}$ & $\begin{array}{l}\text { Same data as in }[43,45] \\
\text { Different results when using } \\
\text { annual average or volume- } \\
\text { weighted concentrations. Here } \\
\text { volume-weighted shown }\end{array}$ & 1980-2001 & $\begin{array}{l}\text { Ontario, } \\
\text { Canada }\end{array}$ \\
\hline [45] & stream & 1 & DOC & ss increase $(p<0.01)$ & 0.12 & $\begin{array}{l}\text { Same data as }[43,44] \\
\text { Varying depending on season }\end{array}$ & 1980-2001 & $\begin{array}{l}\text { Ontario, } \\
\text { Canada }\end{array}$ \\
\hline [46] & rivers & 21 & TOC, A, COD & $\begin{array}{l}\text { TOC increase "smaller (than A, COD) } \\
\text { and negligible for some rivers", no stats } \\
\text { Several periodic reversals in the direction } \\
\text { of the trends }\end{array}$ & - & $\begin{array}{l}\text { Median annual TOC increase: } \\
0.27 \% \\
\text { Simultaneous behavior of TOC, } \\
\text { A and COD } \\
\text { Synchronicity among rivers }\end{array}$ & $\begin{array}{l}\text { TOC (21 rivers): } \\
\text { 1987-2004 } \\
\text { A, COD ( } 28 \text { rivers): } \\
\text { 1970-2004 }\end{array}$ & Sweden \\
\hline [47] & $\begin{array}{l}\text { lake } \\
\text { stream }\end{array}$ & $\begin{array}{l}1 \\
1\end{array}$ & DOC & $\begin{array}{l}\text { Lake: ss increase }(p<0.01) \\
\text { Stream: no trend }\end{array}$ & 0.19 & $\begin{array}{l}\text { Same system studied in [48] } \\
\text { where slope }=0.18 \\
\mathrm{mg} \mathrm{C} \mathrm{L}^{-1} \mathrm{y}^{-1} \text { for } 1987-2003\end{array}$ & 1990-2003 & Finland \\
\hline [49] & lakes & 12 & DOC & $\begin{array}{l}\text { Sudbury lakes (5): all ss increase } \\
\quad(p<0.05) \\
\text { Dorset lakes }(7): 3 \text { ss increase }(p<0.05)\end{array}$ & - & & $\begin{array}{l}\text { Sudbury: (1981, 1982, } \\
\text { 1987)-2003 } \\
\text { Dorset: } 1978 / 9-2003 \\
\end{array}$ & $\begin{array}{l}\text { Ontario, } \\
\text { Canada }\end{array}$ \\
\hline
\end{tabular}


Table 3. Cont.

\begin{tabular}{|c|c|c|c|c|c|c|c|c|}
\hline Ref. & $\begin{array}{l}\text { Type of } \\
\text { system } \\
\end{array}$ & No. & $\begin{array}{l}\text { Measured } \\
\text { parameter }^{b}\end{array}$ & Temporal trend? ${ }^{\mathrm{c}}$ & $\begin{array}{l}\text { Trend magnitude } \\
/ \mathrm{mgC} \mathrm{L}^{-1} \mathbf{y}^{-1}\end{array}$ & Comments & Period & Location \\
\hline$[50]$ & river & 1 & $\begin{array}{l}\text { TOC } \\
\text { estimated } \\
\text { from COD } \\
\text { values }\end{array}$ & No trend $(p>0.05)$ & - & $\begin{array}{l}\text { DON increased ss }(p<0.01) \\
(1982-2005) \\
\text { "Increases of DOC occurred } \\
\text { earlier (1970s-1980s) but } \\
\text { could not be quantified (low } \\
\text { sampling frequency)" }\end{array}$ & $1962-2005$ & Finland \\
\hline [51] & $\begin{array}{l}\text { lakes and } \\
\text { streams }\end{array}$ & $\begin{array}{l}522 \\
\text { (6 regions) }\end{array}$ & DOC & $\begin{array}{l}\text { 363: increase, no stats } \\
139: \text { decrease, no stats } \\
\text { " } 88 \% \text { of ss trends }(p<0.05) \text { were } \\
\text { positive" but nowhere is said how } \\
\text { many ss trends found }\end{array}$ & $\begin{array}{l}\text { Values represented in a } \\
\text { figure and in histograms } \\
\text { per region }\end{array}$ & $\begin{array}{l}\text { Upward ss slopes more frequent } \\
\text { below } 62^{\circ} \text { latitude in the UK } \\
\text { and in NE USA } \\
\text { Atlantic Canada little evidence } \\
\text { of increasing DOC }\end{array}$ & & $\begin{array}{l}\text { North } \\
\text { America and } \\
\text { northern } \\
\text { Europe }\end{array}$ \\
\hline [41] & streams & 2 & DOC & Increase, no stats & - & $\begin{array}{l}\text { Increasing amplitude of } \\
\text { seasonalvariations leading to a } \\
\text { long-term increase }\end{array}$ & 1983-2006 & $\begin{array}{l}\text { Scotland, } \\
\text { United } \\
\text { Kingdom }\end{array}$ \\
\hline$[52]^{\mathrm{f}}$ & streams & 3 & TOC & $\begin{array}{l}\text { All ss increase: Langtjern }(p<0.008), \\
\text { Birkenes }(p<0.002) \text {, Storgama } \\
(p<0.001)\end{array}$ & $\begin{array}{l}\text { Langtjern: } 0.13 \\
\text { Birkenes: } 0.06 \\
\text { Storgama: } 0.09\end{array}$ & $\begin{array}{l}\text { All period increases (trend } \\
\text { divised by mean TOC): } \\
\text { Langtjern: } 14 \% \text {, Birkenes: } \\
22 \% \text {, Storgama: } 36 \% \\
\end{array}$ & 1985-2003 & Norway \\
\hline [53] & $\begin{array}{l}\text { lakes and } \\
\text { rivers }\end{array}$ & 117 & $\begin{array}{l}\text { DOC; in some } \\
\text { cases, DOC } \\
\text { deduced } \\
\text { from color } \\
\text { (2 sites) or } \\
\text { COD values } \\
\text { (42 sites) }\end{array}$ & $\begin{array}{l}1977-2002 \text { ( } 54 \text { sites): } 12 \text { increase, } \\
23 \text { decrease, } 19 \text { no trend; no stats } \\
1977-1986 \text { ( } 51 \text { sites): } 7 \text { increase, } \\
16 \text { decrease, } 27 \text { no trend; no stats } \\
1993-2002 \text { ( } 94 \text { sites): } 5 \text { increase, } \\
56 \text { decrease, } 33 \text { no trend; no stats }\end{array}$ & $\begin{array}{l}1977-2002:-0.04-0.02^{\mathrm{j}} \\
1993-2002:-0.19-0.08^{\mathrm{j}}\end{array}$ & $\begin{array}{l}198 \text { sites from [47] also } \\
\text { considered }\end{array}$ & 1977-2002 & $\begin{array}{l}\text { United } \\
\text { Kingdom }\end{array}$ \\
\hline
\end{tabular}


Table 3. Cont.

\begin{tabular}{|c|c|c|c|c|c|c|c|c|}
\hline Ref. & $\begin{array}{l}\text { Type of } \\
\text { system }\end{array}$ & No. & $\begin{array}{l}\text { Measured } \\
\text { parameter }^{b}\end{array}$ & Temporal trend? ${ }^{\mathrm{c}}$ & $\begin{array}{l}\text { Trend magnitude } \\
/ \mathrm{mgC} \mathrm{L}^{-1} \mathbf{y}^{-1}\end{array}$ & Comments & Period & Location \\
\hline [55] & $\begin{array}{l}\text { lakes } \\
\text { streams }\end{array}$ & $\begin{array}{l}12 \\
5\end{array}$ & DOC & $\begin{array}{l}75 \% \text { lakes: ss increase }(p<0.05) \\
80 \% \text { streams: ss increase }(p<0.05)\end{array}$ & $\begin{array}{l}\text { Lake mean: } 0.091^{\mathrm{g}} \\
\text { Stream mean: } 0.056^{\mathrm{g}}\end{array}$ & $\begin{array}{l}\text { Trends nss counted as a trend of } \\
0 \text { when calculating mean trend } \\
\text { values }\end{array}$ & $1992-2001$ & USA \\
\hline [15] & $\begin{array}{l}\text { lakes } \\
\text { streams }\end{array}$ & $\begin{array}{l}11 \\
11\end{array}$ & DOC & $\begin{array}{l}\text { In all: ss increase (Mann-Whitney), } \\
\text { most } p<0.001\end{array}$ & - & $\begin{array}{l}\text { Average increase last first } 5 \\
\text { years compared to } 5 \text { last years: } \\
\text { lakes: } 63 \% \text {, streams: } 71 \% \\
\text { Same results as [16] expressed } \\
\text { otherwise }\end{array}$ & $1988-2003$ & $\begin{array}{l}\text { United } \\
\text { Kingdom }\end{array}$ \\
\hline [48] & $\begin{array}{l}\text { lakes } \\
\text { streams }\end{array}$ & $\begin{array}{l}13 \\
2\end{array}$ & $\begin{array}{l}\text { TOC, }<1990 \\
\text { deduced } \\
\text { from COD }\end{array}$ & $\begin{array}{l}6 \text { lakes: ss increase }(p<0.001) \\
3 \text { lakes: ss increase }(p<0.05) \\
1 \text { lake: ss increase }(p<0.1) \\
3 \text { lakes: no trends } \\
1 \text { stream: ss increase }(p<0.0001)\end{array}$ & $\begin{array}{l}\text { Lakes: } 0.10,0.08,0.14, \\
0.22,0.18,0.03,0.11, \\
0.12,0.04,0.12 \\
\text { Stream: } 0.35\end{array}$ & $\begin{array}{l}\text { Lakes with ss increase include } \\
\text { both clear water and humic } \\
\text { lakes } \\
\text { Poor correlation ( } \mathrm{r}=0.30 \text {, } \\
p=0.33 \text { ) between annual TOC } \\
\text { increase and initial [TOC] }\end{array}$ & $1987-2003$ & Finland \\
\hline$[31]$ & stream & $\begin{array}{l}1 \\
(2 \text { sampling } \\
\text { sites })\end{array}$ & DOC & $\begin{array}{l}\text { Trout Beck: "almost step change from } \\
1995 \text { to } 1997 \text { with little subsequent } \\
\text { decline in values", no stats } \\
\text { Cottage Hill Sike: similar but "with } \\
\text { more evidence of a decline after } \\
\text { 1997", no stats }\end{array}$ & - & $\begin{array}{l}\text { Trout Beck, same data as in [30] } \\
\text { but different conclusions }\end{array}$ & 1994-2001 & $\begin{array}{l}\text { United } \\
\text { Kingdom }\end{array}$ \\
\hline [56] & lakes & 7 & DOC & Oscilations (3-year running means) & - & $\begin{array}{l}\text { Oscillations in annual water } \\
\text { discharge and total DOC load } \\
\text { were similar in the } 7 \text { lakes; } \\
\text { annual [DOC] variations were } \\
\text { similar but less accentuated }\end{array}$ & $1978-1998$ & $\begin{array}{l}\text { Ontario, } \\
\text { Canada }\end{array}$ \\
\hline
\end{tabular}


Table 3. Cont

\begin{tabular}{|c|c|c|c|c|c|c|c|c|}
\hline Ref. & $\begin{array}{l}\text { Type of } \\
\text { system }\end{array}$ & No. & $\begin{array}{l}\text { Measured } \\
\text { parameter }^{b}\end{array}$ & Temporal trend? ${ }^{\mathrm{c}}$ & $\begin{array}{l}\text { Trend magnitude } \\
\text { / } \mathrm{mgC} \mathrm{L}^{-1} \mathbf{y}^{-1}\end{array}$ & Comments & Period & Location \\
\hline [16] & $\begin{array}{l}\text { lakes } \\
\text { streams }\end{array}$ & $\begin{array}{l}11 \\
11\end{array}$ & DOC & All sites: ss increases, no stats & Range: $0.06-0.51$ & $\begin{array}{l}\text { In all sites, annual }[\mathrm{DOC}] \text { correlated } \\
\text { to mean }[\mathrm{DOC}] \text { for first } 5 \text { years } \\
\left(\mathrm{r}^{2}=0.71\right) \\
\text { All period, } 91 \% \text { increase relative to } \\
1988-1993 \text { mean }\end{array}$ & $1988-2003$ & $\begin{array}{l}\text { United } \\
\text { Kingdom }\end{array}$ \\
\hline [57] & river & $\begin{array}{l}1 \\
\text { (6 stations) }\end{array}$ & DOC & All sites: ss increase $(p<0.001)$ & - & $\begin{array}{l}\text { "DOC concentrations have doubled } \\
\text { from } 1988 \text { to } 2003 " \\
\text { "Net change between } 3 \text { and } \\
4 \mathrm{mg} \mathrm{C} \mathrm{L} \text { "- } \\
\text { Decrease in downstream decline }\end{array}$ & $1988-2003$ & USA \\
\hline [23] & streams & $\begin{array}{l}3 \\
\text { (6 sampling } \\
\text { sites) }\end{array}$ & DOC & $\begin{array}{l}\text { "significant upwards trend" } \\
(p: 0.000-0.023)\end{array}$ & $\begin{array}{c}0.056,0.058,0.055 \\
0.047,0.051,0.045 \\
0.146,0.055,0.019\end{array}$ & $\begin{array}{l}\text { Data filtered for season, air T, flow: } \\
\text { residual trend for } 1983-1993 \text { and } \\
\text { levelling off from } 1983 \text { onwards } \\
\text { (streams draining forest) }\end{array}$ & $\begin{array}{l}(1983,1984,1988 \\
1990,1991)-2002\end{array}$ & $\begin{array}{l}\text { United } \\
\text { Kingdom }\end{array}$ \\
\hline$[18]^{\mathrm{h}}$ & sites & $\begin{array}{l}189 \\
(12 \text { regions })\end{array}$ & DOC & $\begin{array}{l}6 \text { regions }(n=121) \text { : ss increase } \\
(p<0.05) \\
4 \text { regions }(n=59) \text { : no trends } \\
1 \text { region (Virginia Blue Ridge) }(n=3) \text { : } \\
\text { ss decrease }(p<0.05) \\
1 \text { region (Alps) }(n=6) \text { : insufficient } \\
\text { data }\end{array}$ & $\begin{array}{l}0.05,0.08,0.13,0.06 \\
0.06,0.06,-0.04\end{array}$ & $\begin{array}{l}\text { Europe: ss increase in Nordic } \\
\text { countries and UK, nss in central } \\
\text { Europe } \\
\text { N. America: ss increase in } \\
\text { Vermont/Quebec, Adirondacks, } \\
\text { Upper Midwest; nss Maine/Atlantic } \\
\text { Canada, Appalachian }\end{array}$ & 1990-2001 & $\begin{array}{l}\text { Europe and } \\
\text { North } \\
\text { America }\end{array}$ \\
\hline [58] & rivers & 16 & TOC & $\begin{array}{l}\text { 10: ss decrease }(p<0.05) \text { at least once } \\
\text { during March, May, Aug, Oct }\end{array}$ & - & $\begin{array}{l}\text { Some ss decrease observed in: } 8 \\
\text { rivers only for } 1 \text { period, } 1 \text { for } 2 \text { and } \\
1 \text { for } 3 \text {; in total: in } 13 \text { of the } 64 \\
\text { periods considered }\end{array}$ & $1975-2000$ & Finland \\
\hline [59] & lake & 1 & $\begin{array}{l}\text { DOC, color, } \\
\text { COD }\end{array}$ & $\begin{array}{l}\text { DOC, COD increased since } 1990 \text { (no } \\
\text { stats) } \\
\text { Color increased 1976-2002 (no stats) } \\
\text { but not continuously }\end{array}$ & - & $\begin{array}{l}\text { After 2000, color declined (more than } \\
40 \% \text { ), COD and DOC } 11 \%-13 \%\end{array}$ & $\begin{array}{l}\text { From } 1976 \text { (color), } \\
1982 \text { (COD), } 1989 \\
\text { (DOC) to } 2002\end{array}$ & Norway \\
\hline
\end{tabular}


Table 3. Cont.

\begin{tabular}{|c|c|c|c|c|c|c|c|c|}
\hline Ref. & $\begin{array}{l}\text { Type of } \\
\text { system }\end{array}$ & No. & $\begin{array}{l}\text { Measured } \\
\text { parameter }^{b}\end{array}$ & Temporal trend? ${ }^{c}$ & $\begin{array}{l}\text { Trend magnitude } \\
/ \mathrm{mgC} \mathrm{L}^{-1} \mathbf{y}^{-1}\end{array}$ & Comments & Period & Location \\
\hline [47] & $\begin{array}{l}\text { lakes } \\
\text { supply } \\
\text { reservoirs } \\
\text { streams } \\
\text { and rivers }\end{array}$ & $\begin{array}{l}29 \\
8 \\
161\end{array}$ & DOC & $\begin{array}{l}\text { 153: ss increase }(p<0.05) \\
45: \text { no trends }\end{array}$ & Mean all sites: 0.17 & & $\begin{array}{l}\text { Variable-2000; some } \\
\text { from 1962, most } 10 \\
\text { years long }\end{array}$ & $\begin{array}{l}\text { United } \\
\text { Kingdom }\end{array}$ \\
\hline [30] & stream & 1 & DOC & Increase, no stats & $\begin{array}{l}0.62 \text { (annual median } \\
\text { increase) }\end{array}$ & & $1992-2000$ & $\begin{array}{l}\text { United } \\
\text { Kingdom }\end{array}$ \\
\hline [60] & river & 2 & Color & Increase, no stats & - & $\begin{array}{l}\text { Tees median: } \\
1.83 \text { Hazen units } \mathrm{y}^{-1} \\
\left(\approx 0.11 \mathrm{mg} \mathrm{C} \mathrm{L}{ }^{-1} \mathrm{y}^{-1}\right) \\
\text { Coquet median: } \\
0.52 \text { Hazen units } \mathrm{y}^{-1} \\
\left(\approx 0.026 \mathrm{mg} \mathrm{C} \mathrm{L}^{-1} \mathrm{y}^{-1}\right) \\
\text { Although this study uses exactly } \\
\text { the same data set as [61], } \\
\text { results for Coquet River differ; } \\
\text { no reason given }\end{array}$ & $\begin{array}{l}\text { Tees: } 1970-2000 \\
\text { Coquet: } 1962-2001\end{array}$ & $\begin{array}{l}\text { United } \\
\text { Kingdom }\end{array}$ \\
\hline$[62]$ & lakes & 52 & DOC & $\begin{array}{l}1982-2000(16 \text { not limed }): 7 \text { ss increase, } \\
1 \text { ss decrease }(p<0.1) \\
1992-2000(48 \text { not limed): } 7 \text { ss increase, } \\
41 \text { no trend }(p<0.1)\end{array}$ & $\begin{array}{l}\text { Mean rate of DOC } \\
\text { increase 1982-2000: } \\
0.079^{\mathrm{g}}\end{array}$ & $\begin{array}{l}\text { 1982-2000: "the rate of DOC } \\
\text { increase more rapid at higher } \\
\text { lake [DOC]" }\end{array}$ & $\begin{array}{l}\text { 1982-2000 (17 lakes) } \\
1992-2000 \text { (52 lakes) }\end{array}$ & USA \\
\hline [64] & lakes & 163 & TOC & $\begin{array}{l}0 \%-10 \% \text { of lakes in different regions: } \\
\text { ss increase }(p<0.05) \\
\text { Most: no trend }\end{array}$ & Values in a figure & & 1990-1999 & Finland \\
\hline
\end{tabular}


Table 3. Cont

\begin{tabular}{|c|c|c|c|c|c|c|c|c|}
\hline Ref. & $\begin{array}{l}\text { Type of } \\
\text { system }\end{array}$ & No. & $\begin{array}{l}\text { Measured } \\
\text { parameter }^{\mathbf{b}}\end{array}$ & Temporal trend? ${ }^{\mathrm{c}}$ & $\begin{array}{l}\text { Trend magnitude } \\
\text { / }^{\mathrm{d}} \mathrm{gC} \mathrm{L}^{-1} \mathbf{y}^{-1}\end{array}$ & Comments & Period & Location \\
\hline [65] & stream & 1 & COD & $\begin{array}{l}\text { 1969-2000: ss increase }(p<0.05) \\
\text { 1969-1984: ss decrease }(p<0.01) \\
\text { 1983-2000: ss increase }(p<0.01)\end{array}$ & - & COD slopes & 1969-2000 & $\begin{array}{l}\text { Czech } \\
\text { Republic }\end{array}$ \\
\hline$[35]$ & lakes & 9 & DOC & $\begin{array}{l}\text { "Common pattern: concentrations were } \\
\text { higher between } 1978 \text { and } 1982 \text { and from } \\
1990 \text { to } 1997 "\end{array}$ & - & & 1978-1998 & $\begin{array}{l}\text { Ontario, } \\
\text { Canada }\end{array}$ \\
\hline [66] & lakes & 705 & DOC & $\begin{array}{l}\text { Quebec }(n=43): 14 \% \text { increase, } 10 \% \\
\text { decrease, } 76 \% \text { no trend }(p<0.10) \\
\text { Ontario }(n=662): 4 \% \text { increase, } 5 \% \\
\text { decrease, } 91 \% \text { no trend }(p<0.05) \\
\text { Subset Ontario no CWS }(n=54): 22 \% \\
\text { increase, } 4 \% \text { decrease, no } 74 \% \text { trend } \\
(p<0.05)\end{array}$ & - & & $\begin{array}{l}\text { Quebec: } 1990-1997 \\
\text { Ontario: 1990-1999 }\end{array}$ & Canada \\
\hline [67] & lakes & 8 & DOC & $\begin{array}{l}1(\text { Johnnie }) \text { : ss increase }(p<0.05) \\
7: \text { no trend }\end{array}$ & - & & 1988-2001 & $\begin{array}{l}\text { Ontario, } \\
\text { Canada }\end{array}$ \\
\hline [41] & $\begin{array}{l}\text { lake } \\
\text { streams }\end{array}$ & $\begin{array}{l}1 \\
8 \text { (one with } \\
7 \text { sites) } \\
\end{array}$ & DOC & All sites: ss increase, no stats & - & & $\begin{array}{l}\text { Loch Ard: } 1977-2000 \\
\text { Loch Grannoch: } \\
\text { 1978-present }\end{array}$ & $\begin{array}{l}\text { Scotland, } \\
\text { United } \\
\text { Kingdom }\end{array}$ \\
\hline [61] & rivers & 3 & Color & $\begin{array}{l}\text { Tees: ss increase, no stats } \\
\text { Wear: no trend, no stats } \\
\text { Coquet: ss increase, no stats }\end{array}$ & - & $\begin{array}{l}\text { Tees increase: } 51 \text { Hazen units } \\
\text { (29 years), } 1.75 \text { Hazen units } \\
\mathrm{y}^{-1}\left(\approx 0.1 \mathrm{mg} \mathrm{C} \mathrm{L}^{-1} \mathrm{y}^{-1}\right) \\
\text { Coquet increase: } 29 \text { Hazen } \\
\text { units ( } 39 \text { years), } 61 \% \\
\text { "Annual averages for the } 3 \\
\text { sites show a clear common } \\
\text { phase" }\end{array}$ & $\begin{array}{l}\text { Tees: } 1970-2000 \\
\text { Wear: } 1969-1998 \\
\text { Coquet: } 1962-2000\end{array}$ & $\begin{array}{l}\text { United } \\
\text { Kingdom }\end{array}$ \\
\hline
\end{tabular}


Table 3. Cont

\begin{tabular}{|c|c|c|c|c|c|c|c|c|}
\hline Ref. & $\begin{array}{l}\text { Type of } \\
\text { system }\end{array}$ & No. & $\begin{array}{l}\text { Measured } \\
\text { parameter }^{\text {b }}\end{array}$ & Temporal trend? ${ }^{c}$ & $\begin{array}{l}\text { Trend magnitude } \\
\text { / } \mathbf{m g C ~ L}^{-1} \mathbf{y}^{-1}\end{array}$ & Comments & Period & Location \\
\hline [14] & $\begin{array}{l}\text { lakes } \\
\text { streams }\end{array}$ & $\begin{array}{l}11 \\
11\end{array}$ & DOC & 20: ss increase $(p<0.05)$ & - & $\begin{array}{l}\text { Annual average increases }(5.4 \%) \\
\text { proportional to mean }[\mathrm{DOC}] \\
\left(\mathrm{R}^{2}=0.81, p<0.001\right)\end{array}$ & $1989-2000$ & $\begin{array}{l}\text { United } \\
\text { Kingdom }\end{array}$ \\
\hline [68] & lakes & 4 & $\begin{array}{l}\text { Secchi disk } \\
\text { depth }\end{array}$ & $\begin{array}{l}\text { Nellie, OSA: ss Secchi depth increase } \\
\quad(p<0.05) \\
\text { George: decrease, no stats } \\
\text { Bell Lake: nss change }(p>0.05)\end{array}$ & - & George: $-0.1 \mathrm{~m} \mathrm{yr}^{-1}$ & 1969-1999 & $\begin{array}{l}\text { Ontario, } \\
\text { Canada }\end{array}$ \\
\hline [69] & $\begin{array}{l}\text { lakes } \\
\text { streams }\end{array}$ & $\begin{array}{l}21 \\
16\end{array}$ & DOC & 36: ss increase, no stats & $\begin{array}{l}\text { Median annual trend } \\
\text { values in a figure }\end{array}$ & $\begin{array}{l}\text { Site with the lowest DOC, the } \\
\text { only one with nss increase } \\
\text { Greatest annual DOC changes in } \\
\text { sites with most highly colored } \\
\text { waters }\end{array}$ & $(1972-1988)-2000$ & $\begin{array}{l}\text { Scotland, } \\
\text { United } \\
\text { Kingdom }\end{array}$ \\
\hline [70] & $\begin{array}{l}\text { "ICP } \\
\text { Waters" } \\
\text { sites }\end{array}$ & 98 & DOC & $\begin{array}{l}\text { Northern Nordic Countries }(n=6) \text { : no trend } \\
\text { Nordic Countries/UK }(n=24) \text { : ss increase } \\
\quad(p<0.001) \\
\text { Central Europe }(n=34) \text { : no trend } \\
\text { Eastern North America }(n=22) \text { : ss increase } \\
\quad(p<0.01) \\
\text { Midwestern North America }(n=9) \text { : ss } \\
\text { increase }(p<0.001)\end{array}$ & $\begin{array}{l}- \\
4.8 \times 10^{-4 \mathrm{i}} \\
- \\
3.6 \times 10^{-4 \mathrm{i}} \\
1.2 \times 10^{-4 \mathrm{i}}\end{array}$ & & 1989-1996 & $\begin{array}{l}\text { Europe and } \\
\text { North } \\
\text { America }\end{array}$ \\
\hline [71] & lakes & 344 & TOC & $\begin{array}{l}42(12 \%) \text { : ss increase }(p<0.05) \\
4: \text { ss decrease }(p<0.05) \\
87 \% \text { : no trend }\end{array}$ & Values in a figure & $\begin{array}{l}\text { Lakes with increases located in } \\
\text { SE Norway, S Sweden and in a } \\
\text { few cases S Finland }\end{array}$ & 1990-1999 & Scandinavia \\
\hline [72] & rivers & 9 & Color, COD & $\begin{array}{l}\text { Color: } 4 \text { : ss decrease }(p<0.05) ; 2 \text { : ss } \\
\text { increase }(p<0.05) ; 3 \text { : no trend } \\
\text { COD: } 7 \text { : ss decrease }(p<0.05) ; 1 \text { : ss } \\
\text { decrease }(p<0.1) ; 1 \text { : no trend }\end{array}$ & - & $\begin{array}{l}\text { Color (in Pt scale) and COD } \\
\text { slopes }\end{array}$ & $1977-1995$ & Latvia \\
\hline
\end{tabular}


Table 3. Cont

\begin{tabular}{|c|c|c|c|c|c|c|c|c|}
\hline Ref. & $\begin{array}{l}\text { Type of } \\
\text { system }\end{array}$ & No. & $\begin{array}{l}\text { Measured } \\
\text { parameter }^{\text {b }}\end{array}$ & Temporal trend? ${ }^{c}$ & $\begin{array}{l}\text { Trend magnitude } \\
\text { / } \mathbf{m g C ~ L}^{-1} \mathbf{y}^{-1}\end{array}$ & Comments & Period & Location \\
\hline [73] & lakes & 155 & DOC & $\begin{array}{l}3 \% \text { : ss increase, no stats } \\
5 \% \text { : ss decrease, no stats } \\
92 \% \text { : no trend }\end{array}$ & $\begin{array}{l}\text { Median: }-0.11 \\
\text { Range: }-0.75 \text { to } 0.42^{j}\end{array}$ & & $1983-1995$ & $\begin{array}{l}\text { Ontario, } \\
\text { Canada }\end{array}$ \\
\hline [74] & lakes & 37 & DOC & $\begin{array}{l}17: \text { ss increase }(p=0.1) \\
1: \text { ss decrease }(p=0.1) \\
\text { 19: no trend }\end{array}$ & - & $\begin{array}{l}\text { Net changes }(1985-1993) \text { by } \\
\text { region: } \\
\text { R1 }(n=6):+1.7 \mu \mathrm{M} \mathrm{C} \\
\text { R2 }(n=8):+66.6 \mu \mathrm{M} \mathrm{C} \\
\text { R3 }(n=8):+81.6 \mu \mathrm{M} \mathrm{C} \\
\text { R4 }(n=5):- \\
\text { R5 }(n=4):- \\
\text { R6 }(n=6):+55.8 \mu \mathrm{M} \mathrm{C}\end{array}$ & $(1983,1986,1989)-1993$ & $\begin{array}{l}\text { Quebec, } \\
\text { Canada }\end{array}$ \\
\hline [75] & $\begin{array}{l}\text { lakes } \\
\text { streams }\end{array}$ & $\begin{array}{l}3 \\
4\end{array}$ & DOC & $\begin{array}{l}\text { Lakes: decrease, no stats } \\
\text { Streams: increase, no stats }\end{array}$ & & $\begin{array}{l}\text { Lakes: "DOC concentrations } \\
\text { declined by } 15 \%-25 \% " \\
\text { Streams: "average } \\
\text { concentrations increased by } \\
30 \%-80 \% "\end{array}$ & $\begin{array}{l}\text { Early } 70 \text { 's }-1990, \\
\text { depending on system }\end{array}$ & $\begin{array}{l}\text { Ontario, } \\
\text { Canada }\end{array}$ \\
\hline [63] & lakes & 17 & DOC & $\begin{array}{l}\text { 4: ss decrease }(p<0.1) \\
13: \text { no trend }\end{array}$ & $\begin{array}{l}-0.072^{\mathrm{i}} \text { (Constable), } \\
-0.108^{\mathrm{i}} \text { (Windfall), } \\
-0.144^{\mathrm{i}} \text { (Heart), } \\
-0.156^{\mathrm{i}} \text { (Squash) }\end{array}$ & & 1982-1992 & USA \\
\hline [76] & $\begin{array}{l}\text { lake } \\
\text { rivers }\end{array}$ & $\begin{array}{l}1 \\
7\end{array}$ & $\begin{array}{l}\text { Lake: color } \\
\text { Rivers: A(420 } \\
\text { nm) }\end{array}$ & $\begin{array}{l}\text { Linear decrease from the end of the } \\
1960 \text { 's to the beginning of the } 1970 \text { 's, } \\
\text { followed by an almost linear increase } \\
\text { up to } 1988\end{array}$ & & & $\begin{array}{l}\text { Lake: } 1960-1988 \\
\text { River Alsterälven: } 1968- \\
1987\end{array}$ & Sweden \\
\hline
\end{tabular}


Table 3. Cont

\begin{tabular}{|c|c|c|c|c|c|c|c|c|}
\hline Ref. & $\begin{array}{l}\text { Type of } \\
\text { system }\end{array}$ & No. & $\begin{array}{l}\text { Measured } \\
\text { parameter }^{\text {b }}\end{array}$ & Temporal trend? $^{c}$ & $\begin{array}{l}\text { Trend magnitude } \\
\text { / } \mathrm{mgC} \mathrm{L}^{-1} \mathbf{y}^{-1}\end{array}$ & Comments & Period & Location \\
\hline [77] & $\begin{array}{l}\text { lakes } \\
\text { rivers }\end{array}$ & $\begin{array}{l}283 \\
18\end{array}$ & $\begin{array}{l}\text { Lakes: color } \\
\text { Rivers: A(420 } \\
\text { nm) }\end{array}$ & $\begin{array}{l}\text { Lakes: increase, no stats } \\
\text { Rivers: ss increase in } 17 \text { (95\% level) }\end{array}$ & & $\begin{array}{l}\text { Lakes: average increase: } \\
20 \mathrm{mg} \mathrm{Pt} \mathrm{L}^{-1} \text {, marked increase } \\
(\approx 100 \%) \text { in large areas of } \mathrm{N} \\
\text { and S Sweden } \\
\text { Rivers: relative increase: } \\
12 \%-150 \% \text {; largest increases } \\
\text { in the smallest drainage areas; } \\
\text { increase appears to be a part } \\
\text { of long-term variations } \\
\text { (oscillations); no distinct } \\
\text { geographical distribution } \\
\text { pattern }\end{array}$ & $\begin{array}{l}\text { Lakes: 1972-1987 } \\
\text { Rivers: 1972-1986 (some } \\
\text { 1965) }\end{array}$ & Sweden \\
\hline [78] & lakes & 4 & Color & Increase, no stats & & $\begin{array}{l}\text { Oxsjön: } 10 \text { to } 20 \mathrm{mg} \mathrm{Pt} \mathrm{L}-1 \\
\text { Hammardammen: }<30 \text { to }>40 \\
\text { Innaren: } 0-10 \text { to } 11-20 \\
\text { Värmen: } 20 \text { to } 50\end{array}$ & $\begin{array}{l}\text { Oxsjön: 1967-1982 } \\
\text { Hammardammen: 1972- } \\
1988 \\
\text { Innaren: 1970's-1980's } \\
\text { Värmen: 1976-1986 }\end{array}$ & Sweden \\
\hline [79] & stream & 1 & $\mathrm{~A}(400 \mathrm{~nm})$ & No long-term trend, no stats & & $\begin{array}{l}\text { Short term increases in } 1980 \text {, } \\
\text { 1985, } 1987 \\
\text { Tendency towards more } \\
\text { extreme values }\end{array}$ & 1979-1987 & $\begin{array}{l}\text { United } \\
\text { Kingdom }\end{array}$ \\
\hline
\end{tabular}

Notes: ${ }^{\mathrm{a}}$ Complementary information in Tables 1 and $2{ }^{\mathrm{b}}$ In general, the DOC/TOC term used by the authors has been kept except when the term DOC had been used in studies where it is clear that samples were unfiltered; ${ }^{c}$ ss $=$ statistically significant, nss $=$ not statistically significant. Non statistically increases and decreases are considered "no trends"; ${ }^{\mathrm{d}}$ Values only given when trend statistically significant, except when mixed in the original publication (in this case, a cautionary note is added). When value in italics, original value in other units; ${ }^{\mathrm{e}}$ [88] includes data from two streams (Loch Ard Burn 2 and Allt a'Mharcaidh) but refers to this study for DOC long term trend values; ${ }^{\mathrm{f}}$ Data from one of the catchments (Langtjern) further treated in [89] by

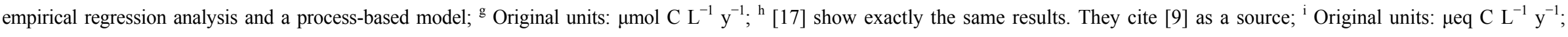
${ }^{\mathrm{j}}$ Probably slopes for non statistically significant trends included. 


\subsubsection{Limited Geographical Location}

Not many long series of reliable OC data exist. An exception is data collected in association with the follow up of a given problem. This is the case of extensive surveys in northern US and European countries related to acid deposition effects. As a consequence, and as Table 1 shows, many of the published studies on OC trends have been obtained in systems affected by acid rain. This means that (i) these systems are located geographically in a limited zone of the planet and that, as a consequence, they are often climatically similar; (ii) the type of organic matter present in the water bodies is similar (i.e., the bodies are mostly rich in humic-type compounds with limited concentrations of other types of organic matter linked to productivity [90,91]). Published studies are distributed as follows: United Kingdom (19, most in Scotland and northern England), Canada (15, mostly in Ontario and Quebec), USA (5, all in the East Coast), Scandinavian countries (13), Czech Republic (3), Estonia (1), Germany (1), Latvia (1), Netherlands (1), Switzerland (1). Three studies [18,51,71] cover a large number of systems over Europa and North America but located in the same climatic zones as the smaller size studies mentioned. Apart from the limited geographical covering of the existing studies, the fact that these OC concentrations have been measured in systems recovering from a strong chemical disturbance such as acid rain means that the main trends observed will be directly related to the chemical changes in water composition associated with the main system modification (either acidification or recovery), eliminating, or at least greatly reducing, any possibility of detecting trends linked to global climatic changes.

\subsection{Analytical Aspects}

Organic carbon concentration is a particularly "difficult" parameter because it is highly dependent on the measurement method used. For this reason, it is essential to know, and to understand, this aspect of published studies in order to be able to evaluate the validity and meaning of their conclusions. Methodological information concerning analytical aspects is gathered in Table 2. It includes filtration and information about the analytical method used to determine OC or any other surrogate parameter considered.

\subsubsection{On TOC, POC and DOC}

The simplest classification of the total organic carbon (TOC) pool includes TOC, to be split into particulate organic carbon (POC) and dissolved organic carbon (DOC), both fractions being obtained by filtration through a filter with a nominal pore size of 0.45 (usual in freshwaters) or $0.22 \mu \mathrm{m}$ (more common in oceanography). Astonishingly, in a majority of the studies ( $42 \%-67 \%$ of the total) filtration is not even mentioned. This can never be justified. When filtration is mentioned, the pore size $(0.45 \mu \mathrm{m})$ is given in only six articles and the type of filters without pore size in four further ones. In nine articles it is explicitly said that water was unfiltered and in six of them unfiltered OC is measured and called DOC on the basis that POC only accounts for 5\%-10\% of TOC in the waters studied. This might be reasonable in humic-type waters, such as the ones existing in higher latitude zones where most of the studies have been performed, but it cannot be assumed in water systems in other climatic 
zones. The fact that DOC and POC may evolve differently over time $[20,28]$ also needs to be taken into account when considering temporal trends, even in low-POC containing waters.

\subsubsection{Organic Carbon Concentration Measurements}

OC measurements have never been straightforward. Progress in the measurement of OC concentrations has been led mainly by oceanographers who developed and introduced the high-temperature catalytic oxidation (HTC) method for low OC concentration measurements in the late 1980s [92]. After some problems due to inappropriate blank estimates in the first publications that forced their authors to withdraw their data [93], the HTC technique imposed itself as the technique of choice in the field, for both seawater and freshwater. However, when considering long-term series of OC data, it is unavoidable that a significant part of the data has been obtained by other techniques, mainly through the wet oxidation method (WCO), based on the chemical oxidation of organic compounds by persulfate, combined or not with photo-oxidation using ultraviolet light. What are the implications? First, as pointed out by Dafner and Wangersky [94], when using older DOC data, one should be aware that values are likely to be in the right neighborhood but with a greater variability than we would now accept. A typical range of error of the DOC measurements by a recent HTC apparatus is $1 \%-2 \%$ as a relative value, which corresponds to approximately $1 / 10$ the error of the WCO method [95]. However, what it is less clear is whether both methods, irrespective of their inherent variability, produce (and previously produced!) similar results. To our knowledge, definitive large scale intercomparison tests have never been performed, although different authors conducted comparisons with variable results, ranging from no significant difference [96], low underestimation $(3 \%-6 \%$, [97]) or high underestimation $(20 \%-24 \%$, [83]) by the WCO method. Matters become even more complicated when we consider that, even within a single method, there are response variations over time. For instance, a progressive increase in DOC concentrations has been observed along with the use of progressively stronger oxidants in the WCO method [98].

Another method, used only by Dillon and co-workers [35,43-45,49,56] in Canadian lakes employs colorimetry with phenolphthalein as a detection method after UV oxidation of $\mathrm{OC}$ to $\mathrm{CO}_{2}$ in acidic persulfate media. How this method compares with the usual infrared detection of $\mathrm{CO}_{2}$ in $\mathrm{WCO}$ and HTC methods is unknown.

In the case of the time series considered here, two further questions arise. One, with no clear answer, is whether it is valid to compare results from different studies when obtained using different analytical methods for OC measurement. The second is how the fact of using data obtained with different methods within the same study series is dealt with and what effects this has on the trends reported. Sometimes authors mention that the methods used have been intercalibrated for a period of time (e.g., [39,57,75]) but not all authors report intercalibration (e.g., [36,38,48,59] do not). It is worth mentioning the case of [39] who studied OC trends with and without correcting old wet persulfate data and obtained quite different results (Table 3).

A much more controversial issue is the use of surrogate parameters such as color (expressed in so-called Hazen or Pt units, equivalent to the platinum concentration in a standard solution of platinum/cobalt chloride salts of the same absorbance/color), absorbance $(250,400$, or $420 \mathrm{~nm})$ or COD (chemical oxygen demand). This is highly controversial for two reasons. The first one is 
methodological, concerning how the link is made with "real" OC concentrations. The second reason concerns the validity of the hypothesis underlying all these studies: that $\mathrm{OC}$ and the surrogate parameter evolve in the same way with time.

Concerning methodological issues, some studies follow tendencies directly in the surrogate parameter as such $[26,60,61,65,72,77]$ but a common practice is to establish an empirical correlation between the parameter values and OC concentrations using data simultaneously measured over a short period of time $[29,38,48,50,53,61]$ and discuss tendencies, causes, etc. in terms of OC concentrations. Often these correlations are just used to explain the period where OC concentrations have been directly measured (Table 3). When given, correlations used are provided in Table 2. In one case [53], the authors use correlations established in other studies on the assumption that they will also apply to their systems. This approach is difficult to justify. Even extrapolating synchronous data between close systems seems questionable. For instance, Pärn and Mander showed that TOC and COD for rivers in Estonia, a small country, showed different Spearman's correlation coefficients depending on the area considered (much higher in the North than in the South) [26].

Definitively more open to discussion is the underlying hypothesis that both OC and the measured parameter will change over the years in the same way. Consider, for instance, that Apsite and Klavins [72] showed that statistically significant increasing trends obtained when considering color became statistically significant decreasing trends when considering COD. The surrogate parameters used such as color, absorbance, etc. only respond to a fraction of the organic matter present in the system, usually the fraction more refractory to degradation fraction, often known as humic substances [99,100]. Surprisingly, this fact is rarely acknowledged in the studies considered, with rare exceptions such as [29]. Probably, in many of the systems considered in the studies evaluated here, these types of substances account for the bulk of the OC present (see Section 3.1.2) and, thus, the hypothesis that both change in the same direction, and in the same magnitude, might be applicable. Nevertheless, this remains unproved and the few studies where, directly or indirectly, the question of the quality of the organic matter present has been addressed, do not seem to support this hypothesis. For instance, Dawson et al. [88] showed a significant change in the relationship between UV absorbance and DOC over 22 years at two upland moorland catchments in Scotland; despite increases in long-term DOC concentrations, their analysis suggests that the proportion of hydrophobic material declined. Erlandsson et al. [46] have reported on Swedish rivers where DOC and absorbance (420 nm) were measured between 1987 and 2004 and found that there was a significant increase in the absorbance/DOC ratio in 19 of the 21 rivers considered. They also found an increase in the COD/TOC ratio that corroborated that changes in the quality of the organic matter had occurred. Worrall and Burt [53] examined a seven-year record of daily coagulant/color records in a water treatment plant (Broken Scar, Scotland) and found that the DOC entering the water works was becoming increasingly difficult to remove by coagulation, suggesting that DOC was becoming more hydrophilic in this catchment. They also suggested that there was no reason to believe that the relationship between DOC and color had not shifted over the course of the study; although color showed no significant trend, it is possible that the DOC from the catchment was becoming less colored so that DOC could be increasing without a significant increase in color. Lepistö et al. [50] calculate the $\mathrm{C} / \mathrm{N}$ ratio (although not directly: OC by assuming a relationship between DOC and COD published in 1993 and organic nitrogen by difference between total and inorganic $\mathrm{N}$ ) and found that this $\mathrm{C} / \mathrm{N}$ ratio decreased during the study 
period (1962-2005), again pointing to a change in the type of organic matter. Temporal changes in the type of organic matter have also been recently shown in stored samples from lakes in the northeastern United States [101].

Finally, it should be added that in about $40 \%$ of the studies considered (Table 2), there is no information about the method used for OC quantification and that, rigorously, in these cases it is impossible to go further in the evaluation of the results obtained.

\subsubsection{Other Data Quality Issues}

Most of the available lake and river time series data are from national and local monitoring programs and it is often not possible to assess the quality of such data on the basis of the contents of the articles (e.g., reproducibility, use of certified reference materials, sampling procedures, etc.). With regard to trace elements, it was shown many years ago that much of the dissolved trace element work published by these programs was incorrect due to problems of contamination during sampling and analysis [102-104]. It is unknown whether this type of problem might have also affected OC measurements.

An additional aspect that needs to be considered is that data used in the trend studies are often monitoring data for regulatory purposes and that the fact that the objectives of this type of measurement differ from those of research-oriented studies is not without consequences. For instance, since the main concern of regulatory monitoring is simply to ascertain that some limit values are not exceeded, often not very sensitive techniques are used. The use of censored data (i.e., sets of data where some of the data are known to be "less than" some threshold) always introduces a bias in the magnitude of possible trends observed. The existence of this type of constraint has rarely, if ever, been mentioned in this field.

\subsubsection{Bias Towards Studying Systems "Where Something Happens"}

In addition to the well-known fact that citation practices confer on negative values an inherent quality of not spreading easily through the literature [7], authors rarely choose to study systems "where nothing happens" and often do not report results where the expected effects are not observed. Obviously, this has the automatic consequence of producing a bias in existing results and in the corresponding accepted belief. Although it is inherent to this type of problem that proving that it exists is well-nigh impossible, it is worth mentioning that it is highly probable that this behavior affects the subject considered here, leading to an overrepresentation of systems showing OC increasing trends.

\subsection{Data Treatment Aspects}

The second issue that needs to be considered is related to the way experimental data are treated. This information is set out in Table 2. Different aspects need to be discussed: data censoring, data transformation prior to their treatment and methods applied to detect temporal trends and to quantify them. 


\subsubsection{Data Censoring}

It is impossible to know when data has been censored because it is rarely mentioned explicitly in the studies considered (i.e., OC concentration detection limits and the number of values below these). Thus, it is impossible to assess the effect that data censoring might have in the conclusions reached. The only two cases found where explicit mention is made of "manual" censoring practices suggest a lack of understanding of the implications. A highly cited study [51] states: "sites with median concentrations of $<1 \mathrm{mg} / \mathrm{L}$ were excluded from our analysis" and this in order to restrict their analysis "to sites where DOC concentrations were sufficient to allow reliable quantification of trends". By doing this, the authors plainly ignored that values $\leq 1 \mathrm{mg} \mathrm{C} \mathrm{L}^{-1}$ are common in many systems. It also suggests a bias towards favoring systems with high DOC concentrations, which usually means northern humic-type ones. Since some authors [14,37,48,62,69] observed that trends are correlated with initial concentration levels, it is clear that, in practice, not considering low concentrations introduces a bias towards the measurement of higher trends. On the other end of the spectrum, de Wit et al. [52] wrote: "samples with exceptionally high TOC concentrations ( $\left.>18 \mathrm{mg} \mathrm{C} \mathrm{L}^{-1}\right)$ were excluded from the dataset" because "TOC in these samples had been modified by in-stream processes rather than being products of soil processes", reasoning difficult to understand when studying OC behavior in natural systems.

\subsubsection{Data Transformations}

When looking for trends and thus dealing with large data sets, different approaches are possible: use all data in the time series considered, sample a subset of the observations (i.e., plainly eliminate values), use mean values (e.g., monthly, yearly, etc.) calculated from measured ones. Using a subset or averaging is often done simply to ensure some regularity in the temporal distribution of the data in the series (sometimes required for the statistical methods applied). In the case of the time series considered here, the frequency of sampling is highly variable between studies and sometimes even within the same study (Table 1); very often original data are not used when applying data treatment methods but rather monthly or annual means are used instead. Since averaging is not an innocuous procedure [105], the exact procedure used, including sampling frequency and method of calculation of mean values, needs to be described in detail. Unfortunately, this information is very often omitted from the articles. In fact, this is one of the more difficult aspects to trace in the articles considered.

The way mean values are calculated in rivers influences the results obtained as shown by Eimers et al. [44]. These authors compared the effect of using volume-weighted and arithmetic means at seven headwater streams in Canada, obtaining different results. On average, annual measured DOC concentrations were $13 \%-34 \%$ higher than volume-weighted values and, although DOC increases were found in both cases, slopes were much larger in the measured data. Hruška et al. [36] also found differences in the magnitude of the trends observed (but not in the significance level) when using these two types of mean value calculation procedure.

The question merits some further discussion in the case of lakes. Depending on the size of the system (i.e., depth), many boreal and temperate lakes physically stratify in summer. Physical stratification drives chemical stratification and this needs to be taken into account when sampling. 
Again, in the studies considered here, information about how lake sampling has been performed is sometimes lacking (as is unfortunately the case in the studies covering a high number of systems like in $[18,51,71])$. When sampling methods are described, different strategies, not necessarily leading to comparable results, have been followed (Table 2). In general, only one value (obtained in very different ways, e.g., sampling at one fixed depth, integrated sampling, value averaging) per date is considered. This strategy simplifies calculations but, firstly, leads to non comparable results and, secondly, when OC is measured at only one point, it does not take into account that OC concentrations may evolve differently in surface waters than at depth and, when averaged or integrated, information is lost while possible existing trends might become less clear.

Data have sometimes been normalized using Z-scores [14,34,35,43,46]. Z-scores are calculated by subtracting from all values the mean over the period under study and dividing by the standard deviation. They have mostly been used for comparing systems with different levels of OC concentrations.

\subsubsection{Trend Detection and Quantification}

Since many water variables are not normally distributed, it is not generally appropriate to analyze them for temporal trends using parametric methods such as linear regression [106]. Accordingly, non-parametric methods have been largely used in the studies reviewed here. The non-parametric test for trends most frequently applied is the Kendall test (or Mann-Kendall, MK) [107,108]. The MK test compares every pair of values of the variable, and calculates the sign of the difference. The signs (indicating whether the second observation in each pair-wise comparison is higher, lower or equal than the first) for all pair-wise comparisons are summed and a Z-statistic calculated as the sum of signs divided by the standard deviation of the sum of signs. The statistical significance of any trend is indicated by the corresponding p-value. Unfortunately, the significance associated with the detection of a trend is not always given (Table 3).

There are many cases where concentrations in surface waters show strong seasonal patterns. This is often the case of OC. Ideally, seasonal variations must be removed in order to better discern any trend in the studied variable over time. The seasonal Kendall test (SMK) [106] accounts for seasonality by computing the MK test on each of the seasons separately and then combining the results. The SMK has been used in many OC trend studies (Table 2). The existence of seasonality can be tested by applying the Kruskal-Wallis statistic test but authors usually apply SMK without any previous test, presumably deducing seasonality visually or assuming that it probably exists. An alternative way of treating the effect of seasonality is applying trend tests (i.e., MK) to annual mean values. This approach has also been used. Although it eliminates seasonal effects and a part of the random variation of data, it has the drawback of reducing the information content.

The MK test does not estimate the magnitude of trends (slopes) but it has become usual to associate slopes calculated according to the method of Sen $[109,110]$ when a trend is detected. The method of Sen is a nonparametric method where the slope is approximated as the median value of all the pairwise slopes in the time series. However, a non negligible number of studies applied linear regression even after having used the MK method to find the existence of trends. 
The MK test is known to be well adapted to censored data (when only one censoring threshold exists; although note that the magnitude of the Sen slope is likely to be in error when using censored data), the presence of outliers and missing values. Limitations of the MK test are that there must be no serial correlation for the resulting p-values to be correct, and that data must be monotonic. A few of authors mention the use of modifications of the MK method that account for autocorrelation $[63,67,70,74]$; the most usual modification is the one proposed by Hirsch and Slack [111]. It is not excluded that others account for autocorrelation without mentioning it.

Monotonicity is potentially a serious problem in OC trend studies. In practice, monotonicity as such has rarely been statistically tested and data are generally assumed to be monotonic. However, cyclical patterns have been observed by some authors [34,35,46,56,65,77] either by looking at the graphical representation of the data or when applying smoothing methods such as the calculation of moving averages (also called running averages). Although not exempt from problems [105], calculation of moving averages provides a robust description of a data pattern and, although it has been seldom applied to OC data series, where it has been, cyclical patterns have appeared. Cyclical behavior has been observed in many parameters affected by climatic variations [112] and therefore is not astonishing that $\mathrm{OC}$ data show it, but it is worrying is that such behavior might have gone undetected in cases where monotonicity has been assumed. Obviously, for cyclicity to be apparent, long temporal series are needed.

It is common in hydrology to attempt to eliminate flow-related variability by adjusting water concentrations to flow (e.g., with LOWESS) and applying a trend test to the residuals. To our knowledge, this procedure has only been used by Burns et al. [55], after hyperbolic or log regression fit of the data. Other authors mention the use of the partial MK test with discharge or other parameters as covariates $[24,40]$.

Some authors estimate the magnitude of the trends by comparing initial and final values either in absolute terms or as a percentage. In principle, this should be avoided because, even if the mean of some initial and final years are used, the value remains very much dependent on initial and final conditions. Sometimes, even increases observed over a given period are extrapolated beyond it (e.g., Worrall et al. [30] found a 53.4\% increase over 8 years (1993-2000) but in the abstract talk about a $78 \%$ increase since 1970, "the period over which increase has been observed for the catchment as a whole").

\section{Conclusions}

Careful analysis of the 63 studies listed in Table 3 has revealed the existence of a number of problems both in the way results have been published (i.e., key information is missing from the articles, making it difficult to judge the reliability of the results; the degree of independence between published studies is fuzzy in some cases) and in the way OC analysis was performed (i.e., different, and not necessarily comparable, methods have been used along the years). In general, data treatment looks to be more problem free from the methodological point of view even if, sometimes, it is difficult to follow some methodological aspects such as, for instance, how authors pass from measured data to the numbers actually used in data treatment. This is not a trivial question because, as Stevenson and co-workers recently showed for temperature [105], different types of climatic patterns and anomalies 
are captured depending on which of various local and global methods are used. The failure to consider the possible existence of cyclicity, concomitant on the widespread assumption of data monotonicity, may also be a major flaw in OC studies that merits further consideration.

Can the initial question that motivated our study be answered in spite of these limitations? In brief, can we reasonably state that "there is a common trend of increasing concentrations of DOC in streams and lakes"? First, we think that it is clear to any reader that, because of the limited geographical coverage of the existing studies, if any general increasing OC trend did exist, our statement would need to be restricted to some northern zones of the North Hemisphere. Nearly no data exist for many areas of US and Europe temperate zones and none for the rest of the world.

That said, it would nevertheless be tempting to pool together observed trends and "play statistics" with them. However, this would have little sense for many reasons. First, in general: (i) studies cover very different temporal periods; (ii) the number of systems included in each individual study is very different, ranging from one intermittent stream [88] to a major comparison of 705 systems [66]; 33 of the published studies cover less than 10 systems and only 9 more than 100 . Curiously, if all trends were pooled together, the existence of four earlier studies [64,66,71,73] covering a huge number of lakes in Canada and Scandinavia (1324 in total), where about 90\% of the systems in each study showed no statistically significant trend, would probably give an overwhelming majority for the "non trend" category.

Secondly, how many of the 63 studies contain results that can be considered "usable"? A fast screening shows that not many. If studies that contain the same results published more than once by the same authors are eliminated from the initial 63 studies, 60 remain. Unfortunately, as explained above, there is no way of accounting for the interdependence of other published data. If we consider only studies which contain statistically significant results supported by a $\mathrm{p}$ value (irrespective of being increases, decreases or no trends), we are left with 37. And, if we fix the limit of significance at $p<0.05$, then only 34 of the initial 63 studies remain. It is worth mentioning here that judging this aspect is sometimes a bit tricky. For instance, in an important study covering 522 systems [51] there is no way of assessing the number of systems with significant trends (except trying to digitize some small figures appearing in the supporting information file) because the paper states than: "Upward slopes $(n=363)$ outnumbered downward slopes $(n=139)$, and $88 \%$ of significant trends $(p<0.05)$ were positive" without saying how many significant trends had been found. If of the remaining 34 studies, we consider only studies where OC has been measured as such (even leaving studies where correlations with surrogate parameters have been used for some periods of missing data), 27 studies remain. Finally, if we do not consider the studies where absolutely no information is given about the analytical methods used, we are left with no more than 11 . This last filter really reveals the limitations imposed by the lack of adequately reporting.

In conclusion, it is clear that OC concentrations have increased in some surface waters in the Northern Hemisphere since the 1990s. However, it cannot be proved that it is a general phenomenon because of the lack of data -temporal series- in many parts of the world and, as this study discusses, in the areas for which such series exist, the reporting and methodological problems in the published studies prevent so far reaching a conclusion about the existence of a general temporal behavior of OC concentrations. 


\section{Recommendations for Future Work}

Apart from the many different "technical" questions discussed along the different parts of this critical review, and that can be easily gleaned from the text, the main lesson to be learned from the situation described is the urgent need to improve the way both analytical and data treatment methods are reported.

Obviously, since this type of studies use already existing data, there is no room for improvement of their quality. The quality and types of data varies widely making it hard to analyze and harder still to use to detect temporal trends. However, it is possible, and extremely necessary, that raw data quality is carefully evaluated and that all details are given concerning analytical procedures used, data censoring, etc. Current publishing possibilities also make it feasible to make raw data easily available to all readers (e.g., through Supporting Information files, web-accessible files, etc.) facilitating appraisal and reuse.

Concerning data treatment, apart from the need to apply non-parametric methods -which are already widely used by this research community- there is no "best method" to recommend. However, methods used and any data pre-treatment applied should be, again, carefully detailed. Now that organic carbon concentrations have been determined for some systems for more than 30 years, systematic testing for cyclicity should be strongly encouraged in order to detect any possible climate-driven trend.

Finally, better citing practices will be welcome. Careful reading of the introduction of the 63 papers considered show a worrying repetition of the "accepted belief" that organic carbon concentration increases are widespread and a few papers are repeatedly cited. Citation "is not simply an impartial scholarly method for joining related published knowledge" [7]. Authors should probably be less guided by inertia when making the choice of which results to cite and which to ignore.

\section{Acknowledgement}

We thank the librarians of the old Center for Environmental Sciences (CCMA-CSIC) and of the National Museum of Natural History (MNCN-CSIC) for their help.

\section{Authors Contributions}

Montserrat Filella conceived the subject of the article and wrote it. Juan Carlos Rodríguez-Murillo contributed to gathering information and writing the article.
Abbreviations
A:
absorbance
COD:
chemical oxygen demand
DOC:
dissolved organic carbon
DOM:
dissolved organic matter
GC:
gas chromatography
HTC:
high temperature combustion
IR:
infrared
LOWESS:
locally weighted scatterplot smooth
LR:
lineal regression 


$\begin{array}{ll}\text { MK: } & \text { Mann-Kendall test } \\ \text { OC: } & \text { organic carbon } \\ \text { POC: } & \text { particulate organic carbon } \\ \text { POM: } & \text { particulate organic matter } \\ \text { SMK: } & \text { seasonal Mann-Kendall test } \\ \text { TOC: } & \text { total organic carbon } \\ \text { TON: } & \text { total organic nitrogen } \\ \text { UV: } & \text { ultraviolet } \\ \text { WCO: } & \text { wet carbon oxidation }\end{array}$

\section{Conflicts of Interest}

The authors declare no conflict of interest.

\section{References}

1. Randerson, J.T.; Chapin, III F.S.; Harden, J.W.; Neff, J.C.; Harmon, M.E. Net ecosystem production: A comprehensive measure of net carbon accumulation by ecosystems. Ecol. Appl. 2002, 12, 937-947.

2. Battin, T.J.; Luyssaert, S.; Kaplan, L.A.; Aufdenkampe, A.K.; Richter, A.; Tranvik, L.J. The boundless carbon cycle. Nat. Geosci. 2009, 2, 598-600.

3. Sobek, S.; Tranvik, L.J.; Prairie, Y.T.; Kortelainen, P.; Cole, J.J. Patterns and regulation of dissolved organic carbon: An analysis of 7,500 widely distributed lakes. Limnol. Oceanogr. 2007, 52, 1208-1219.

4. Porcal, P.; Koprivnjak, J.-F.; Molot, L.A.; Dillon, P.J. Humic substances-part 7: The biogeochemistry of dissolved organic carbon and its interactions with climate change. Environ. Sci. Pollut. Res. 2009, 16, 714-726.

5. Weyhenmeyer, G.A.; Karlsson, J. Nonlinear response of dissolved organic carbon concentrations in boreal lakes to increasing temperatures. Limnol. Oceanogr. 2009, 54, 2513-2519.

6. Sucker, C.; Krause, K. Increasing dissolved organic carbon concentrations in freshwaters: what is the actual driver? iForest 2010, 3, 106-108.

7. Greenberg, S.A. How citation distortions create unfounded authority: analysis of a citation network. Br. Med. J. 2009, 339, doi: 10.1136/bmj.b2680.

8. Monteith, D.T.; Stoddard, J.L.; Evans, C.D.; de Wit, H.A.; Forsius, M.; Høgåsen, T.; Wilander, A.; Skjelkvåle, B.L.; Jeffries, D.S.; Vuorenmaa, J.; et al. Dissolved organic carbon trends resulting from changes in atmospheric deposition chemistry. Nature 2007, 450, 537-541.

9. Skjelkvåle, B.L. The 15-Year Report: Assessment and Monitoring of Surface Waters in Europe and North America; Acidification and Recovery, Dynamic Modeling and Heavy Metals; ICP Waters Report 73/2003; Norwegian Institute for Water Research: Oslo, Norway, 2003.

10. Stoddard, J.L.; Kahl, J.S.; Deviney, F.A.; DeWalle, D.R.; Driscoll, C.T.; Herlihy, A.T.; Kellogg, J.H.; Murdoch, P.S.; Webb, J.R.; Webster, K.E. Response of Surface Water Chemistry to the Clean Air Act Amendments of 1990; Environmental Protection Agency, Office of Research and Development: Washington, DC, USA, 2003. 
11. Gruau, G.; Birgand, F.; Jarde, E.; Novince, E. Pollution des Captages d'Eau Brute de Bretagne par les Matières Organiques. Rapport de Synthèse Tableau de Bord de la Pollution Causes Possibles Recommandations, 2004. Available online: http://www.observatoire-eau-bretagne.fr/ Media/Documentation/Bibliographies/Pollution-des-captages-d-eau-brute-de-Bretagne-par-lesmatieres-organiques-Rapport-de-synthese.-Tableau-de-bord-de-la-pollution.-Causes-possibles.Recommandations (accessed on 24 March 2014).

12. Monchy, A.; Gruau, G. Pollution des Captages Superficiels d'Eau Brute des Pays de Loire par les Matières Organiques. Variabilité Spatiale Tendances Evolutives Causes Possibles et Recommandations. Comparaison avec le Cas de la Bretagne, 2010. Available online: http://halinsu.archives-ouvertes.fr/docs/00/57/81/09/PDF/PL02451.pdf (accessed on 23 March 2014).

13. Zobrist, J.; Sigg, L.; Schoenenberger, U. NADUD-Thematische Auswertung der Messresultate 1974 bis 1998; Schriftenreihe der EAWAG No. 18; Eidgenössische Anstalt für Wasserversorgung, Abwasserreinigung und Gewässerschutz (EAWAG): Dübendorf, Switzerland, 2004; Available online: http://library.eawag-empa.ch/schriftenreihe/ schriftenreihe_18.pdf (accessed on 9 May 2014).

14. Freeman, C.; Evans, C.D.; Monteith, D.T. Export of organic carbon from peat soils. Nature 2001, 412, 785.

15. Evans, C.D.; Chapman, P.J.; Clark, J.M.; Monteith, D.T.; Cresser, M.S. Alternative explanations for rising dissolved organic carbon export from organic soils. Glob. Chang. Biol. 2006, 12, 2044-2053.

16. Evans, C.D.; Monteith, D.T.; Cooper, D.M. Long-term increases in surface water dissolved organic carbon: Observations, possible causes and environmental impacts. Environ. Pollut. 2005, $137,55-71$.

17. Eikebrokk, B.; Vogt, R.D.; Liltved, H. NOM increase in Northern European source waters: Discussion of possible causes and impacts on coagulation/contact filtration processes. Water Sci. Technol. Water Supply 2004, 4, 47-54.

18. Skjelkvåle, B.L.; Stoddard, J.L.; Jeffries, D.S.; Tørseth, K.; Høgåsen, T.; Bowman, J.; Mannio, J.; Monteith, D.T.; Mosello, R.; Rogora, M.; et al. Regional scale evidence for improvements in surface water chemistry 1990-2001. Environ. Pollut. 2005, 137, 165-176.

19. Tranvik, L.J.; Downing, J.A.; Cotner, J.B.; Loiselle, S.A.; Striegl, R.G.; Ballatore, T.J.; Dillon, P.; Finlay, K.; Fortino, K.; Knoll, L.B.; et al. Lakes and reservoirs as regulators of carbon cycling and climate. Limnol. Oceanogr. 2009, 54, 2298-2314.

20. Amann, T.; Weiss, A.; Hartmann, J. Carbon dynamics in the freshwater part of the Elbe estuary, Germany: Implications of improving water quality. Estuar. Coast. Shelf Sci. 2012, 107, 112-121.

21. Couture, S.; Houle, D.; Gagnon, C. Increases of dissolved organic carbon in temperate and boreal lakes in Quebec, Canada. Environ. Sci. Pollut. Res. 2012, 19, 361-371.

22. Halliday, S.J.; Wade, A.J.; Skeffington, R.A.; Neal, C.; Reynolds, B.; Rowland, P.; Neal, M.; Norris, D. An analysis of long-term trends, seasonality and short-term dynamics in water quality data from Plynlimon, Wales. Sci. Total Environ. 2012, 434, 186-200.

23. Neal, C.; Robson, A.J.; Neal, M.; Reynolds, B. Dissolved organic carbon for upland acidic and acid sensitive catchments in mid-Wales. J. Hydrol. 2005, 304, 203-220. 
24. Kerr, J.G.; Eimers, M.C. Decreasing soil water $\mathrm{Ca}^{2+}$ reduces DOC adsorption in mineral soils: Implications for long-term DOC trends in an upland forested catchment in southern Ontario, Canada. Sci. Total Environ. 2012, 427-428, 298-307.

25. Eimers, M.C.; Dillon, P.J.; Schiff, S.L. Sulphate flux from an upland forested catchment in south-central Ontario. Water Air Soil Pollut. 2004, 152, 3-21.

26. Pärn, J.; Mander, Ü. Increased organic carbon concentrations in Estonian rivers in the period 1992-2007 as affected by deepening droughts. Biogeochemistry 2012, 108, 351-358.

27. Clair, T.A.; Dennis, I.F.; Vet, R. Water chemistry and dissolved organic carbon trends in lakes from Canada's Atlantic Provinces: no recovery from acidification measured after 25 years of lake monitoring. Can. J. Fish. Aquat. Sci. 2011, 68, 663-674.

28. Bertoni, R.; Callieri, C.; Corno, G.; Rasconi, S.; Caravati, E.; Contesini, M. Long-term trends of epilimnetic and hypolimnetic bacteria and organic carbon in a deep holo-oligomictic lake. Hydrobiologia 2010, 644, 279-287.

29. Clutterbuck, B.; Yallop, A.R. Land management as a factor controlling dissolved organic carbon release from upland peat soils 2: Changes in DOC productivity over four decades. Sci. Total Environ. 2010, 408, 6179-6191.

30. Worrall, F.; Burt, T.; Adamson, J. Can climate change explain increases in DOC flux from upland peat catchments? Sci. Total Environ. 2004, 326, 95-112.

31. Worrall, F.; Burt, T.P.; Adamson, J. Long-term changes in hydrological pathways in an upland peat catchment-recovery from severe drought? J. Hydrol. 2006, 321, 5-20.

32. Navrátil, T.; Norton, S.A.; Fernandez, I.J.; Nelson, S.J. Twenty-year inter-annual trends and seasonal variations in precipitation and stream water chemistry at the Bear Brook Watershed in Maine, USA. Environ. Monit. Assess. 2010, 171, 23-45.

33. van Kleef, H.H.; Brouwer, E.; Leuven, R.S.E.W.; van Dam, H.; de Vries-Brock, A.; van der Velde, G.; Esselink, H. Effects of reduced nitrogen and sulphur deposition on the water chemistry of moorland pools. Environ. Pollut. 2010, 158, 2679-2685.

34. Zhang, J.; Hudson, J.; Neal, R.; Sereda, J.; Clair, T.; Turner, M.; Jeffries, D.; Dillon, P.; Molot, L.; Somers, K.; et al. Long-term patterns of dissolved organic carbon in lakes across eastern Canada: Evidence of a pronounced climate effect. Limnol. Oceanogr. 2010, 55, 30-42.

35. Hudson, J.J.; Dillon, P.J.; Somers, K.M. Long-term patterns in dissolved organic carbon in boreal lakes: the role of incident radiation, precipitation, air temperature, southern oscillation and acid deposition. Hydrol. Earth Syst. Sci. 2003, 7, 390-398.

36. Hruška, J.; Krám, P.; McDowell, W.H.; Oulehle, F. Increased dissolved organic carbon (DOC) in central European streams is driven by reductions in ionic strength rather than climate change or decreasing acidity. Environ. Sci. Technol. 2009, 43, 4320-4326.

37. Oulehle, F.; Hruška, J. Rising trends of dissolved organic matter in drinking-water reservoirs as a result of recovery from acidification in the Ore Mts., Czech Republic. Environ. Pollut. 2009, 157, 3433-3439.

38. Sarkkola, S.; Koivusalo, H.; Laurén, A.; Kortelainen, P.; Mattsson, T.; Palviainen, M.; Piirainen, S.; Starr, M.; Finér, L. Trends in hydrometeorological conditions and stream water organic carbon in boreal forested catchments. Sci. Total Environ. 2009, 408, 92-101. 
39. Clair, T.A.; Dennis, I.F.; Vet, R.; Laudon, H. Long-term trends in catchment organic carbon and nitrogen exports from three acidified catchments in Nova Scotia, Canada. Biogeochemistry 2008, 87, 83-97.

40. Dawson, J.J.C.; Malcolm, I.A.; Middlemas, S.J.; Tetzlaff, D.; Soulsby, C. Is the composition of dissolved organic carbon changing in upland acidic streams? Environ. Sci. Technol. 2009, 43, 7748-7753.

41. Tetzlaff, D.; Malcolm, I.A.; Soulsby, C. Influence of forestry, environmental change and climatic variability on the hydrology, hydrochemistry and residence times of upland catchments. $J$. Hydrol. 2007, 346, 93-111.

42. McCartney, A.G.; Harriman, R.; Watt, A.W.; Moore, D.W.; Taylor, E.M.; Collen, P.; Keay, E.J. Long-term trends in $\mathrm{pH}$, aluminium and dissolved organic carbon in Scottish fresh waters; implications for brown trout (Salmo trutta) survival. Sci. Total Environ. 2003, 310, 133-141.

43. Eimers, M.C.; Buttle, J.; Watmough, S.A. Influence of seasonal changes in runoff and extreme events on dissolved organic carbon trends in wetland- and upland-draining systems. Can. J. Fish. Aquat. Sci. 2008, 65, 796-808.

44. Eimers, M.C.; Watmough, S.A.; Buttle, J.M. Long-term trends in dissolved organic carbon concentration: a cautionary note. Biogeochemistry 2008, 87, 71-81.

45. Eimers, M.C.; Watmough, S.A.; Buttle, J.M.; Dillon, P.J. Examination of the potential relationship between droughts, sulphate and dissolved organic carbon at a wetland-draining stream. Glob. Chang. Biol. 2008, 14, 938-948.

46. Erlandsson, M.; Buffam, I.; Fölster, J.; Laudon, H.; Temnerud, J.; Weyhenmeyer, G.A.; Bishop, K. Thirty-five years of synchrony in the organic matter concentrations of Swedish rivers explained by variation in flow and sulphate. Glob. Chang. Biol. 2008, 14, 1191-1198.

47. Futter, M.N.; Starr, M.; Forsius, M.; Holmberg, M. Modelling the effects of climate on long-term patterns of dissolved organic carbon concentrations in the surface waters of a boreal catchment. Hydrol. Earth Syst. Sci. 2008, 12, 437-447.

48. Vuorenmaa, J.; Forsius, M.; Mannio, J. Increasing trends of total organic carbon concentrations in small forest lakes in Finland from 1987 to 2003. Sci. Total Environ. 2006, 365, 47-65.

49. Keller, W.; Paterson, A.M.; Somers, K.M.; Dillon, P.J.; Heneberry, J.; Ford, A. Relationships between dissolved organic carbon concentrations, weather, and acidification in small Boreal Shield lakes. Can. J. Fish. Aquat. Sci. 2008, 65, 786-795.

50. Lepistö, A.; Kortelainen, P.; Mattsson, T. Increased organic C and N leaching in a northern boreal river basin in Finland. Glob. Biogeochem. Cycles 2008, 22, GB3029.

51. Monteith, D.T.; Evans, C.D. United Kingdom Acid Waters Monitoring Network 10 Year Report. Analysis and Interpretation of Results, April 1988-March 1998. London, 2000. Available online: http://awmn.defra.gov.uk/resources/interpreports/10yearInterpRpt.pdf (accessed on 23 March 2014).

52. De Wit, H.A.; Mulder, J.; Hindar, A.; Hole, L. Long-term increase in dissolved organic carbon in streamwaters in Norway is response to reduced acid deposition. Environ. Sci. Technol. 2007, 41, 7706-7713.

53. Worrall, F.; Burt, T.P. Trends in DOC concentration in Great Britain. J. Hydrol. 2007, 346, 81-92. 
54. Worrall, F.; Harriman, R.; Evans, C.D.; Watts, C.D.; Adamson, J.; Neal, C.; Tipping, E.; Burt, T.; Grieve, I.; Monteith, D.; et al. Trends in dissolved organic carbon in UK rivers and lakes. Biogeochemistry 2004, 70, 369-402.

55. Burns, D.A.; McHale, M.R.; Driscoll, C.T.; Roy, K.M. Response of surface water chemistry to reduced levels of acid precipitation: comparison of trends in two regions of New York, USA. Hydrol. Process. 2006, 20, 1611-1627.

56. Dillon, P.J.; Molot, L.A. Long-term trends in catchment export and lake retention of dissolved organic carbon, dissolved organic nitrogen, total iron, and total phosphorous: The Dorset, Ontario, study, 1978-1998. J. Geophys. Res. 2005, 110, G01002.

57. Findlay, S.E.G. Increased carbon transport in the Hudson River: Unexpected consequence of nitrogen deposition? Front. Ecol. Environ. 2005, 3, 133-137.

58. Arvola, L.; Räike, A.; Kortelainen, P.; Järvinen, M. The effect of climate and landuse on TOC concentrations and loads in Finnish rivers. Boreal Environ. Res. 2004, 9, 381-387.

59. Hongve, D.; Riise, G.; Kristiansen, J.F. Increased colour and organic acid concentrations in Norwegian forest lakes and drinking water-a result of increased precipitation? Aquat. Sci. 2004, 66, 231-238.

60. Worrall, F.; Burt, T.P. Time series analysis of long-term river dissolved organic carbon records. Hydrol. Process. 2004, 18, 893-911.

61. Worrall, F.; Burt, T.; Shedden, R. Long term records of riverine dissolved organic matter. Biogeochemistry 2003, 64, 165-178.

62. Driscoll, C.T.; Driscoll, K.M.; Roy, K.M.; Mitchell, M.J. Chemical response of lakes in the Adirondack region of New York to declines in acidic deposition. Environ. Sci. Technol. 2003, 37, 2036-2042.

63. Driscoll, C.T.; Van Dreason, R. Seasonal and long-term temporal patterns in the chemistry of Adirondack Lakes. Water Air Soil Pollut. 1993, 67, 319-344.

64. Forsius, M.; Vuorenmaa, J.; Mannio, J.; Syri, S. Recovery from acidification of Finnish lakes: regional patterns and relations to emission reduction policy. Sci. Total Environ. 2003, 310, 121-132.

65. Hejzlar, J.; Dubrovský, M.; Buchtele, J.; Růžička, M. The apparent and potential effects of climate change on the inferred concentration of dissolved organic matter in a temperate stream (the Malše River, South Bohemia). Sci. Total Environ. 2003, 310, 143-152.

66. Jeffries, D.S.; Clair, T.A.; Couture, S.; Dillon, P.J.; Dupont, J.; Keller, W.; McNicol, D.K.; Turner, M.A.; Vet, R.; Weeber, R. Assessing the recovery of lakes in southeastern Canada from the effects of acidic deposition. Ambio 2003, 32, 176-182.

67. Keller, W.; Heneberry, J.H.; Dixit, S.S. Decreased acid deposition and the chemical recovery of Killarney, Ontario, lakes. Ambio 2003, 32, 183-189.

68. Gunn, J.N.; Snucins, E.; Yan, N.D.; Arts, M.T. Use of water clarity to monitor the effects of climate change and other stressors on oligotrophic lakes. Environ. Monit. Assess. 2001, 67, 69-88.

69. Harriman, R.; Watt, A.W.; Christie, A.E.G.; Collen, P.; Moore, D.W.; McCartney, A.G.; Taylor, E.M.; Watson, J. Interpretation of trends in acidic deposition and surface water chemistry in Scotland during the past three decades. Hydrol. Earth Syst. Sci. 2001, 5, 407-420.

70. Skjelkvåle, B.L.; Stoddard, J.; Andersen, T. Trends in surface water acidification in Europe and North America (1989-1998). Water Air Soil Pollut. 2001, 130, 787-792. 
71. Skjelkvåle, B.L.; Mannio, J.; Wilander, A.; Andersen, T. Recovery from acidification of lakes in Finland, Norway and Sweden 1990-1999. Hydrol. Earth Syst. Sci. 2001, 5, 327-333.

72. Apsite, E.; Klavins, M. Assessment of the changes of COD and color in rivers of Latvia during the last twenty years. Environ. Int. 1998, 24, 637-643.

73. Mallory, M.L.; McNicol, D.K.; Cluis, D.A.; Laberge, C. Chemical trends and status of small lakes near Sudbury, Ontario, 1983-1995: evidence of continued chemical recovery. Can. J. Fish. Aquat. Sci. 1998, 55, 63-75.

74. Bouchard, A. Recent lake acidification and recovery trends in Southern Quebec, Canada. Water Air Soil Pollut. 1997, 94, 225-245.

75. Schindler, D.W.; Curtis, P.J.; Bayley, S.E.; Parker, B.R.; Beaty, K.G.; Stainton, M.P. Climate-induced changes in the dissolved organic carbon budgets of boreal lakes. Biogeochemistry 1997, 36, 9-28.

76. Forsberg, C. Will an increased greenhouse impact in Fennoscandia give rise to more humic and coloured lakes? Hydrobiologia 1992, 229, 51-58.

77. Andersson, T.; Nilsson, Å.; Jansson, M. Coloured substances in Swedish lakes and rivers-Temporal variation and regulating factors. Lect. Notes Earth Sci. 1991, 33, 243-253.

78. Forsberg, C.; Petersen, R.C. A darkening of Swedish lakes due to increased humus inputs during the last 15 years. Verh. Int. Ver. Limnol. 1990, 24, 289-292.

79. Naden, P.S.; McDonald, A.T. Statistical modelling of water colour in the Uplands: The Upper Nidd catchment 1979-1987. Environ. Pollut. 1989, 60, 141-163.

80. Watts, C.D.; Naden, P.S.; Machell, J.; Banks, J. Long term variation in water colour from Yorkshire catchments. Sci. Total Environ. 2001, 278, 57-72.

81. Yallop, A.R.; Clutterbuck, B. Land management as a factor controlling dissolved organic carbon release from upland peat soils 1: Spatial variation in DOC productivity. Sci. Total Environ. 2009, 407, 3803-3813.

82. Köhler, S.; Hruška, J.; Bishop, K. Influence of organic acid site density on pH modeling of Swedish lakes. Can. J. Fish Aquat. Sci. 1999, 56, 1461-1470.

83. Koprivnjak, J.-F.; Blanchette, J.G.; Bourbonniere, R.A.; Clair, T.A.; Heyes, A.; Lum, K.R.; McCrea, R.; Moore, T.R. The underestimation of concentrations of dissolved organic carbon in freshwaters. Water Res. 1995, 29, 91-94.

84. Ivarsson, H.; Jansson, M. Regional variation of dissolved organic-matter in running waters in central northern Sweden. Hydrobiologia 1994, 286, 37-51.

85. Gadmar, T.C.; Vogt, R.D.; Osterhus, B. The merits of the high temperature combustion method for determining the amount of natural organic carbon in surface freshwater samples. Int. J. Environ. Anal. Chem. 2002, 82, 451-461.

86. Mattsson, T.; Kortelainen, P.; Räike, A. Export of DOM from boreal catchments: impacts of land use cover and climate. Biogeochemistry 2005, 76, 373-394.

87. Kortelainen, P. Content of total organic-carbon in Finnish lakes and its relationship to catchment characteristics. Can. J. Fish. Aquat. Sci. 1993, 50, 1477-1483.

88. Dawson, J.J.C.; Soulsby, C.; Tetzlaff, D.; Hrachowitz, M.; Dunn, S.M.; Malcolm, I.A. Influence of hydrology and seasonality on DOC exports from three contrasting upland catchments. Biogeochemistry 2008, 90, 93-113. 
89. Futter, M.N.; de Wit, H.A. Testing seasonal and long-term controls of streamwater DOC using empirical and process-based models. Sci. Total Environ. 2008, 407, 698-707.

90. Jonsson, A.; Meili, M.; Bergström, A.-K.; Jansson, M. Whole-lake mineralization of allochthonous organic carbon in a large humic lake (Örträsket, N. Sweden). Limnol. Oceanogr. 2001, 46, 1691-700.

91. Algesten, G.; Sobek, S.; Bergström, A.-K.; Ågren, A.; Tranvik, L.J.; Jansson, M. Role of lakes for organic carbon cycling in the boreal zone. Glob. Chang. Biol. 2004, 10, 141-147.

92. Sharp, J.H. Analytical methods for total DOM pools. In Biogeochemistry of Marine Dissolved Organic Matter; Hansell, D.A., Carlson, C.A., Eds. Academic Press: San Diego, CA, USA, 2002; pp. 35-58.

93. Suzuki, Y. On the measurement of DOC and DON in seawater. Mar. Chem. 1993, 42, 287-288.

94. Dafner, E.V.; Wangersky, P.J. A brief overview of modern directions in marine DOC studies. Part I.-Methodological aspects. J. Environ. Monit. 2002, 4, 48-54.

95. Ogawa, H.; Tanoue, E. Dissolved organic matter in oceanic waters. J. Oceanogr. 2003, 59, 129-147.

96. Benner, R.; Hedges, J.I. A test of the accuracy of freshwater DOC measurements by high-temperature catalytic oxidation and UV-promoted persulfate oxidation. Mar. Chem. 1993, $41,161-165$.

97. Kaplan, L.A. Comparison of high-temperature and persulfate oxidation methods. Limnol. Oceanogr. 1992, 37, 1119-1125.

98. Wangersky, P.J. Dissolved organic carbon methods: A critical review. Mar. Chem. 1993, 41, 61-74.

99. Buffle, J. Complexation Reactions in Aquatic Systems. An Analytical Approach. Ellis Horwood: Chichester, UK, 1988.

100. Filella, M. Freshwaters: Which NOM matters? Environ. Chem. Lett. 2009, 7, 21-35.

101. SanClements, M.D.; Oelsner, G.P.; McKnight, D.M.; Stoddard, J.L.; Nelson, S.J. New insights into the source of decadal increases of dissolved organic matter in acid-sensitive lakes of the Northeastern United States. Environ. Sci. Technol. 2012, 46, 3212-3219.

102. Shiller, A.M.; Boyle, E.A. Variability of dissolved trace metals in the Mississippi River. Geochim. Cosmochim. Acta 1987, 51, 3273-3277.

103. Windom, H.L.; Byrd, J.T.; Smith, R.G.; Huan, F. Inadequacy of NASQAN data for assessing metal trends in the Nation's rivers. Environ. Sci. Technol. 1991, 25, 1137-1142.

104. Taylor, H.E.; Shiller, A.M. Mississippi River methods comparison study: Implications for water quality monitoring of dissolved trace elements. Environ. Sci. Technol. 1995, 29, 1313-1317.

105. Stevenson, K.; Alessa, L.; Altaweel, M.; Kliskey, A.D.; Krieger, K.E. Minding our methods: How choice of time series, reference dates, and statistical approach can influence the representation of temperature change. Environ. Sci. Technol. 2012, 46, 7435-7441.

106. Hirsch, R.M.; Slack, J.R.; Smith, R.A. Techniques of trend analysis for monthly water quality analysis. Water Resour. Res. 1982, 18, 107-121.

107. Mann, H.B. Non-parametric test against trend. Econometrica 1945, 13, 245-249.

108. Kendall, M.G. Rank Correlation Methods, 4th ed.; Charles Griffin: London, UK, 1975.

109. Theil, H. A rank-invariant method of linear and polynomial regression analysis. Adv. Stud. Theor. Appl. Econom. 1992, 23, 345-381. 
110. Sen, P.K. Estimates of the regression coefficient based on Kendall's tau. J. Am. Stat. Assoc. 1968, 63, 1379-1389.

111. Hirsch, R.M.; Slack, J.R. A nonparametric trend test for seasonal data with serial dependence. Water Resour. Res. 1984, 20, 727-732.

112. Peixoto, J.P.; Oort, A.H. Interannual and interdecadal variability in the climate system. In Physics of Climate; American Institute of Physics: New York, NY, USA, 1992; Chapter 16, pp. 412-449.

(C) 2014 by the authors; licensee MDPI, Basel, Switzerland. This article is an open access article distributed under the terms and conditions of the Creative Commons Attribution license (http://creativecommons.org/licenses/by/3.0/). 